\title{
SEARCH FOR SINGLE TOP PRODUCTION WITH THE D DETECTOR AT THE FERMILAB TEVATRON COLLIDER
}

\author{
Name: Jeffrey E. McDonald \\ Department: Department of Physics \\ Major Professor: Harrison B. Prosper \\ Degree: Doctor of Philosophy \\ Term Degree Awarded: Summer, 1999
}

We attempt to identify a single top signal in the muon + jets data collected using the $\mathrm{D} \varnothing$ detector at the Fermilab Tevatron at a center-of-mass energy of $\sqrt{s}=1.8$ TeV from 1992 - 96. The data corresponds to an integrated luminosity of $103.7 \mathrm{pb}^{-1}$. While resolving a signal has proved impossible, we are able to place an upper limit on the production cross section for single top events using these data. The cross section for production of single top via $95 \% p \bar{p} \rightarrow$ tb and $p \bar{p} \rightarrow$ qtb is less than $78 \mathrm{pb}$ at the $95 \%$ confidence level based on muon + jets data only. 
THE FLORIDA STATE UNIVERSITY

COLLEGE OF ARTS AND SCIENCES

SEARCH FOR SINGLE TOP PRODUCTION WITH THE D DETECTOR AT THE FERMILAB TEVATRON COLLIDER

By

JEFFREY E. MCDONALD

\begin{abstract}
A Dissertation submitted to the Department of Physics in partial fulfillment of the requirements for the degree of Doctor of Philosophy
\end{abstract}

Degree Awarded:

Summer Semester, 1999 
The members of the Committee approve the dissertation of Jeffrey E. McDonald defended on May 5, 1999.

Harrison B. Prosper

Professor Directing Dissertation

R. C. Lacher

Outside Committee Member

Horst D. Wahl

Committee Member

Stephan L. Linn

Committee Member

Howard A. Baer

Committee Member

Lawrence C. Dennis

Committee Member 
I dedicate this dissertation to my dad and mom, without whose inspiration and support I would have surely failed. 


\section{ACKNOWLEDGEMENTS}

I begin by thanking all those who have kept me sane and looking forward over the past four and a half years. Those "behind the scenes" people don't often get the respect and the recognition they deserve. My family and friends have served as a guiding light from the very beginning. You contribute so much, but ask for so little. Thank you from the top to the bottom of my heart for all of your love and support.

When you stop to take stock of things learned, you cannot help but admire the people who gave you the necessary skills for the accomplishments that you've made. The high energy physics faculty at Florida State have contributed very much to my success. I especially thank Horst Wahl, Stephan Linn, Vasken Hagopian, Howard Baer, and J. F. Owens for the skills, support and encouragement that made this work possible.

Working with a collaboration of more than 450 physicists, it is often difficult to know whom to thank for contributions to a work. Therefore, I recognize the entire collaboration, not just those physicists who get the limelight, but also the engineers, the technicians, and the support staff. Great people make for a great detector!

A number of close personal colleagues contributed much to my success. First among these are my advisors. Harrison Prosper and Pushpa Bhat have been with me

through the trials and the tribulations. Their guidance has allowed me to venture to new heights. I wish also to recognize their unerring faith in me, and encouragement, to seek new paths, despite the risk.

Were it not for the Single Top Group, led valiantly by Ann Heinson, my task would have been insurmountable. I thank them for all of their work, long hours and dedication to the single top analysis. 
Inside the collaboration, I'd like to recognize the support from John Hobbs, Krish Gounder and the entire top analysis group, especially, Prof. Tom "Hey, don't do that!" Ferbel.

Finally, many thanks are due to the other students resident at DØ. The FSU students Dan Karmgard, Russell Gilmartin, Brian Connolly, and Guoliang Wang have been especially civil office mates. Terry Heuring, late of FSU, has been a lantern in the dark on many a night. To these and the always unmentioned, I'm thankful for the long discussions and reality checks.

I cannot end without a word of thanks to my most treasured friends. Among those, Andrew Green, Harpreet Singh and Tina Hebert have all been there for me in times of joy and less happy times. Thanks especially to Elizabeth for making my life balanced and joyful. Thanks for being such great friends and colleagues; your commitments will not soon be forgotten. 


\section{CONTENTS}

List of Tables $\ldots \ldots \ldots \ldots \ldots \ldots \ldots \ldots \ldots \ldots \ldots \ldots \ldots \ldots \ldots \ldots \ldots \ldots$ viii

List of Figures $\ldots \ldots \ldots \ldots \ldots \ldots \ldots \ldots \ldots \ldots \ldots \ldots \ldots \ldots \ldots \ldots \ldots \ldots \ldots$

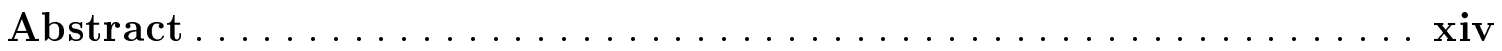

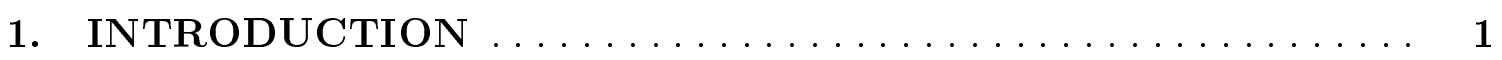

1.1 The Most Fundamental Branch of Science . . . . . . . . . . . . 1

1.2 Particle Physics . . . . . . . . . . . . . . . . . . . 1

2. THE STANDARD MODEL $\ldots \ldots \ldots \ldots \ldots \ldots \ldots \ldots \ldots \ldots$

2.1 The Cast of the Standard Model $\ldots \ldots \ldots \ldots \ldots \ldots \ldots \ldots . \quad 7$

2.2 Standard Model Conclusions . . . . . . . . . . . . . . . 18

3. SINGLE TOP THEORY $\ldots \ldots \ldots \ldots \ldots \ldots \ldots \ldots \ldots \ldots$

3.1 Top Discovered! . . . . . . . . . . . . . . . . . . 20

3.2 Single Top Quark Production Cross Sections............... 21

3.3 Modeling Single Top Quark Production. . . . . . . . . . . . 24

3.4 Single Top Quark Measurements ................... 34

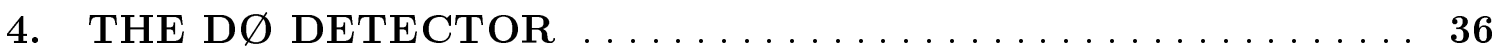

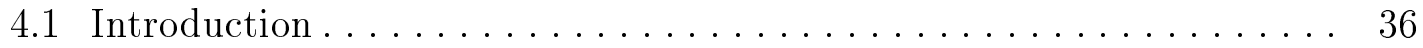

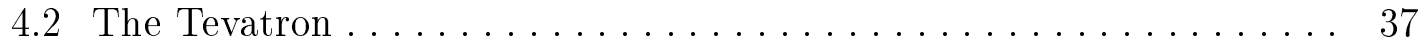

4.3 Coordinate System....................... 40

4.4 Central Detectors........................... 41

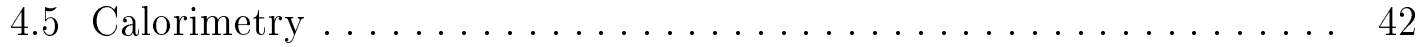

4.6 Muon Detection ........................... 48

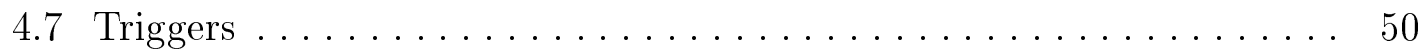

4.8 Data Acquisition ............................ 52

4.9 Modeling the Detector Response . . . . . . . . . . . . . 53

5. PARTICLE RECONSTRUCTION AND IDENTIFICATION . . 54

5.1 Particle Reconstruction . . . . . . . . . . . . . . . . . . . 54

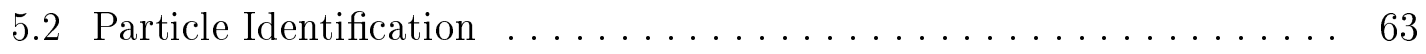

5.2 .1 Muon ID . . . . . . . . . . . . . . . . . . . . 63 


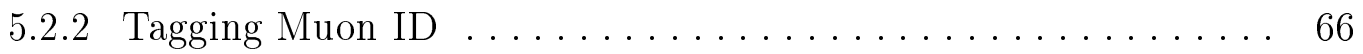

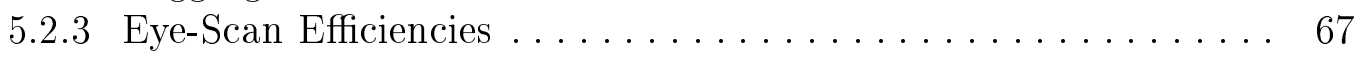

5.2.4 Jets and Missing Transverse Energy .............. 68

6. MUON+JETS WITH $\mu$-TAG ANALYSIS $\ldots \ldots \ldots \ldots \ldots \ldots 70$

6.1 Analysis Synopsis........................... 70

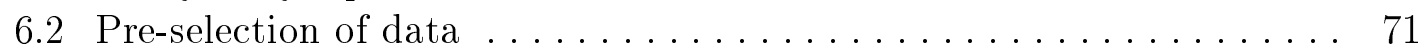

6.3 The Selection Requirements.................. 74

6.4 The Heavy Flavor (QCD) Tagrate Function . . . . . . . . . . . 75

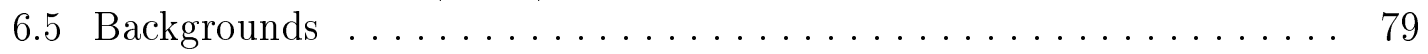

6.5.1 QCD Multi-Jet Background to the $\mu$-tag Analysis . . . . . . 79

$6.5 .2 \mathrm{~W}+$ jet background $\ldots \ldots \ldots \ldots \ldots \ldots \ldots \ldots \ldots \ldots \ldots \ldots \ldots \ldots$

$6.5 .3 t \bar{t}$ Backgrounds $\ldots \ldots \ldots \ldots \ldots \ldots \ldots \ldots \ldots \ldots \ldots .67$

6.5.4 Total Background to the single top $\mu+$ jets $/ \mu$ channels . . . . . 98

6.6 Single Top Acceptances . . . . . . . . . . . . . . . . . . . 100

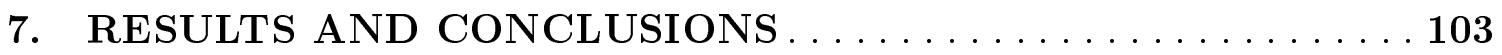

7.1 Cross Section Limits for Single Top . . . . . . . . . . . . . 103

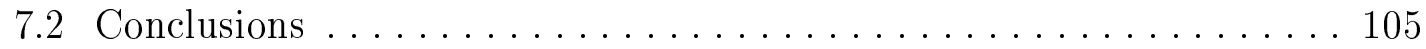

\section{APPENDICES}

A. USEFUL DEFINITIONS $\ldots \ldots \ldots \ldots \ldots \ldots \ldots \ldots \ldots \ldots \ldots$

B. LUMINOSITY, TRIGGERS AND VETO CONDITIONS $\ldots \ldots 112$

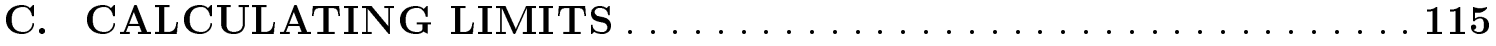

D. MUON + JETS/UNTAGGED ANALYSIS $\ldots \ldots \ldots \ldots \ldots \ldots 118$

E. SINGLE TOP SENSITIVITY IN RUN II $\ldots \ldots \ldots \ldots \ldots \ldots 124$

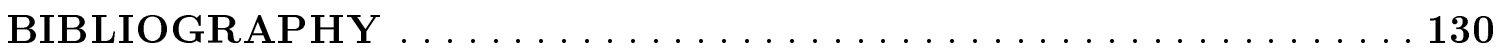

BIOGRAPHICAL SKETCH $\ldots \ldots \ldots \ldots \ldots \ldots \ldots \ldots \ldots \ldots$ 


\section{LIST OF TABLES}

2.1 The fundamental particles of the Standard Model. . . . . . . . . . 8

3.1 A typical event from PYTHIA for single top quark t-channel production at the Fermilab Tevatron. The first column is the particle index number (I). The second and third columns are the particle name (particle) and the index of the "mother" particle (orig). The remaining columns are the particle's momenta (p_x,p_y,p_z) in GeV/c, energy (E) in GeV and

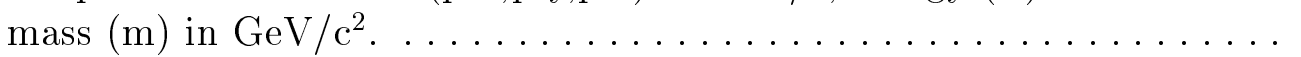

3.2 A typical event from PYTHIA for single top quark s-channel production at the Fermilab Tevatron. The first column is the particle index number (I). The second and third columns are the particle name (particle) and the index of the "mother" particle (orig). The remaining columns are the particle's momenta (p_x,p_y,p_z) in GeV/c, energy (E) in $\mathrm{GeV}$ and

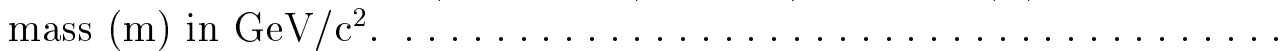

3.3 Predicted event yields for single top and the primary backgrounds. The yield is for an integrated luminosity of $100 \mathrm{pb}^{-1} \ldots \ldots \ldots \ldots \ldots$

5.1 Muon efficiencies for the definitions used in this analysis. The muon represented here are from the decay of the W. A good muon candidate passes both the Muon Track Badness cuts and calorimeter verification. PMUO is the bank that contains information about the reconstructed muon. . . . . . . . . . . . . . . . . . . . . . . . . .

5.2 Muon efficiencies for detection of a tagging muon from a b-jet. Both $\mathrm{CF}$ and EF muons are represented here. Recall that no events with EF muons are used before run $89000 . \ldots \ldots \ldots \ldots \ldots \ldots \ldots \ldots \ldots \ldots \ldots$

6.1 Events pre-selected for Run I. Only the Run IB background sample was used, the number of pre-selected events in this sample was 334,906 . . 73

6.2 Number of data events surviving selection cuts. . . . . . . . . . . 75

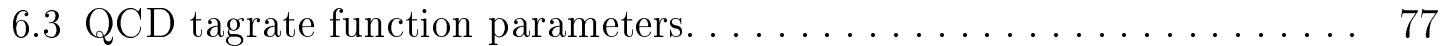

6.4 Fake rates as a function of $\mathbb{E}_{T}$ for CF muons only. The result for EF muons are similar. The cuts used here do not reject $\mathrm{Z}+$ jets events or

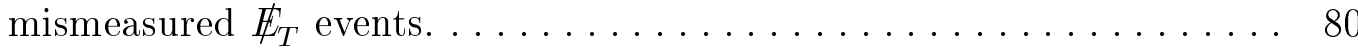


6.5 QCD multi-jet calculation for $\mu+$ jets $/ \mu$-tag analysis, for CF muons only. The numbers of events listed are for untagged QCD mult-jet

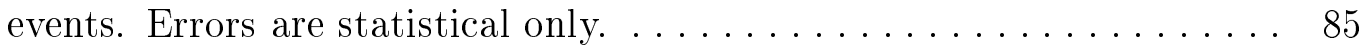

6.6 QCD multi-jet calculation for $\mu+$ jets/ $\mu$-tag analysis, for EF muons only. The numbers of events listed are for untagged QCD multi-jet events. Errors are statistical only. . . . . . . . . . . .

6.7 QCD multi-jet calculation for $\mu+$ jets $/ \mu$-tag analysis. Results are shown for both $\mathrm{CF}$ and EF muons. The total QCD multi-jet background is $2.2 \pm 0.54$ events. Errors in the table are statistical only. . . . . . . . 86

6.8 Summary of data events and kinematic quantities. nj ${ }^{\text {Good }}$ is the number of jets with $E_{T}>15$. and $|\eta|<2.5$. This data sample includes all of Run I. A asterisk indicates that the event is a $t \bar{t}$ candidate from previous

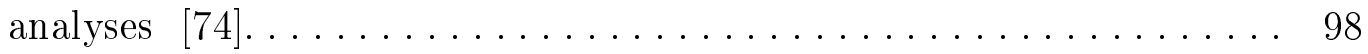

6.9 Total number of events expected in muon + jets/tagged analysis. This is for Run IB only. . . . . . . . . . . . . . . . . 100

6.10 Acceptances for single top MC without requiring the muon tag. The trigger efficiencies are for events with a tagging muon for a MU_JET_XXX filter. The MC geometric acceptances and branching fractions for tagging muons vary from $7.5 \%$ to $16.7 \%$. . . . . . . . 102

6.11 The single top efficiencies for finding the tagging muon. The $\epsilon_{\text {soft }}^{\mu}$ includes all efficiencies except the eye-scan efficiency. All single top events have two $b$-quarks. . . . . . . . . . . . . . . . . . . 102

7.1 Single top identification efficiencies for the single top to muon + jets channel with a tagging muon. The efficiencies include trigger, muon identification efficiencies and the efficiency for finding a tagging muon. . 104

7.2 Single top acceptances for the muon + jets channel with a tagging muon and the acceptance for single top to any final state. The acceptances are calculated using Eq. 6.7. The two processes are weighted by the cross sections and combined, see Eq. 7.1. . . . . . . . . . . . . 104

7.3 Cross section limits for the $\mu+$ jets $/ \mu$ channels. . . . . . . . . . 105

B.1 The luminosity calculation results for the $t \bar{t}$ mu+jets data stream. The luminosity error is $5.4 \%$. The luminosity quoted for Run IA has been subjected to a $5 \%$ loss in luminosity because of reprocessing

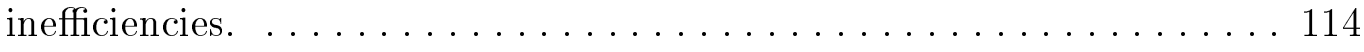

D.1 QCD multi-jet calculation for muon+jets analysis. Both $\mathrm{CF}$ and $\mathrm{EF}$ muons are allowed in this calculation. Here, only the trigger, good muon cuts and jet cuts are applied. Errors are statistical only. . . . . 120 
D.2 QCD multi-jet calculation for muon+jets analysis. Only CF muons are allowed in this calculation. Errors are statistical only. . . . . . . 120

D.3 QCD multi-jet calculation for muon+jets analysis. Only EF muons are allowed in this calculation. Errors are statistical only. . . . . . . 121

E.1 Acceptances for single top and backgrounds for leptonic and hadronic channels. The single top t-channel has been separated into two distinct channels. In fact $q b \rightarrow t q$ is included in the process $g q \rightarrow t q b$, but at the time of the study it was thought that there were reasons to distinguish the processes. The all-hadronic channel is not well studied and the QCD background is expected to dominate all other physics processes. . 128

E.2 Events yields for single top events for signal and backgrounds to any decay channel, to leptonic decay channels and all-hadronic decay channels. The yield is calculated per $\mathrm{fb}^{-1}$ of luminosity. . . . . . . . 129 


\section{LIST OF FIGURES}

2.1 A diagram showing the elements of a physical process with initial state hadrons, an underlying process and final state hadronization. . . . . . . 13

3.1 The Feynman diagram for inclusive single top production as shown in

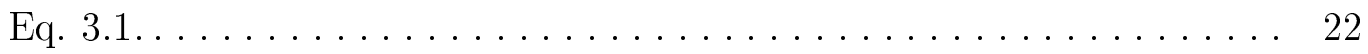

3.2 The leading order diagrams for single top quark production via W-gluon fusion. . . . . . . . . . . . . . . . . . 23

3.3 The leading order diagrams for single top quark production via the $W^{*}$

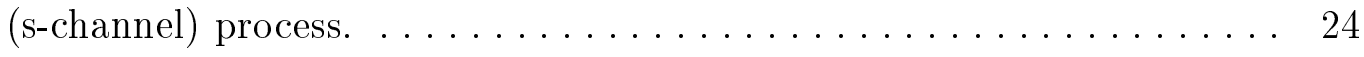

3.4 Some kinematic quantities for the single top quark t-channel processes

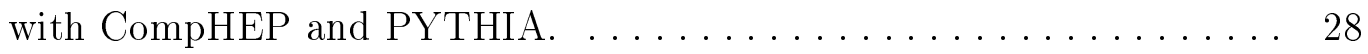

3.5 Some kinematic quantities for the single top quark t-channel processes with CompHEP and PYTHIA. Here, cuts have been applied to remove the low- $p_{T}$ spectator quarks (below $15 \mathrm{GeV} / \mathrm{c}$ ) and low- $p_{T}$ final state light quarks (below $10 \mathrm{GeV} / \mathrm{c}$ ). The agreement is much better between

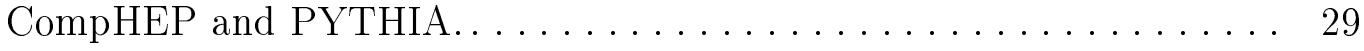

3.6 Some kinematic quantities for the single top quark s-channel processes

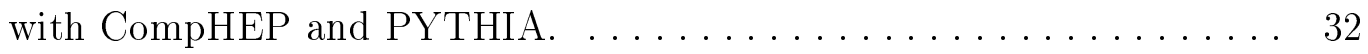

4.1 The Fermilab Tevatron proton/anti-proton synchrotron. . . . . . . . . 38

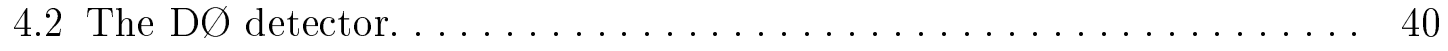

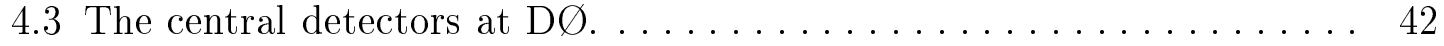

4.4 The forward drift chambers for the DØdetector. The forward drift chambers consists of a two modules for theta measurement between a module for phi measurement. . . . . . . . . . . . . . . . . 43

4.5 A cutaway view of the $\mathrm{D} \varnothing$ Liquid Argon Calorimeter........... . 45

4.6 A side view of one quarter of the $\mathrm{D} \varnothing$ detector, showing segmentation and tower definitions. Lines from the center extend along constant pseudorapidities...................... 46

4.7 A side view of DØdetector, showing the entire detector muon system. . 49 
$6.1 \eta$ distributions of the soft muon for a data background sample and a $t \bar{t}$ sample of events. The $t \bar{t}$ sample was chosen because of its enrichment

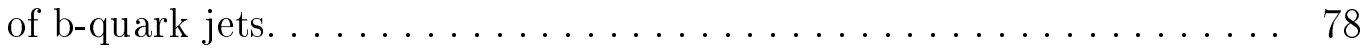

6.2 Isolated muon fake rates as a function of Missing Transverse Energy. . 81

6.3 An isolated QCD event in which a jet is lost. The assumption here is that if we had a perfect detector, there would be no instrumental QCD events where a muon is not inside the cone of a jet. However, the physical process in which muons escape the cone of a jet would contribute to a QCD multi-jet background. In the low $\mathbb{E}_{T}$ region, there is little $W$ contamination and the events are assumed to be mostly QCD. 88

6.4 A non-isolated QCD event. The muon meets all the requirements of an isolated one, but fails the isolation requirement $\Delta R>0.5 . \ldots \ldots \ldots$

6.5 A non-isolated QCD event passing all analysis cuts, except muon isolation. The number of fake events is determined from the number of events of this type multiplied by the fake rate (and convolved with the

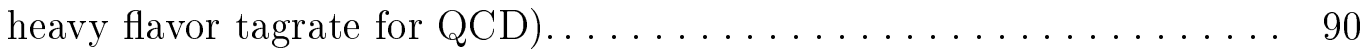

6.6 Tagged data and untagged data using tagrate7. The tagged data are shown as open squares and the untagged are shown as filled circles. The event selection has been relaxed for a factor of three additional statistics. The cuts applied were 2 or more jets and an isolated (untagged) or tagging (tagged) muon. . . . . . . . . . . . . 91

6.7 Tagged data and untagged data using tagrate7. The tagged data are shown as open squares and the untagged are shown as filled circles. The events shown passed a tight set of cuts, which did not require a tight isolated muon. . . . . . . . . . . . . . . . . . . . . . . . . 92

6.8 The $t \bar{t}$ background kinematic distributions for several variables. The events are subject to selection cuts (see Section 6.3) . . . . . . . . . 93

6.9 The $\mathrm{W}+$ jets background kinematic distributions for several variables. The events are subject to loose selection cuts, those in Section 6.3, without the tagging muon requirement. The model is Vecbos+Isajet. . . 94

6.10 The QCD fake isolated background for the $\mu+$ jets/tagged analysis. The events are weighted by the heavy flavor tagrate function. . . . . . . . 95

6.11 The single top t-channel kinematic distributions for several variables. The events are subject to selection cuts (see Section 6.3). The model is CompHEP + PYTHIA. . . . . . . . . . . . . . . . . . 96 
6.12 The single top s-channel kinematic distributions for several variables. The events are subject to selection cuts (see Section 6.3). The model is CompHEP+PYTHIA. . . . . . . . . . . . . . . . . 97

6.13 The signal and background distributions shown as functions of kinematic variables. The signal and background have been normalized to their calculated values, see Table 6.9 and Fig. $6.14 \ldots \ldots \ldots \ldots \ldots .99$

6.14 The distribution of backgrounds between QCD, $W+$ jets and Top backgrounds. . . . . . . . . . . . . . . . . . . . . . . 101 


\begin{abstract}
We attempt to identify a single top signal in the muon + jets data collected using the DØ detector at the Fermilab Tevatron at a center-of-mass energy of $\sqrt{s}=1.8$ TeV from 1992 - 96. The data corresponds to an integrated luminosity of $103.7 \mathrm{pb}^{-1}$. While resolving a signal has proved impossible, we are able to place an upper limit on the production cross section for single top events using these data. The cross section for production of single top via $95 \% p \bar{p} \rightarrow$ tb and $p \bar{p} \rightarrow$ qtb is less than $78 \mathrm{pb}$ at the $95 \%$ confidence level based on muon + jets data only.
\end{abstract}

xiv 


\section{CHAPTER 1}

\section{INTRODUCTION}

\subsection{The Most Fundamental Branch of Science}

When I was old enough to begin to question the world around me, I found that there were so many questions and so few answers that I knew. While I have become privy to a small set of answers that I sought, I've discovered there are far, far more to ask.

My journey has taken me far and the rewards have been greater than any other path I could have chosen. When I was a senior high school student, my thirst for knowledge was quenched by the elegant logic and the answers that I found in physics.

Physics concerns itself with the entire world. An understanding and mathematical description of the laws that govern all motion and all physical phenomena is the ultimate goal of physics. Physics is the most fundamental of the natural sciences.

\subsection{Particle Physics}

In the 1920s, a new branch of physics sprouted roots that would grow into High Energy or Particle Physics. Following the late 1800s declaration that nearly all physics problems had been solved with the theories of Newton for motion and Maxwell's for electromagnetism, the physics community was presented with the first manifestations of the naiveté of this point of view. 
The Planck theory of blackbody radiation began the revolution known as quantum mechanics. Planck and other physicists found that if one modeled a black body cavity with harmonic oscillators, the classical theories of Newton and Maxwell predicted a non-physical result. The result was known as the ultraviolet catastrophe. Planck found, however, that if the energy of harmonic oscillators were allowed to take on a discrete set of values rather than a continuous one, the resulting theory successfully described experiment. Planck had quantized the harmonic oscillator. Several years later, when experiments began probing atoms, physicists discovered again that they had to quantize the model.

With the Schrödinger equation, physicists found that they could successfully model atomic interactions. The Schrödinger equation and quantum mechanics in general, made predictions which were counter-intuitive and led some to proclaim "God does not play dice" [1]. Particle physics entered a new era when relativity was added to the quantum mechanical description. A shortcoming of the Schrödinger description was its inability to conform to relativistic invariance.

Just a few years after the development of the Schrödinger equation, Dirac proposed the theory that would eventually blossom into quantum electrodynamics. A startling prediction was made: the existence of an anti-electron. The prediction of anti-matter would be difficult for the physics community to readily accept, until its discovery several years after the theoretical prediction by Dirac. Ultimately, the Dirac theory accurately predicted the phenomenology of electrons and photons.

One of the least understood physical processes during the 1930s was the $\beta$-decay of the neutron. In this process, it was observed that a neutron would spontaneously decay into a proton with the emission of an electron. In 1933, Enrico Fermi proposed a physical description for the process [2]. However, there was a problem: it appeared that energy was not conserved. A new weakly interacting particle was proposed, 
called the neutrino, whose purpose was to carry away the energy and thereby preserve the law of conservation of energy. Fermi used the theory of electromagnetism as a guide for describing the $\beta$-decay of the neutron. This description proved very useful and predicted a new type of force which was weaker than the electromagnetic force. Although Fermi's description was useful, at higher energies the Fermi theory predicted unphysical results. Quantum field theory would eventually be used to describe a viable theory which had better high energy behavior.

Out of the Dirac theory, a theory of quantum fields evolved to explain ever more complicated quantum systems. The first such theory developed by Feynman, Tomonaga and Schwinger is Quantum Electrodynamics or QED for short. A key advance was the introduction by Feynman of a diagrammatic way to represent physical processes and a systematic recipe for calculating observables.

With QED, one could picture the photon as the particle which "mediated" the electromagnetic force. In the 1960s a theory analogous to QED was sought to describe $\beta$-decay in the hope of improving the Fermi theory. However, it was known that the weak force was very short ranged and therefore, the particles that mediated the forces must be massive. Earlier, Fermi proposed the "contact interaction" as an effective description of the weak interactions, but the theory predicted non-physical results at high energies. At the time of this realization, it was not known how to mathematically describe in a consistent way a massive field with a short range force.

The theory of beta decay eventually developed into the theory of weak interactions. These interactions describe the weak nuclear forces and will be discussed in Chapter 2.

High energy physics or particle physics grew out of the quantum theory of matter as a separate branch of physics whose purpose is to study the most elementary particles of matter and their interactions. The principal method of study is to cause 
particles to collide at the highest possible energies using accelerators and to observe the particle interactions with special detectors.

With higher energies, smaller and smaller length scales are revealed. The ability to look inside an object and search for structure is an important part of high energy physics. To be able to describe nature with a few simple laws is the ultimate goal of particle physics.

During the 1960s, experimental results guided theoretical developments of the quark model. The origins of the quark model were found in hadron spectroscopy of deep-inelastic lepton-nucleon scattering. These experiments were interpreted as indications that hadrons were not fundamental particles but were composed of more fundamental point-like particles call quarks.

As the energy of the interactions increased, greater resolution was possible and access to higher mass quarks or quark-quark bound states was possible. To date, six quarks have been found, with the top quark being found most recently in 1995 at the Fermilab Tevatron [3-4]. The discovery of the high mass quarks would not have been possible without the facilities provided by laboratories like Fermilab.

This thesis deals with the physics of single top quark interactions. The single top interactions are interesting because they probe the interactions of the top quark which was discovered only four years ago. In addition to expanding our knowledge of the top quark, this study will broaden our understanding of the quark model and how the top quark fits into the model. The models and phenomenology of single top processes will be described in Chapter 3 .

At Fermilab, there are two general-purpose experiments at the Tevatron collider: CDF and D $\varnothing$. These experiments are very large and complex. The D $\varnothing$ detector is a state of the art detector with over a million channels of information. The detector will be described in Chapter 4 . 
It is not possible to directly measure quantities that are physically significant. Instead, we have a large array of digitized signals which must be converted into the physically meaningful quantities. This is called event reconstruction and will be discussed in Chapter 5.

The theoretical clean room of physics analysis becomes smeared and muddied by the detector's finite resolution and imperfections. In order to observe a single top quark event one must first find such an event. The process of searching for such events with a real detector will be discussed in Chapter 6 . The results of this analysis and conclusions will be reported in Chapter 7 . 


\section{CHAPTER 2}

\section{THE STANDARD MODEL}

Our goal in physics is to see things simply, to understand a great many complicated phenomena in a unified way, in terms of a few simple principles. - Steven Weinberg

\section{Introduction}

Our observations of nature often do not allow us to immediately grasp all that is apparent. As Feynman put it, we seek the "wheels within wheels." It is this drive to understand nature that leads us to develop theories of nature. We wish not only to observe nature, but to understand it. How do the electrons, neutrinos, nucleons interact with each other and what is the nature of these particles? How can we understand the physics of elementary particles using simple mathematical principles?

A fundamental principle of contemporary high energy physics is that matter and energy can be described with theories called gauge field theories. When quantized these gauge theories lead to the idea of particle exchange as the mechanism of particle interactions. For example, all electromagnetic interactions can be described as the exchange of photons between charged particles. What happens when we observe a

more complicated process such as the $\beta$-decay of a neutron? Can we describe such a process using the idea of particle exchange? 
The next level of complexity are the nuclear forces that hold the nucleus together. The nuclear force is difficult to describe mathematically because of its complex nature and because of its strength. Can we understand the nuclear force as arising from an exchange of a particle?

Physics can answer many questions regarding how the universe is put together. It is important to remember that our keys to understanding the universe begin by understanding the simplest principles and then building more complex theories. Thus began the development of the Standard Model of Particle Physics. The Standard Model is motivated and developed in a number of texts [5-8].

\subsection{The Cast of the Standard Model}

The prevailing model of particle physics, the Standard Model, has been tested for over twenty years without serious difficulties. To date, the Standard Model has been able to accommodate all experimental results with the exception of recent solar atmospheric neutrino data.

The Standard Model particles, quarks $(q)$ and leptons $(l)$, have spin 1/2 and are point-like particles to a level of $10^{-18} \mathrm{~m}$. There are three families of quarks and leptons, constituting a total of six "flavors" of each. The quarks interact via an exchange of spin 1 gauge bosons: eight massless gluons, the massless photon and the massive gauge bosons, $\mathrm{W}^{ \pm}$and $\mathrm{Z}^{0}$. The leptons interact with only the photon and the massive gauge bosons. The particle content of the Standard Model is shown in Table 2.1.

\section{Symmetry Groups of the Standard Model}

A group describes symmetries which preserve physically significant quantities. The symmetry of a group can assist in visualizing and calculating physical observ- 
Table 2.1. The fundamental particles of the Standard Model.

\begin{tabular}{|c|c|c|c|}
\hline Particle & Charge & $\operatorname{Mass}\left(\mathrm{GeV} / \mathrm{c}^{2}\right)$ & Interactions \\
\hline \multicolumn{4}{|c|}{ Leptons } \\
\hline Electron (e) & -1 & $5.11 \times 10^{-4}$ & EM, Weak \\
\hline Electron Neutrino $\left(\nu_{e}\right)$ & 0 & $<5.1 \times 10^{-9}$ & Weak \\
\hline Muon $(\mu)$ & -1 & 0.1057 & EM, Weak \\
\hline Muon Neutrino $\left(\nu_{\mu}\right)$ & -1 & $<2.7 \times 10^{-4}$ & Weak \\
\hline $\operatorname{Tau}(\tau)$ & -1 & 1.771 & EM, Weak \\
\hline Tau Neutrino $\left(\nu_{\tau}\right)$ & -1 & $<0.031$ & Weak \\
\hline \multicolumn{4}{|c|}{ Quarks } \\
\hline $\mathrm{Up}(u)$ & $+2 / 3$ & $\approx 0.005$ & EM, Weak, Color \\
\hline $\operatorname{Down}(d)$ & $-1 / 3$ & $\approx 0.010$ & EM, Weak, Color \\
\hline $\operatorname{Charm}(c)$ & $2 / 3$ & $\approx 1.30$ & EM, Weak, Color \\
\hline Strange $(s)$ & $-1 / 3$ & $\approx 0.20$ & EM, Weak, Color \\
\hline $\operatorname{Top}(t)$ & $2 / 3$ & $174.3 \pm 5.1$ & EM, Weak, Color \\
\hline $\operatorname{Bottom}(b)$ & $-1 / 3$ & $\approx 4.3$ & EM, Weak, Color \\
\hline \multicolumn{4}{|c|}{ Bosons } \\
\hline Photon $(\gamma)$ & 0 & 0 & EM \\
\hline W-Boson $\left(W^{ \pm}\right)$ & \pm 1 & 80.22 & Weak (Charged) \\
\hline Z-Boson $\left(Z^{0}\right)$ & 0 & 91.187 & Weak (Neutral Current) \\
\hline Gluon $(G)$ & 0 & 0 & Color \\
\hline
\end{tabular}


ables. As an example, the conservation of angular momentum is a consequence of invariance under rotations in space. This invariance under rotations is manifest in the $\mathrm{SU}(2)$ symmetry group.

The structure of the Standard Model is invariant under the action of the direct product of three groups:

$$
S U(3)_{C} \times S U(2)_{L} \times U(1)_{Y}
$$

The $S U(3)_{C}$ color group represents symmetries of the strong interactions of the Standard Model. Each quark field is labelled by an index that can assume three different values called, arbitrarily, red, green and blue. The structure of the Standard Model is invariant with respect to the rotation in the space spanned by these indices. Each generator of the group is associated with one massless, spin 1 gluon which carries color charge. There are eight gluons and thus, eight generators of the " $S U(3)_{C}$ rotation". The quarks interact via the gluon field to form bound states of hadrons. The coupling constant between the quarks and gluons $\left(\alpha_{s}\right)$ is of order one at energies less than one $\mathrm{GeV}$ but evolves to much lower values at high energy.

The $S U(2)_{L} \times U(1)_{Y}$ group unifies electromagnetism and weak interactions, collectively known as the electro-weak interactions. The $S U(2)_{L}$ describes weak isospin and acts on left-handed doublets, for example, $\left(\begin{array}{c}v_{e} \\ e\end{array}\right)$. The $U(1)_{Y}$ is associated with weak hypercharge ${ }^{1}$ interactions. The symmetry of the electroweak interaction is "spontaneously" broken by the Higgs mechanism. The Higgs mechanism is the process by which a local gauge theory describing massless particles can be made to describe massive particles while preserving the renormalizability of the theory. The key idea is the introduction of a doublet of a complex scalar fields

\footnotetext{
${ }^{1}$ Hypercharge is a function of the third component of isospin and the electric charge.
} 
(the Higgs field) to break the symmetry of the theory and give mass to the particles in the theory. With the addition of the doublet of scalar fields, one gains four degrees of freedom. Three of the degrees of freedom are used to generate the three massive gauge bosons $W^{+}, W^{-}$and $Z^{0}$. In order to gain mass, each of the massive gauge bosons gain a degree of freedom which is manifest by a longitudinal polarization in addition to the transverse polarizations. The photon $(\gamma)$ remains massless and retains its two degrees of freedom. There is a single degree of freedom left from the doublet of scalar fields: the Higgs boson. The Higgs boson is the only vital part of the Standard Model which remains to be discovered.

\section{Quantum Chromodynamics}

Quantum chromodynamics is the theory of the strong interactions between quarks. This theory describes the structure of hadrons. A baryon is a hadron which is composed of three quarks, $B=\left(q_{a}, q_{b}, q_{c}\right)$. A meson is a composite state of two quarks, $M=\left(q_{a}, \bar{q}_{b}\right)$. The quarks are spin $\frac{1}{2}$ particles and are point-like. The baryons have half-integer spin and the mesons have integral spin.

An early puzzle in the theory of baryon structure was the apparent symmetry of some of the baryonic states. The wave function of some baryons appeared to be symmetric under the interchange of particle indices. This violated Fermi-Dirac statistics. Spin- $\frac{1}{2}$ particles obey Fermi-Dirac statistics, which states that the wave function should be anti-symmetric under the interchange of particle indices. The fact that the baryons were apparently symmetric under the exchange of indices led to the proposal of a new quantum number for the quarks, called color. With the extra degrees of freedom the baryonic states could be made anti-symmetric under exchange of quark indices. 
The $S U(3)_{C}$ group is associated with the exact invariance of the strong interactions under the exchange of color indices. The $S U(3)_{C}$ theory predicts that physical states are color singlets. Thus, ordinary hadrons can only be combinations of two and three quark states, the mesons and baryons. Since no free quarks are observed in nature, the coupling constant of quantum chromodynamics (QCD) is constrained to predict that there are no free quark states.

The prediction that no free quarks exists is found to be experimentally true. In order to keep free quarks from appearing, one requires a strong dynamical mechanism for binding quarks together. QCD has eight colored vector fields, called gluons. The eight fields are necessary to respect the exact color symmetries. QCD predicts a self-coupling of the gluon because of its non-Abelian nature. In a theory based on an Abelian group, there are no interactions among the fields associated with the generators of the group.

Most predictions of QCD are calculable only at high energies where the coupling constant is small enough to allow for the perturbative description of the interactions. However, a property of both QCD and QED are divergences which appear in the calculation of observables. Fortunately, there is a scheme for handling the divergences called "renormalization," where divergences are swept into the definitions of the parameters such as the coupling constant and mass. A renormalizable theory is one in which only a finite number of parameters is needed to account for all of the divergences in the theory. When a theory is renormalized, the predictions are physical and the divergences disappear.

The coupling constant of QCD is denoted $\alpha_{s}$. After renormalization the coupling constant becomes interaction energy $\left(q^{2}\right)$ dependent, where $q^{2}$ is the momentum scale of the interaction; $\alpha_{s}\left(q^{2}\right)$ can be represented as 


$$
\alpha_{s}\left(q^{2}\right)=\frac{\alpha_{s}\left(\mu_{0}^{2}\right)}{1+\frac{\left(11 N_{c}-2 N_{f}\right)}{12 \pi} \alpha_{s}\left(\mu_{0}^{2}\right) \ln \left(\frac{-q^{2}}{\mu_{0}^{2}}\right)} .
$$

In Eq. 2.2, $\alpha_{s}\left(\mu_{0}^{2}\right)$ is the renormalized coupling at an arbitrary momentum scale $\mu_{0}$; $N_{c}$ is the number of colors and $N_{f}$ is the number of active flavors. There are three colors and as many as six flavors in QCD. The number of active flavors increases with momentum transfer. Consequently, the coupling constant decreases when additional flavors become active. This is called asymptotic freedom. For this reason, physicists are able to justify using perturbation theory at high energies for the calculation of the observables of QCD. In perturbation theory, an observable is expanded in powers of the coupling constant. In order to perform a reliable perturbative calculation, the coupling constant must be much less than unity.

A crucial element of QCD calculations is the factorization theorem, Eq. 2.3. The factorization theorem states that an observable, for example a cross section, can be factorized into a calculable perturbative part and a non-perturbative, currently non-calculable part. In the calculation of a cross section the non-perturbative part is represented by the parton distribution functions. A parton distribution function represents the probability density of finding a parton of a given flavor with a given fraction of the hadron's momentum. It is tempting to interpret the parton distribution functions as describing the structure of hadrons. However, one should resist the temptation in view of the factorization theorem which tells us that the dividing line between PDFs and the perturbative part is arbitrary.

In Eq. 2.3, the non-pertubative factors are represented by $F_{i}\left(x_{1}, M\right)$ and $F_{j}\left(x_{2}, M\right)$. The functions $F_{i}\left(x_{1}, M\right)$ and $F_{j}\left(x_{2}, M\right)$ are the parton distribution functions (PDFs). These functions are parameterized in terms of the parton momentum fractions $x_{1}$ and $x_{2}$ and in terms of any other Standard Model parameters represented 


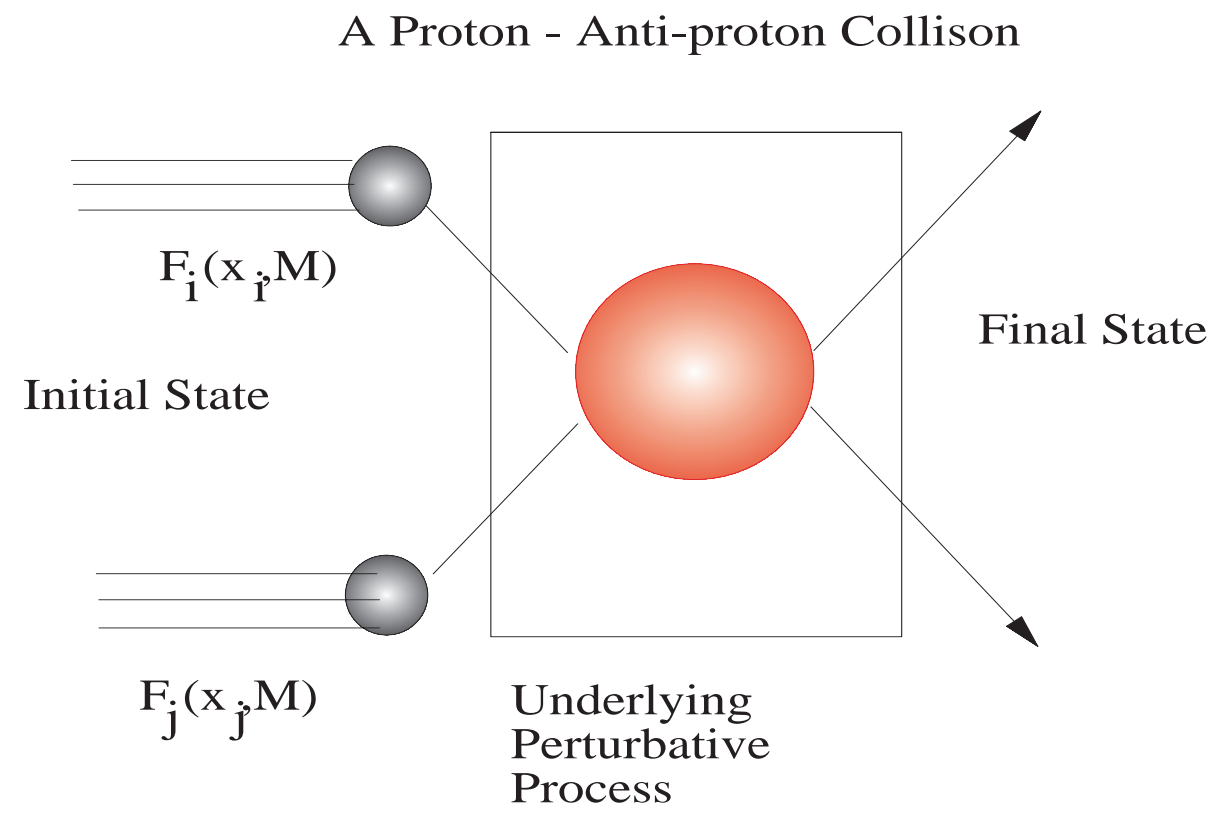

Figure 2.1. A diagram showing the elements of a physical process with initial state hadrons, an underlying process and final state hadronization.

by $\mathrm{M}$. The perturbative part of the observable is represented by $\hat{\sigma}_{i j}$. This equation is shown pictorially in Fig. 2.1.

$$
d \sigma^{p \bar{p} \rightarrow X}=\sum_{i, j} \int d x_{1} d x_{2} F_{i}\left(x_{1}, M\right) F_{j}\left(x_{2}, M\right) \hat{\sigma}_{i j}
$$

In practice, there are a number of parton distribution functions available for use in calculating these processes. Similarly, there are a number of generators of the perturbative process as well. Typically, the Coordinated Theoretical-Experimental Project on QCD (CTEQ) [9] provides parton distribution functions to be used with the CompHEP [36-37] or PYTHIA [38] programs for describing the perturbative (hard-scattering) processes. The details of CompHEP and PYTHIA will be discussed in Chapter 3. Since the final state of a QCD process contains no free quarks, it is necessary to model also the hadronization of the final state partons into colorless 
mesons or baryons. PYTHIA includes a model of hadronization however, CompHEP does not.

When a process is calculated, there must be a systematic method for removing the divergences of the theory. This is the renormalization scheme. The most commonly used scheme for removing the divergences of the theory is the minimal subtraction scheme $(\overline{\mathrm{MS}})[10]$.

\section{The Electro-Weak Interactions}

A triumph of the Standard Model was the unification of the electromagnetic and weak forces based on the group $S U(2)_{L} \times U(1)_{Y}$ [11-13]. Experimentally, it was noted that the weak current is of the form $V-A$, that is, a vector minus an axial vector. Any fermion field can be decomposed into left-handed and right-handed components. In Eq. 2.4, the fermion field is decomposed into left and right components. In Eq. 2.4, $\gamma_{5}$ (an axial vector $)=\gamma_{1} \gamma_{2} \gamma_{3} \gamma_{4}$, where $\gamma_{i}$ are the Dirac matrices [6]. Only left-handed components contribute to $V-A$ interactions.

$$
\begin{aligned}
\Psi(x) & =\Psi_{L}(x)+\Psi_{R}(x) \\
\Psi_{L}(x) & =\left(1-\gamma_{5}\right) \Psi(x) \\
\Psi_{R}(x) & =\left(1+\gamma_{5}\right) \Psi(x)
\end{aligned}
$$

The electro-weak theory is developed by requiring that the Lagrangian be invariant under local $S U(2)$ gauge transformations, Eq. 2.5. The isopin doublet, more generally called a spinor, is formed by the left-handed components of a lepton or quark family; denoted by $\left(\begin{array}{c}v_{e L}(x) \\ e_{L}(x)\end{array}\right)$ for the electron family.

$$
\left(\begin{array}{c}
v_{e L}(x) \\
e_{L}(x)
\end{array}\right) \rightarrow U(x)\left(\begin{array}{c}
v_{e L}(x) \\
e_{L}(x)
\end{array}\right)
$$


To make the theory gauge invariant, new fields had to be introduced into the theory. The $S U(2)$ gauge invariance gives rise to three new fields, $W_{\lambda}^{a}(x)$. If in addition to $S U(2)$ invariance the theory is required to preserve $U(1)$ gauge invariance, an additional field is introduced, $B_{\lambda}(x)$. The $U(1)$ invariance introduces two additional degrees of freedom as two arbitrary phases, one for the left-handed doublet and one for the right-handed singlet spinor components, called weak hypercharge.

The Lagrangian density for the $S U(2)$ and $U(1)$ gauge invariant theory is given by

$$
\mathcal{L}(x)=-\frac{1}{2} \operatorname{Tr}\left(W_{\lambda \rho}(x) W^{\lambda \rho}(x)\right)-\frac{1}{4} B_{\lambda \rho}(x) B^{\lambda \rho}(x)+\bar{\psi} i \gamma^{\lambda} D_{\lambda} \psi(x) .
$$

In Eq. 2.6, $W_{\lambda \rho}(x)$ represents the fields added to preserve $S U(2)$ gauge invariance, $\operatorname{Tr}$ is the matrix trace operation, $B^{\lambda \rho}(x)$ preserves $U(1)$ gauge invariance and $D_{\lambda}$ is a covariant derivative ${ }^{2}$.

When the terms of Eq. 2.6 are expanded out, if left-handed fermions are chosen to have hypercharge $-\frac{1}{2}$ and right-handed fermions chosen to have an hypercharge of 0 , the right-handed terms of the expression vanish. This is required for a $V-A$ theory.

The $Z$ and $A$ fields are introduced as linear combinations, the $B_{\lambda}$ field and the third component of the $W_{\lambda}$ field as follows

$$
\begin{aligned}
& Z_{\lambda}=\cos \theta_{W} W_{\lambda}^{3}-\sin \theta_{W} B_{\lambda} \\
& A_{\lambda}=\sin \theta_{W} W_{\lambda}^{3}+\cos \theta_{W} B_{\lambda} .
\end{aligned}
$$

The hypercharge, a free parameter, is chosen as above and then the "unphysical" system rotated into a "physical" one. In Eq. 2.7, fields $Z_{\lambda}$ and $A_{\lambda}$ are recognized as the $Z^{0}$ boson and photon fields, respectively.

\footnotetext{
${ }^{2} \mathrm{~A}$ covariant derivative preserves the gauge invariance of the theory.
} 


\section{The Higgs Mechanism}

As discussed in Section 2.1, the Higgs mechanism is invoked to give masses to the fermions and to the massive gauge bosons. The implementation of the Higgs mechanism for non-Abelian gauge theories is developed in a number of texts, see for example, [5-7]. The development of the Higgs mechanism for $U(1)$ Abelian gauge theories begins by adding a scalar potential $V(\Psi)$ to the Lagrangian density,

$$
\begin{aligned}
\mathcal{L} & =-\frac{1}{4} F_{\mu \nu} F^{\mu \nu}+D_{\mu} \Psi^{\dagger} D^{\mu} \Psi-V(\Psi), \\
V(\Psi) & =-\mu^{2} \Psi^{\dagger} \Psi+h\left(\Psi^{\dagger} \Psi\right)^{2} .
\end{aligned}
$$

In Eq. 2.8, $D_{\mu}=\partial_{\mu}+i e A_{\mu}$ and $F_{\mu \nu}$ represents the field strength of the field $A_{\mu}$.

If $\mu^{2}>0$, the field $\Psi$ will acquire a vacuum expectation value and the local symmetry of $U(1)$ will be spontaneously broken. The symmetry is spontaneously broken because the Lagrangian density, through the minimum of the potential, has acquired a particular vacuum expectation value. That is, the vacuum state fails to exhibit the symmetry of the theory.

The potential will have a minimum at $\Psi_{0}=\left(\frac{\mu^{2}}{h}\right)^{1 / 2}$. The field is expanded about the vacuum state and the potential is re-written in terms of the expansion, Eq. 2.9,

$$
\begin{aligned}
\Psi(x) & =\Psi_{0}+\frac{1}{\sqrt{2}}\left(\Psi_{1}(x)+i \Psi_{2}(x)\right), \\
V(\Psi) & =-\frac{1}{2 h} \mu^{4}+\frac{1}{2} \cdot 2 \mu^{2} \Psi_{1}^{2}+\text { Higher Order Terms. }
\end{aligned}
$$

One observes that the field $\Psi_{1}$ obtains a mass given by $\sqrt{2} \mu$. The $\Psi_{2}$ field remains massless and is called a Goldstone Boson [5].

In addition, the Lagrangian density (Eq. 2.8), is expanded in terms of $\Psi(x)$ by Eq. 2.9. This expansion gives mass terms to the fields of the form $c \Psi_{0} A_{\mu} A^{\mu}$, where c is a constant. The fields $\Psi$ and $A_{\mu}$ obtain masses in a way which preserves the renormalizability of the theory. 


\section{Weak Mixing and the CKM Matrix}

The weak interactions conserve color, but do not conserve flavor. When a weak interaction occurs with a $u$-quark, the quark can change flavor to a $d$-quark, $s$-quark or even a $b$-quark. Since no other interactions mix the quark flavors, this is the only possible path for the decay of the second and third generation quarks. This implies that the eigenstates of the weak interactions differ from the mass eigenstates. In order for states to mix, it is necessary that the weak eigenstates be a mixture of the mass eigenstates.

The Cabibbo-Kobayashi-Maskawa matrix (CKM) [14] (Eq. 2.10) describes the mixing of the mass eigenstates to form weak eigenstates. By convention, the weak states of the $+\frac{2}{3}$ charge quarks, denoted $u_{W}, c_{W}$ and $t_{W}$ are defined so that the mass eigenstates are exactly the same as the weak eigenstates. The down, strange and bottom quarks denoted $d_{W}, s_{W}$ and $b_{W}$ are linear combinations of $d, s$ and $b$,

$$
\left(\begin{array}{c}
d_{W} \\
s_{W} \\
b_{W}
\end{array}\right)_{W e a k}=\left(\begin{array}{ccc}
V_{u d} & V_{c d} & V_{t d} \\
V_{u s} & V_{c s} & V_{t s} \\
V_{u b} & V_{c b} & V_{t b}
\end{array}\right)\left(\begin{array}{c}
d \\
s \\
b
\end{array}\right)_{M a s s}
$$

The CKM matrix describes the weak mixing and thus weak decay of the quarks. The matrix elements $V_{i j}$ are in general complex numbers. For a $3 \times 3$ matrix all phases can be transformed away by rotations except one. The remaining phase can accommodate the physics of charge-parity (CP) violation.

The magnitudes of the various matrix elements $\left|V_{i j}\right|$ can be determined by studying the weak decays of the quarks, for example, $\left|V_{u d}\right|$ is determined by studying nuclear $\beta$-decay. 
The least known sector of the CKM matrix is the top quark sector. Measurement of the single top cross section is the only way to obtain a direct measurement of the matrix element $\left|V_{t b}\right|$.

Using the top quark decay channel, $t \rightarrow b l^{+} \nu_{l}$, the matrix element $\left|V_{t b}\right|$ can be constrained [15] as follows,

$$
\frac{\left|V_{t b}\right|^{2}}{\left|V_{t d}\right|^{2}+\left|V_{t s}\right|^{2}+\left|V_{t b}\right|^{2}}=0.99 \pm 0.29 .
$$

The constraint, which is based on a study of $t \bar{t}$ production and decay, has a large uncertainty and depends upon the top quark decay process. If there are more than three families of quarks, or if an exotic top quark decay channel exists, then the above constraint would no longer be valid.

The matrix element $\left|V_{t b}\right|$ can be estimated by imposing the unitarity constraint along with an assumption about the number of generations. If indeed there are only three generations of quarks, one can constrain $\left|V_{t b}\right|$ to be 0.9991 to 0.9994 at the $90 \%$ confidence level [16].

If more than three generations of quarks exist, then the estimates of $\left|V_{t b}\right|$ are considerably poorer with the current constraints. If a fourth generations exists, $0.05 \leq\left|V_{t b}\right| \leq 0.9994$ at the $90 \%$ confidence level [16].

To constrain the matrix element $\left|V_{t b}\right|$ further, quantities which depend upon $\left|V_{t b}\right|$ must be exploited. In Chapter 3, we will see that studying the single top quark production cross sections is an excellent test of the Standard Model predictions and allows for the further study of $\left|V_{t b}\right|$.

\subsection{Standard Model Conclusions}

The Standard Model of particle physics accurately describes or can accommodate all physical processes seen to date with the exception of the solar and atmospheric neutrino data. However, there are a large number of free parameters in the theory 
that must be added in order to explain certain phenomena. This parameterization makes the Standard Model at least an incomplete theory.

Without compelling physics results which indicate a new type of symmetry or interaction, the Standard Model will continue to be the flagship of high energy physics. Nonetheless, to gain an understanding of the free parameters of the Standard Model, such as quark masses, is still a powerful motivation to find a more complete theory. 


\section{CHAPTER 3}

\section{SINGLE TOP THEORY}

\subsection{Top Discovered!}

One of the long awaited results of particle physics was the discovery of the top quark. The top quark was discovered simultaneously by the DØ and CDF experiments at the Fermilab Tevatron in March 1995 [3-4]. Since the discovery, measurement of the top quark mass and the $t \bar{t}$ cross section have been refined with more data and improved methods of calculation. The top quark mass, combining the $\mathrm{D} \varnothing$ and $\mathrm{CDF}$ results is $174.3 \pm 5.1 \mathrm{GeV} / \mathrm{c}^{2}$. The cross section measured by $\mathrm{D} \varnothing$ is $\sigma_{t \bar{t}}=5.5 \pm 1.8 \mathrm{pb}$ and $\sigma_{t \bar{t}}=7.6_{-1.5}^{+1.8} \mathrm{pb}$, by CDF.

The top quark remained elusive for more than two decades after its weak isospin partner, the bottom quark had been discovered [17]. With the top quark discovery, we seek to refine our knowledge of the top quark sector and its interactions. A great deal has been learned already by studying $t \bar{t}$ production [18].

The top quark was discovered via its dominant production mode, which is pair production via the strong interaction. At the Tevatron, the dominant production mode is $q \bar{q} \rightarrow t \bar{t}$. While this mode of production allowed physicists at both CDF and $D \varnothing$ to finally confirm the top quark's existence, little experimental knowledge exists about the top quark's weak interactions. The interaction vertex $W t b$ cannot be easily probed with the strong pair production mode [15]. 
The single top quark can be produced via a $W$-exchange (t-channel) or a $W^{*}$ s-channel process. The asterisk indicates that the $W$ is off its mass shell, that is, it has a mass other than the mass of a real $W$. In the $W^{*}$ s-channel process, the $W$ has enough "mass" to produce a final state of $t \bar{b}$ or $\bar{t} b$.

\section{Reasons to Study Single Top Quark Interactions}

The study of single top quark production can provide insight into the top quark weak interactions, further constrain the top quark mass, and test the validity of the Standard Model. In the following sections, we will explore the reasons for single top quark studies in more detail.

If there are only three generations of quarks, then this has implications regarding the value of the CKM matrix element $\left|V_{t b}\right|$. As described in Section 2.1, if there are only three generations, then unitarity constrains $\left|V_{t b}\right|$ to be very close to unity. If a fourth generation of quarks exists, then the matrix element can have almost any value between zero and unity, determined by the mixing of the elements of the third and fourth generations.

\subsection{Single Top Quark Production Cross Sections}

The cross section for single top production can be determined using

$$
\sigma(p \bar{p} \rightarrow t \bar{b})=6 \Gamma R_{W b}
$$

where $\Gamma$ is the top quark width and $R_{W b}$ is defined as

$R_{W b}=\frac{K}{3} \frac{4 \pi^{2}}{m_{t}} \tau \int_{\tau}^{1} \frac{d x}{x}\left[D_{W / p}\left(x, m_{t}\right) D_{b / p}\left(\tau / x, m_{t}\right)+D_{W / \bar{p}}\left(x, m_{t}\right) D_{b / \bar{p}}\left(\tau / x, m_{t}\right)\right][20]$.

In Eq. 3.2, $\mathrm{K}$ is the QCD enhancement factor, typically less than $2, m_{t}$ is the top quark mass and $D_{W / p / \bar{p}}$ and $D_{b / p / \bar{p}}$ are the proton/anti-proton distribution functions for the $W$ and the $b$-quark, respectively. 


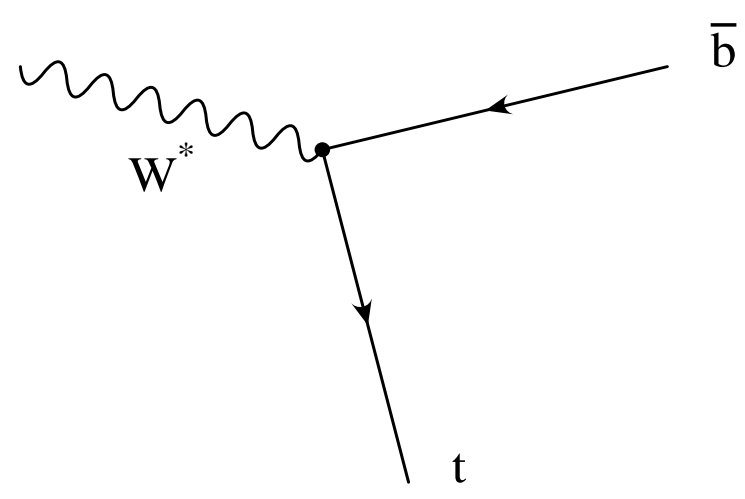

Figure 3.1. The Feynman diagram for inclusive single top production as shown in Eq. 3.1.

The top quark width $(\Gamma)$, when the $b$-quark mass is neglected, can be written in terms of the top quark mass $\left(m_{t}\right)$, the $W$ boson mass $\left(m_{W}\right)$, the CKM matrix element $\left(V_{t b}\right)$ and the weak coupling constant $\mathrm{G}$,

$$
\Gamma=\frac{G m_{t}^{3}}{8 \pi \sqrt{2}}\left|V_{t b}\right|^{2}\left(1-\frac{m_{W}^{2}}{m_{t}^{2}}\right)^{2}\left(1+2 \frac{m_{W}^{2}}{m_{t}^{2}}\right)[21] .
$$

\section{Cross Section for W-Gluon Fusion (t-channel)}

The $W$-gluon fusion or t-channel process allows a study of the charge-current weak interaction of the top quark. The study of single top quark production can provide a direct measurement of the CKM matrix element $\left|V_{t b}\right|$ and is a sensitive indicator of new physics [22-28].

In order to constrain the CKM matrix element $\left|V_{t b}\right|$ and search for new physics, the single top quark cross sections must be well known. The cross section has been calculated at leading order (LO) and found to be $1.84 \mathrm{pb}$ [29-31]. More recently, next-to-leading-order (NLO) calculations have determined a cross section of $1.70 \pm 0.24 \mathrm{pb}[32-33]$.

The single top quark NLO t-channel cross section calculation uses a top quark mass of $175 \mathrm{GeV} / \mathrm{c}^{2}$, the CTEQ4M (NLO) parton distribution functions with the 

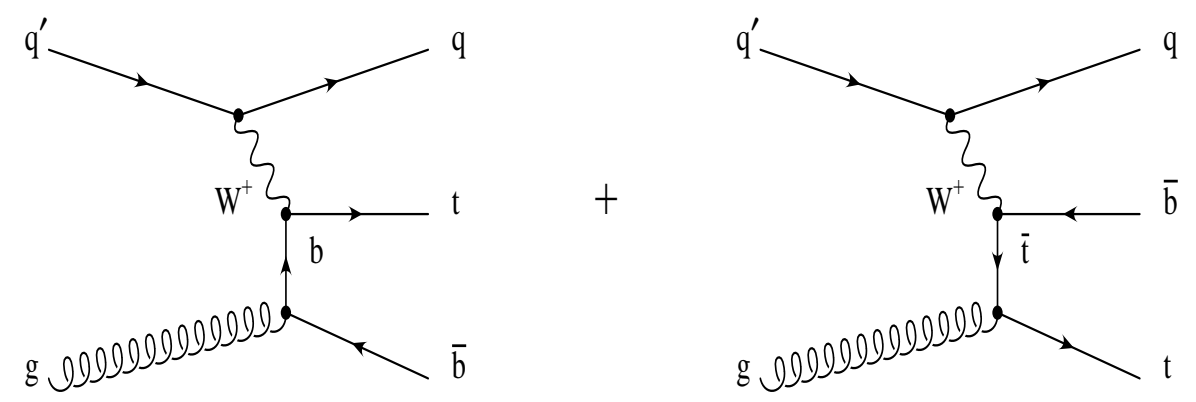

Figure 3.2. The leading order diagrams for single top quark production via W-gluon fusion.

renormalization scale set to $\mathrm{Q}^{2}$ at the light quark vertex and $\mathrm{Q}^{2}+m_{t}$ at the heavy quark vertex. All calculations performed use the $\overline{\mathrm{MS}}$ renormalization scheme.

There are several sources of theoretical uncertainty in the calculation of W-gluon fusion processes: the scale dependence due to neglect of the higher order terms, the top quark mass, and uncertainty in the gluon distribution function. The estimated uncertainty of this calculation is $14 \%$ [33].

\section{Cross Section for $q \bar{q} \rightarrow t \bar{b}$ (s-channel)}

Single top quarks can be produced through s-channel quark-anti-quark annihilation with a highly off-shell $W^{*}$. This production mechanism offers an excellent test of the Standard Model and the best way to measure $\left|V_{t b}\right|$ because of the accuracy of the cross section.

The single top quark s-channel mechanism has been well-studied theoretically. For $\sqrt{s}=1.8 \mathrm{TeV}$, the single top quark s-channel production cross section is predicted to be $0.73 \pm 0.04 \mathrm{pb}[34]$.

In this channel, the calculation uses a top quark mass of $175 \mathrm{GeV} / \mathrm{c}^{2}$, and the factorization and renormalization scales are set to the mass of the virtual $W$-boson. The calculation uses a number of PDFs to estimate the uncertainty. 


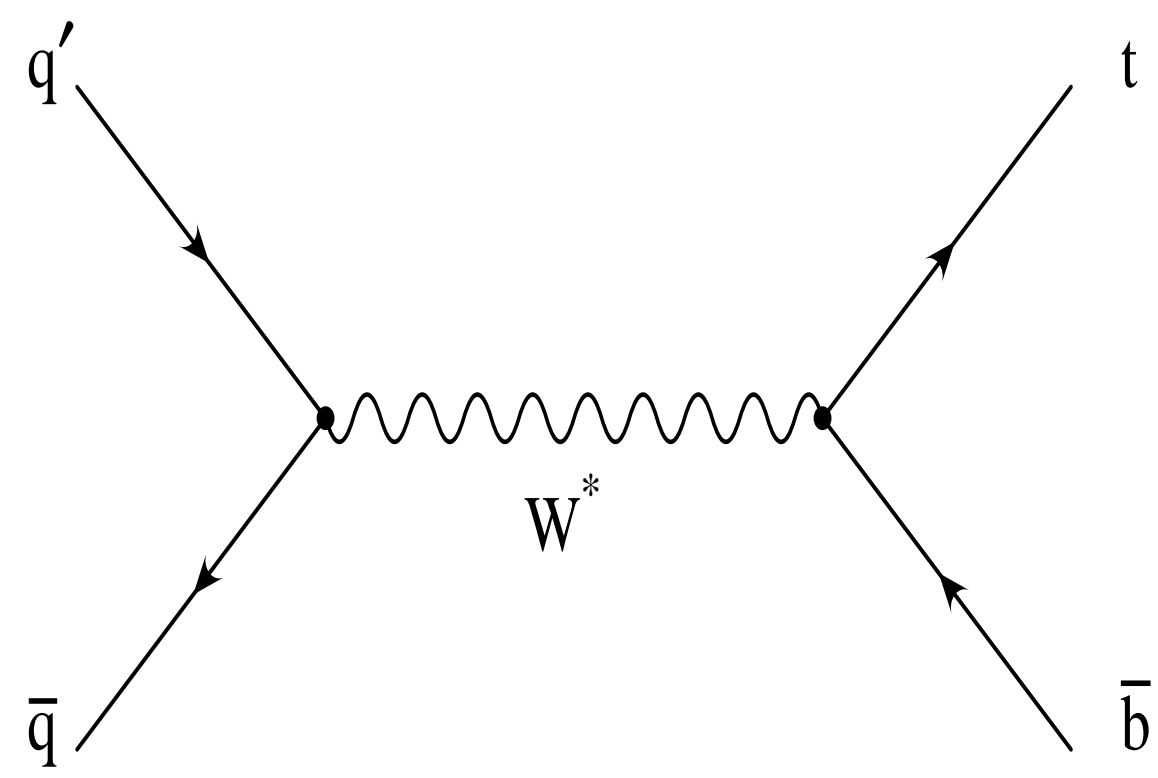

Figure 3.3. The leading order diagrams for single top quark production via the $W^{*}$ (s-channel) process.

The uncertainty in the top quark mass and parton distribution functions contribute significantly to the uncertainty in the cross section. The uncertainty in the parton distributions is estimated to be $\pm 4 \%$. The total uncertainty assigned to the s-channel single top quark cross section is $\pm 6 \%$.

\subsection{Modeling Single Top Quark Production}

It is necessary to correctly model the kinematics of signal events in order to measure a cross section, as the acceptance for a signal depends strongly on the kinematic quantities used to select the events and reduce the backgrounds. The larger the acceptance, the more likely a signal will be observed if it is truly in the data sample. We rely on the Monte Carlo programs CompHEP [36-37] and PYTHIA [38] to model single top production. The CompHEP program is a parton level generator, 
based on exact LO Standard Model matrix elements. CompHEP must be used with a program that models hadronization in order to simulate single top events.

The PYTHIA program does not have a full tree level matrix element calculation for all processes. However, the full matrix element is present for $2 \rightarrow 2$ processes. In the case of $2 \rightarrow 3$ (or more) process, PYTHIA uses a showering algorithm [40].

\section{CompHEP + PYTHIA Model}

CompHEP was used to generate the s-channel and the t-channel single top quark processes. It is possible to add a user defined process into the PYTHIA program [38]. Additional processes from CompHEP have been integrated into PYTHIA in this way.

The single top quark CompHEP simulation uses version PYTHIA 5.7 with JETSET $7.4^{1}$ for these calculations. The generator for the signal events was CompHEP version 3.0. The calculation uses the CTEQ3M parton distribution functions with a scale set equal to the top quark mass for all processes. The events were then modeled with a detector simulation program and then put into a useful format for analysis. The detector and simulation program is discussed in Chapter 4.

In these generated events, the $W$-boson was allowed to decay into $\mu$ or $\tau$ leptons. The $\tau$ lepton has a branching fraction to a $\mu$ lepton of about $17 \%$. There were 100,000 events of this type generated for the s-channel process and 100,000 events generated for the t-channel process.

\section{PYTHIA Monte Carlo Sample}

PYTHIA 6.0.23 has been used to generate single top events [40]. For both processes, we have used the CTEQ3M (NLO) parton distribution functions to model the initial state partons. The top quark mass has been set to $173 \mathrm{GeV} / \mathrm{c}^{2}$.

\footnotetext{
${ }^{1}$ JETSET is used for hadronizing quarks.
} 
The t-channel process is included explicitly in the PYTHIA program. Since we are interested only in events with a muon in the final state, we force the $W$-boson to decay leptonically to either a $\mu$ or a $\tau$ lepton (and its associated neutrino). The minimum $\mathrm{Q}^{2}$ for this process is set to the PYTHIA default, $-\hat{t}^{2}$ [41]. The cross section calculated with PYTHIA is a strong function of the $\mathrm{Q}^{2}$, which indicates that the result is unreliable. However, our results are not based on the PYTHIA cross section. A more significant concern is the observed dependence of some of the kinematic distributions on the value of $\mathrm{Q}^{2}$. We find that the $\eta$ distributions of the spectator $b$-quark in this process differ between CompHEP and PYTHIA as shown in Fig. 3.3. When $p_{T}$ cuts are made on the $p_{T}$ of the spectator quark and the final state quark, the agreement is improved as shown in Fig. 3.3. In Fig. 3.3, the spectator $b$-quark was required to have $p_{T}>15 \mathrm{GeV} / \mathrm{c}$ and the final state light quark was required to have $p_{T}>10 \mathrm{GeV} / \mathrm{c}$. The cause of the differences has led to some debate [42]. We note, however, that both CompHEP and PYTHIA provide approximate calculation of observables.

For the t-channel process, we use PYTHIA subprocess number 83,

$$
f+q \rightarrow f^{\prime}+Q \text { (massive) }
$$

The $\mathrm{W}$ was allowed to decay to either a $\mu$ or a $\tau$ lepton and its associated neutrino. A typical event summary for this subprocess is shown in Table 3.1. PYTHIA generates the process $p \bar{p} \rightarrow t q b$, where there is always a b-quark in the final state.

Some kinematic quantities from parton level studies are shown in Fig. 3.3. The quantities are shown for the CompHEP and PYTHIA models. One can see that both model the $W$ from the top quark and the final state quark very well, but are vastly different in the $p_{T}$ and $\eta$ distributions of the final state $b$-quarks. It is certainly true that these quarks will be the least well modeled because they are lower energy 
Table 3.1. A typical event from PYTHIA for single top quark t-channel production at the Fermilab Tevatron. The first column is the particle index number (I). The second and third columns are the particle name (particle) and the index of the "mother" particle (orig). The remaining columns are the particle's momenta (p_x,p_y,p_z) in $\mathrm{GeV} / \mathrm{c}$, energy (E) in $\mathrm{GeV}$ and mass $(\mathrm{m})$ in $\mathrm{GeV} / \mathrm{c}^{2}$.

\begin{tabular}{|c|c|c|c|c|c|c|c|}
\hline I & particle & orig & $p_{-} x$ & p_y & p_z & $\mathrm{E}$ & $\mathrm{m}$ \\
\hline 1 & $! p+!$ & 0 & 0.000 & 0.000 & 900.000 & 900.000 & 0.938 \\
\hline 2 & !pbar-! & 0 & 0.000 & 0.000 & -900.000 & 900.000 & 0.938 \\
\hline \multicolumn{8}{|c|}{ 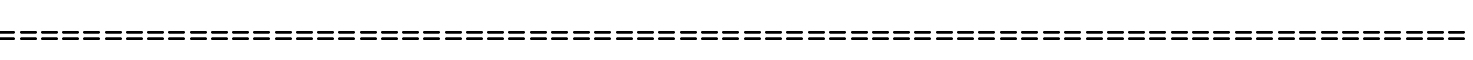 } \\
\hline 3 & $\lg !$ & 1 & 0.043 & -0.783 & 205.074 & 205.075 & 0.000 \\
\hline 4 & !dbar! & 2 & -0.592 & 0.115 & -253.113 & 253.113 & 0.000 \\
\hline 5 & !b! & 3 & -3.663 & 7.226 & 84.319 & 84.708 & 0.000 \\
\hline 6 & !dbar! & 4 & 0.528 & -0.968 & -248.635 & 248.637 & 0.000 \\
\hline 7 & !t! & 0 & 57.492 & 9.256 & -27.798 & 183.941 & 172.251 \\
\hline 8 & !ubar! & 0 & -60.626 & -2.998 & -136.517 & 149.404 & 0.006 \\
\hline 9 & $! W+!$ & 7 & 58.985 & -56.540 & -13.129 & 114.079 & 78.522 \\
\hline 10 & $! b !$ & 7 & -7.682 & 65.173 & -20.840 & 69.035 & 5.000 \\
\hline 11 & !mu+! & 9 & 36.297 & -2.930 & 25.294 & 44.338 & 0.106 \\
\hline 12 & !nu_mu! & 9 & 22.643 & -53.545 & -38.422 & 69.685 & 0.000 \\
\hline
\end{tabular}




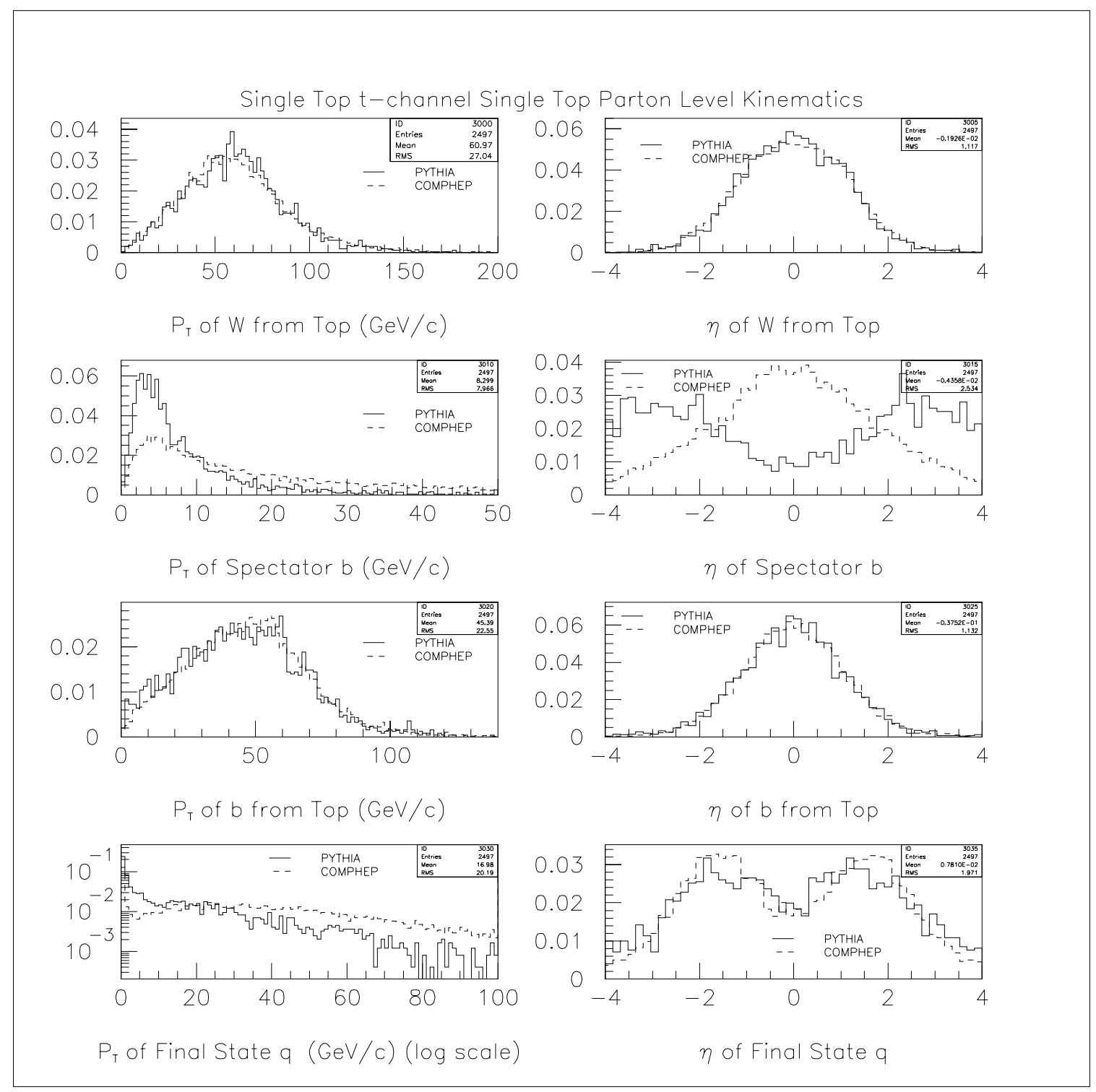

Figure 3.4. Some kinematic quantities for the single top quark t-channel processes with CompHEP and PYTHIA. 


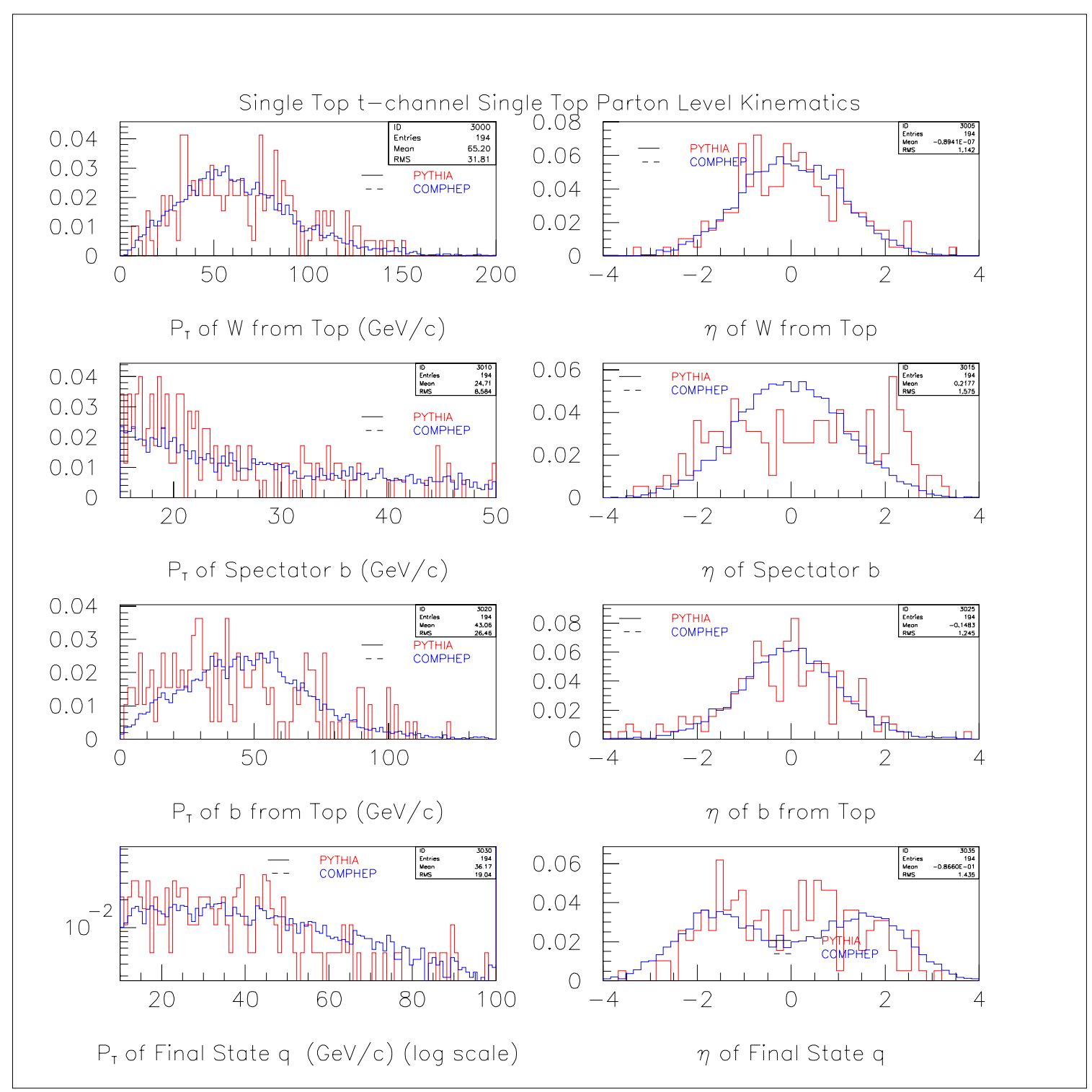

Figure 3.5. Some kinematic quantities for the single top quark t-channel processes with CompHEP and PYTHIA. Here, cuts have been applied to remove the low- $p_{T}$ spectator quarks (below $15 \mathrm{GeV} / \mathrm{c}$ ) and low- $p_{T}$ final state light quarks (below 10 $\mathrm{GeV} / \mathrm{c}$ ). The agreement is much better between CompHEP and PYTHIA. 
constituents which are modeled differently. It is important that the models accurately predict the most energetic partons in the event and this is certainly true here.

PYTHIA does not have an explicit implementation of the s-channel single top process $\left(W^{*}\right)$. However, the process can be generated as follows: generate $p \bar{p} \rightarrow W$ events with $\sqrt{\hat{s}}>\sqrt{m_{t}^{2}+m_{b}^{2}}$, that is, a reaction mediated by a highly off-shell $W$-boson. We then allow the off-shell $W$ to decay to all final states, but keep only the events in which the $W^{*}$ decays into a top and a bottom quark.

We use PYTHIA subprocess $2, f+\bar{f} \rightarrow W^{ \pm}$with the center of mass energy slightly less than that required to produce a top and bottom quark, thus allowing for "threshold" effects due to the widths of the top and bottom quark. To produce a $\mu+$ jets sample, we retain only events where the $W$ from the top quark decays into either $\mu$ or $\tau$ leptons. The $W$ is allowed to decay to a $\tau$ lepton because the $\tau$ lepton has a significant branching fraction to $\mu$ leptons. Parton level kinematic distributions for CompHEP and PYTHIA are shown in Fig. 3.3. A typical s-channel event summary is shown in Table 3.2.

\section{Single Top Signal and Background Predicted Yields}

In order to further motivate the single top searches, it is useful to consider the theoretical predictions for the signal and background processes. The backgrounds that we consider are $t \bar{t}$ and $W+$ jets. There are additional background processes, but the $W+$ jets process is the dominant background to the $\mu+$ jets channel. We use the single top cross sections given above and the NLO $t \bar{t}$ cross section of $5.52 \mathrm{pb}$ [35].

For the $W+$ jets processes, we use CompHEP [36-37] to calculate a leading order cross section for three different final states according to the flavor of the jets in the process. For light quarks ( $u$ - or $d$-quark) $(W+\mathrm{ud})$ and gluon jets the CompHEP cross section is $966 \mathrm{pb}$. For jets with $c$ or $s$ quark jets $(W+\mathrm{cs})$, the CompHEP cross 
Table 3.2. A typical event from PYTHIA for single top quark s-channel production at the Fermilab Tevatron. The first column is the particle index number (I). The second and third columns are the particle name (particle) and the index of the "mother" particle (orig). The remaining columns are the particle's momenta (p_x,p_y,p_z) in $\mathrm{GeV} / \mathrm{c}$, energy (E) in $\mathrm{GeV}$ and mass $(\mathrm{m})$ in $\mathrm{GeV} / \mathrm{c}^{2}$.

\begin{tabular}{|c|c|c|c|c|c|c|c|}
\hline I & particle & orig & p_x & p_y & p_z & $E$ & $\mathrm{~m}$ \\
\hline 1 & $! p+!$ & 0 & 0.000 & 0.000 & 900.000 & 900.000 & 0.938 \\
\hline 2 & !pbar-! & 0 & 0.000 & 0.000 & -900.000 & 900.000 & 0.938 \\
\hline 3 & ! u! & 1 & 0.188 & 0.280 & 327.646 & 327.646 & 0.000 \\
\hline 4 & !dbar! & 2 & 0.218 & 0.198 & -120.601 & 120.601 & 0.000 \\
\hline 5 & $! \mathrm{u} !$ & 3 & 0.204 & -1.383 & 169.982 & 169.988 & 0.000 \\
\hline 6 & !dbar! & 4 & -92.284 & 11.876 & -31.434 & 98.211 & 0.000 \\
\hline 7 & $! W+!$ & 0 & -92.079 & 10.493 & 138.548 & 268.198 & 210.110 \\
\hline 8 & !bbar! & 7 & -0.760 & -9.790 & -15.063 & 18.663 & 5.000 \\
\hline 9 & !t! & 7 & -91.319 & 20.283 & 153.611 & 249.535 & 172.976 \\
\hline 10 & $! W+!$ & 9 & -45.244 & -50.212 & 43.116 & 110.603 & 76.196 \\
\hline 11 & $! b !$ & 9 & -45.950 & 70.407 & 110.187 & 138.690 & 5.000 \\
\hline 12 & $! m u+!$ & 10 & 7.802 & -39.276 & 39.496 & 56.244 & 0.106 \\
\hline 13 & !nu_mu! & 10 & -52.718 & -9.731 & 3.768 & 53.741 & 0.000 \\
\hline
\end{tabular}




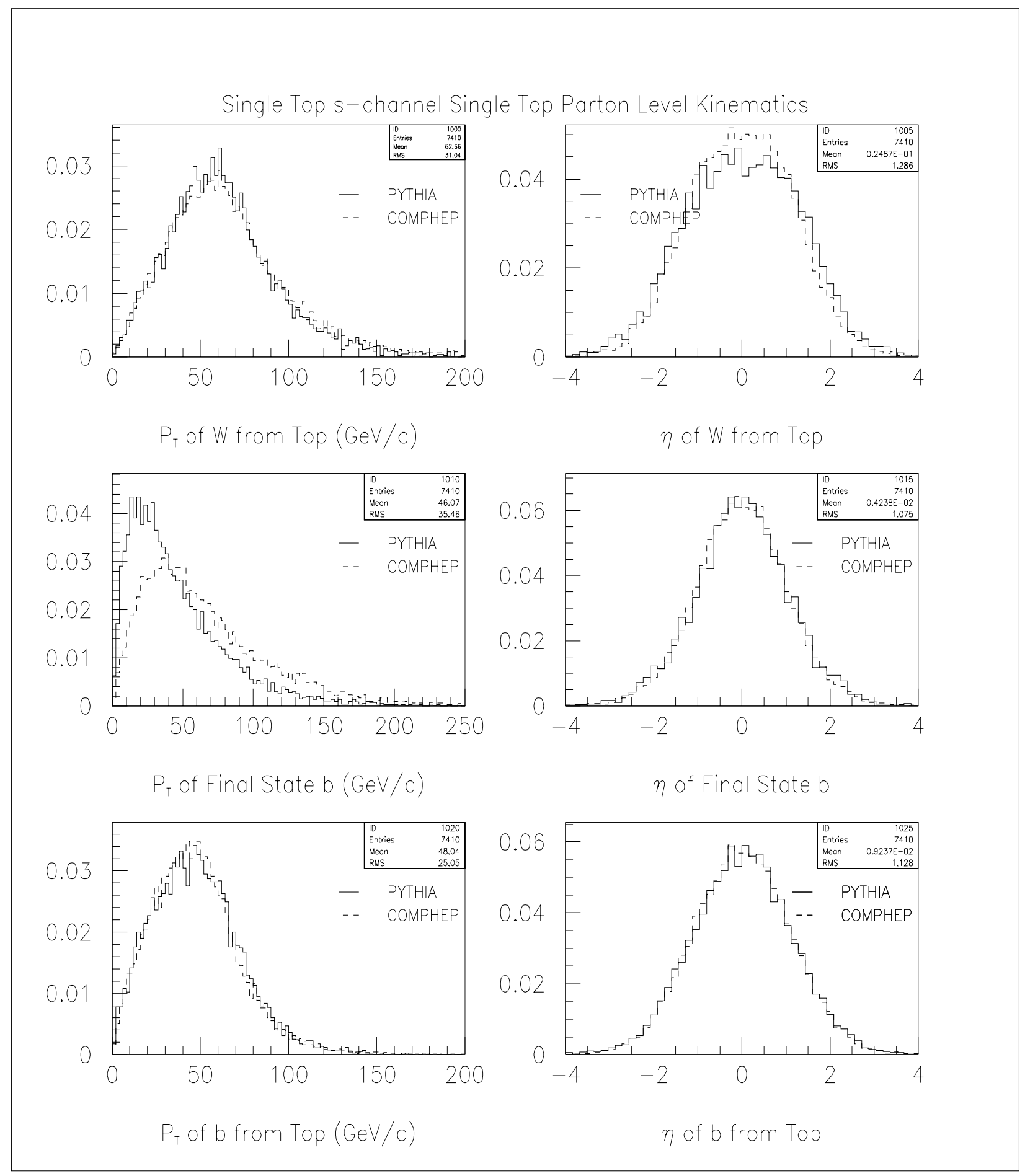

Figure 3.6. Some kinematic quantities for the single top quark s-channel processes with CompHEP and PYTHIA. 
Table 3.3. Predicted event yields for single top and the primary backgrounds. The yield is for an integrated luminosity of $100 \mathrm{pb}^{-1}$.

\begin{tabular}{|l|c|c|c|c|}
\hline \multicolumn{2}{|c|}{ Process } & Total & $\mu+$ jet & $\mu+$ jets $/ \mu$ \\
\hline \multirow{4}{*}{ Signal } & $\mathrm{tb}$ & 72 & 9 & 2 \\
\cline { 2 - 5 } & $\mathrm{qtb}$ & 173 & 23 & 4 \\
\cline { 2 - 5 } & $\bar{t}$ & 522 & 80 & 16 \\
\cline { 2 - 6 } & $W+\mathrm{ud}$ & 96,600 & 12,558 & 60 \\
\cline { 2 - 6 } & $W+\mathrm{cs}$ & 2790 & 362 & 18 \\
\cline { 2 - 6 } & $W+\mathrm{bb}$ & 726 & 94 & 18 \\
\hline
\end{tabular}

section is $27.9 \mathrm{pb}$. For $b$-quark jets $(W+\mathrm{bb})$, the CompHEP cross section is 7.26. The uncertainty on these cross sections is about $30 \%$.

For an integrated luminosity of $100 \mathrm{pb}^{-1}$, the predicted yields for the single top process and the above backgrounds is given in Table 3.3.

One must make some assumptions about the light quark jets and their contribution to the $\mu+$ jets $/ \mu$ channel. We have assumed that the $W+$ light background has a branching fraction of $0.5 \%$ to the $\mu$ tagged channel. For the $W+$ cs background, we have assumed a branching fraction of $5 \%$ to the $\mu$ tagged channel. These are estimates based on a Run II study [39]. For all other channels, the branching fraction is approximately $10 \%$ per $b$-quark jet.

Although the number of expected signal events is small, the possibility of single top observation warrants further study. We note that the $t \bar{t}$ channel was observable with slightly more than twice the number of events than predicted for single top.

Missing from Table 3.3 is the background from QCD multi-jet processes; these backgrounds are not negligible. Unfortunately, estimates of event yields for these 
processes are difficult to calculate. However, the QCD multi-jet background is calculated for the specific selection criteria of this analysis in Section 6.5.1.

\subsection{Single Top Quark Measurements}

As stated above in Section 3.1, the study of single top quark production offers many interesting opportunities for physics measurements. A cross section can be used to obtain limits for $\left|V_{t b}\right|$.

Since new physics may be found at an energy scale near the top quark mass, single top may also shed light on new physics.

\section{Measurement of $\left|V_{t b}\right|$}

If the experimental measurement of the single top cross section deviates from the Standard Model predictions it can indicate a deviation in $\left|V_{t b}\right|$ or a deviation in the top quark partial width $\Gamma$. As an aside, the partial width is determined by the summing the widths of the individual decay channels. If the top quark decays to quarks lighter than a $b$-quark, the contributions from these other channels contribute to the width. For a top mass of $175 \mathrm{GeV} / \mathrm{c}^{2}$, the top quark width is predicted to be $1.5 \mathrm{GeV}[44]$.

The interpretation of the result depends on the ratio $\frac{\sigma_{m}}{\sigma_{t h}}$. Here, $\sigma_{m}$ is the measured cross section for single top and $\sigma_{t h}$ is the theoretical cross section for single top. If the ratio is smaller than one, indicating a cross section lower than Standard Model expectations, the interpretation will be a deviation of the CKM matrix element $\left|V_{t b}\right|$ from unity. The CKM matrix elements can be no larger than unity. The measured cross section is related to $\left|V_{t b}\right|$ and $\Gamma$ by

$$
\sigma_{m}=\left|V_{t b}\right|^{2} \sigma_{t h}=c \Gamma \sigma_{t h}
$$

where $c$ is a constant. 
If an enhancement is seen, the ratio is larger than one and the likely explanation for the deviation will be new physics, see Section 3.4. If the ratio is exactly one, then the Standard Model will have passed yet another important test.

\section{New Physics}

There have been numerous publications which speculate about the single top quark production cross section. Discussions of anomalous gluon-top quark couplings are discussed in a number of references [45-51].

One quantity of interest in the study of anomalous couplings is the form of the $W t b$ coupling. It is possible to hypothesize a $(V+A)$ interaction in the $W t b$ coupling. New physics can be probed by considering more general operators for the $W t b$ coupling,

$$
\frac{g}{\sqrt{2}}\left(W_{\mu}^{-} \bar{b} \gamma_{\mu}\left(f_{L} \frac{1}{2}\left(1-\gamma_{5}\right)+f_{R} \frac{1}{2}\left(1+\gamma_{5}\right)\right) t\right)
$$

In Eq. 3.5, the $W, t$ and $b$ particles are represented by the fields $W_{\mu}^{-}, t$ and $\bar{b}$, respectively. $\gamma_{5}$ and $\gamma_{\mu}$ are Dirac matrices. The form factors $f_{L}$ and $f_{R}$ parameterize dependence on the $(V-A)$ and $(V+A)$ terms respectively. In the Standard Model, the form factor $f_{L}=1$ and $f_{R}=0$ [59].

The single top production mechanism is the only method which can be used to probe the $W t b$ coupling. These measurements require a precision measurement of the cross section and are not expected to be measured until Run II. Run II is the next collider run of the Fermilab Tevatron, which is expected to deliver 20 times the luminosity used for this analysis.

Single top quark physics can also point to new contact interaction terms and new strong dynamics. There are a number of references which discuss the many different possible manifestations of new physics in single top quark production [52-58].

Single top quark physics also has implications regarding supersymmetric models and has been studied in Ref. [60-62]. 


\section{CHAPTER 4}

\section{THE DØ DETECTOR}

\subsection{Introduction}

At the Fermilab Tevatron, the collision of proton-anti-proton beams at a center of mass energy of $1.8 \mathrm{TeV}$ opens windows to exciting new areas of high energy physics. This center of mass energy is currently the highest available in the world. It is these new areas of physics the $\mathrm{D} \varnothing$ detector was designed to observe. In particular, the D $\varnothing$ detector was designed to study high mass states and large $p_{T}$ phenomena.

In order to be sensitive to phenomena with high mass states or low cross sections, physicists must carefully study and optimize the detector so that they obtain the best signal to background ratio possible. The signal processes from $W$ and $Z$ bosons and the top quark are predicted to have an appreciable leptonic branching fraction, which makes their observation more likely because the dominant background has a relatively small leptonic branching fraction. In the all-hadronic final state the QCD background overwhelms the signal process with the same final states.

The DØ detector design goals were: excellent detection of electrons and muons, good energy resolution and a precision measurement of energy lost due to neutrinos, called missing transverse energy $\left(\mathscr{E}_{T}\right)$.

The DØ detector features a stable, unit gain, hermetic, finely segmented and radiation-hard calorimeter for the precise measurement of transverse energy, $E_{T}$. For detection of muons, the detector has three layers of tracking chamber and a 
thick magnetic iron absorber to measure the muon momentum from the deflection of its track and to minimize background contamination from hadronic punchthrough. Near the interaction region, the detector contains a non-magnetic tracking system.

The $D \varnothing$ detector sits upon a platform for insertion into the collision hall. The Movable Counting House $(\mathrm{MCH})$ serves as the detector read out center for the detector. From the MCH, data are served to processor nodes in the detector control room for observation and storage. However, before the DØ detector can record any interesting high energy events, protons and anti-protons must be accelerated to 900 $\mathrm{GeV}$ and made to collide in the DØ collision hall.

\subsection{The Tevatron}

The $\mathrm{D} \varnothing$ detector is used to record events that have been produced by the Fermilab Tevatron. The Fermilab Tevatron is a proton-anti-proton synchrotron capable of delivering beams of protons and anti-protons which collide with a center of mass energy of $\sqrt{s}=1.8 \mathrm{TeV}$. A large chain of events occur before collisions are observed at $\mathrm{D} \varnothing$.

The first step in delivering beam to the collision halls is the acceleration of protons to generate anti-protons. The process begins with a Cockroft-Walton accelerator where negatively charged hydrogen ions are accelerated by a two stage linear accelerator to an energy of $400 \mathrm{MeV}$. The electrons are removed from the hydrogen when the ion passes through a carbon foil and is simultaneously injected into the Booster, the next stage of acceleration. The beam is pulsed as it enters the Booster.

Any $\mathrm{H}^{-}$or $\mathrm{H}^{0}$ atoms are removed from the beam with a magnetic field which selects them out and dumps them from the beam. This eliminates anything other than protons from the beam. 


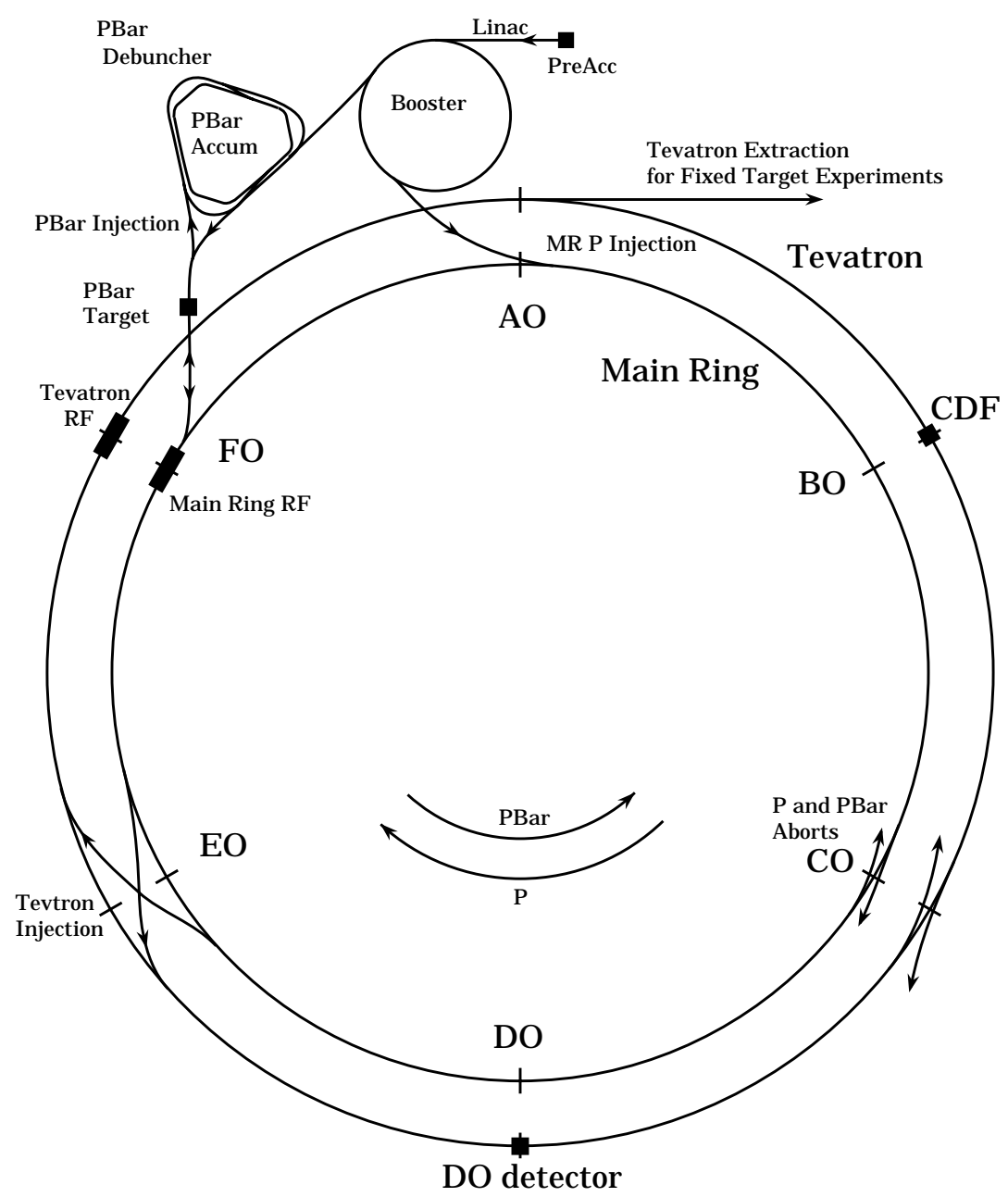

Figure 4.1. The Fermilab Tevatron proton/anti-proton synchrotron.

The Booster is a fast cycling proton synchrotron which takes the $400 \mathrm{MeV}$ protons from the linear accelerator and accelerates them to $8 \mathrm{GeV}$ in preparation for Main Ring injection. The entire Booster stage process takes only $33 \mathrm{msec}$.

With a radius of 1000 meters, the Main Ring serves two purposes: Generation of anti-protons and injection of protons and anti-protons into Tevatron. The Main Ring is a $400 \mathrm{GeV}$ synchrotron. For generating anti-protons, the Main Ring is a source of $120 \mathrm{GeV}$ protons. For Tevatron injection, the Main Ring delivers $150 \mathrm{GeV}$ protons. 
Anti-protons are generated by colliding $120 \mathrm{GeV}$ protons with a fixed target, usually nickel. For every proton that collides with the nickel plate, only 1 in 100,000 will produce an anti-proton. The anti-protons are captured and cooled stochastically in the anti-proton accumulator. The cooling occurs by an accelerator feedback loop which determines the transverse momentum of the beam and then makes corrections for transverse momentum at later times. Once enough anti-protons have been produced and cooled, they can be injected into the Main Ring for later injection into the Tevatron and finally, collisions.

The protons and anti-protons are accelerated to $150 \mathrm{GeV}$ in the Main Ring and injected into the Tevatron. Because protons and anti-protons are oppositely charged and have the same mass, the same magnets and acceleration devices can be used to steer and accelerate the beams. Consequently, protons move in the opposite direction with respect to the anti-protons.

For Tevatron collisions, a continuous beam is not practical, so a number of bunches, typically six, were injected into the Tevatron. The number of bunches is actually determined very early in the acceleration stage ${ }^{1}$. Each bunch of protons contained approximately $200 \times 10^{9}$ protons; each anti-proton bunch contained approximately $50 \times 10^{9}$ anti-protons. Typically, the longitudinal extent of the bunch was $30 \mathrm{~cm}$ and transversely, the beam was localized to about $30 \mu \mathrm{m}$. When all six proton and six anti-proton bunches had been injected, the Tevatron accelerated each bunch to an energy of $900 \mathrm{GeV}$. The resulting beam crossing time is $3.5 \mu \mathrm{sec}$. The peak luminosity at DØ during Run $1 \mathrm{~b}$ exceeded $20 \times 10^{30} \mathrm{~cm}^{-2} \mathrm{sec}^{-1}$. The Tevatron was designed for a peak luminosity of $210 \times 10^{30} \mathrm{~cm}^{-2} \mathrm{sec}^{-1}$ [16].

\footnotetext{
${ }^{1}$ The number of bunches is determined during the Booster stage.
} 


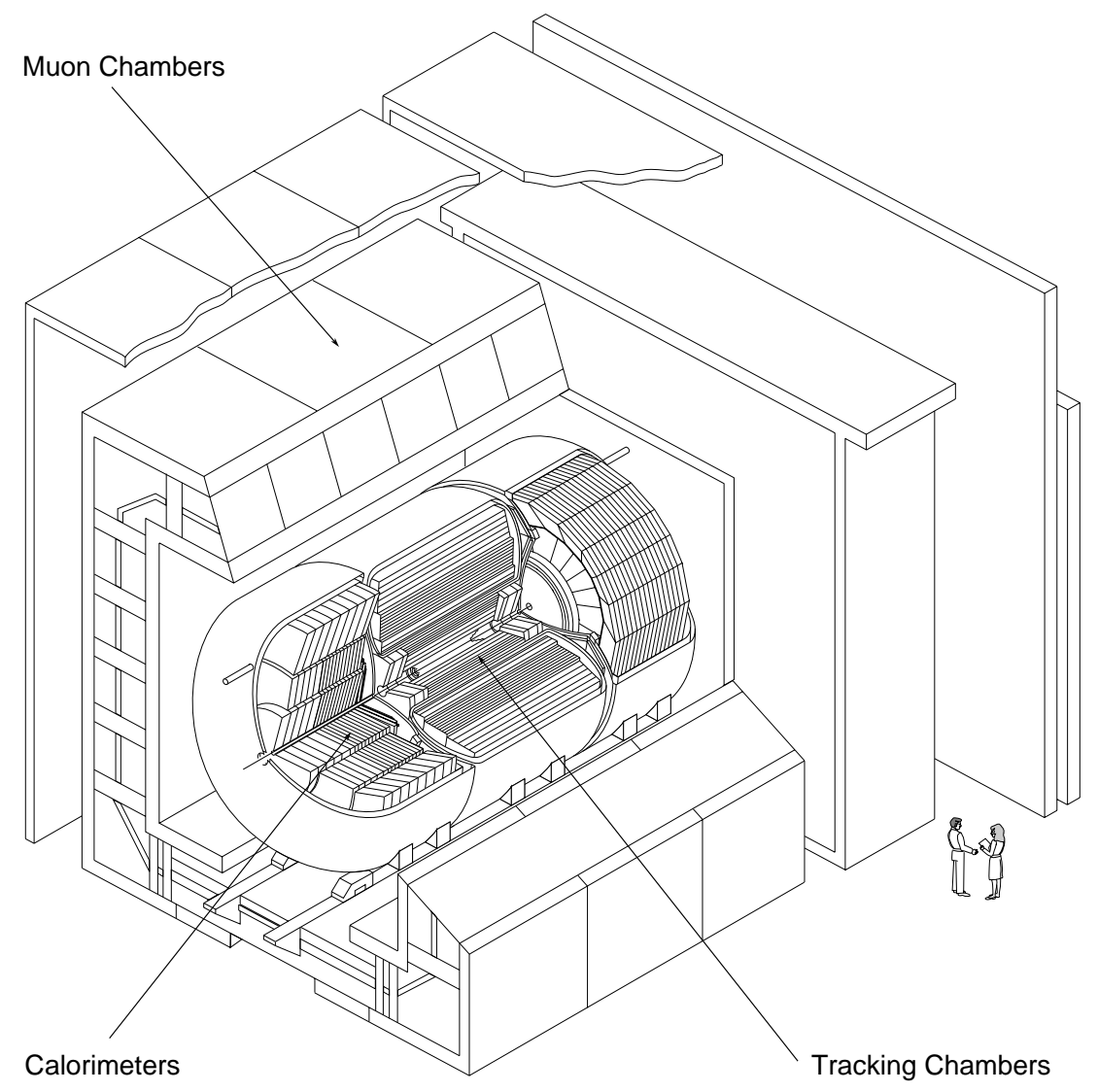

Figure 4.2. The D $\varnothing$ detector.

\subsection{Coordinate System}

The DØ Collaboration has defined a standard coordinate system for use in analyses. The system defines $+\hat{x}$ be a unit vector which points radially outward from the center of the accelerator ring, $+\hat{y}$ to be a unit vector pointing upward from the beam pipe and $+\hat{z}$ be defined so as to make the coordinate system right-handed.

With this requirement, the $+\hat{z}$ direction is to the South; the direction from which protons come. The anti-protons move in the $-\hat{z}$ direction. Frequently, spherical coordinates are used. The polar angle $\theta$ is defined as the angle from the $+\hat{z}$ axis and the azimuthal angle $\phi$ defined as the angle about the $+\hat{z}$ axis in the $\mathrm{x}-\mathrm{y}$ plane. At 
$\phi=0$ the direction is along the $+\hat{x}$ axis and at $\phi=\frac{\pi}{2}$ the direction is along the $+\hat{y}$ axis.

A more useful angular quantity than $\theta$ from a physical standpoint is the rapidity, defined by $y=\frac{1}{2} \ln \left(\left(E+p_{z}\right) /\left(E-p_{z}\right)\right)$, which can be approximated by the pseudorapidity in the limit that a mass of a particle is negligible, i.e. $p \ll m$. The pseudorapidity is thus defined as $\eta=-\ln (\tan (\theta / 2))$. With this definition of $\eta$, the angle $\theta=\sin ^{-1}(1 / \cosh (\eta))$. A pseudorapidity of 0 is in the $-\hat{y}$ direction. A pseudorapidity of $\infty$ is in the $+\hat{z}$ direction.

\subsection{Central Detectors}

The central DØ detector serves to track charged particles and determine the $\mathrm{z}$ coordinate of the collision point (the z-vertex). There are four subsystems which comprise the Central Detector: Vertex Drift Chamber (VTX), Transition Radiation Detector (TRD), Central Drift Chamber (CDC) and Forward Drift Chambers (FDC). The Run I D $\varnothing$ detector did not have a central magnetic field. The primary considerations for optimization of the Central Detectors were good two-track resolution, high efficiency and good ionization energy measurement.

Located just outside the beryllium beam pipe, the vertex chamber (VTX) is composed of one layer of 16 cells and two outer layers of 32 cells with eight sense wires per cell. The VTX provides measurement of the $\mathrm{r}-\phi$ coordinate. Adjacent wires are staggered to resolved left-right ambiguities. Read out of the VTX provides a measurement of the $z$-vertex.

Between the VTX and the CDC, the TRD assists in the identification of electrons. As relativistic particles pass through materials with different dielectric constants, the particles emit X-rays. The detected X-rays aid in electron identification. 


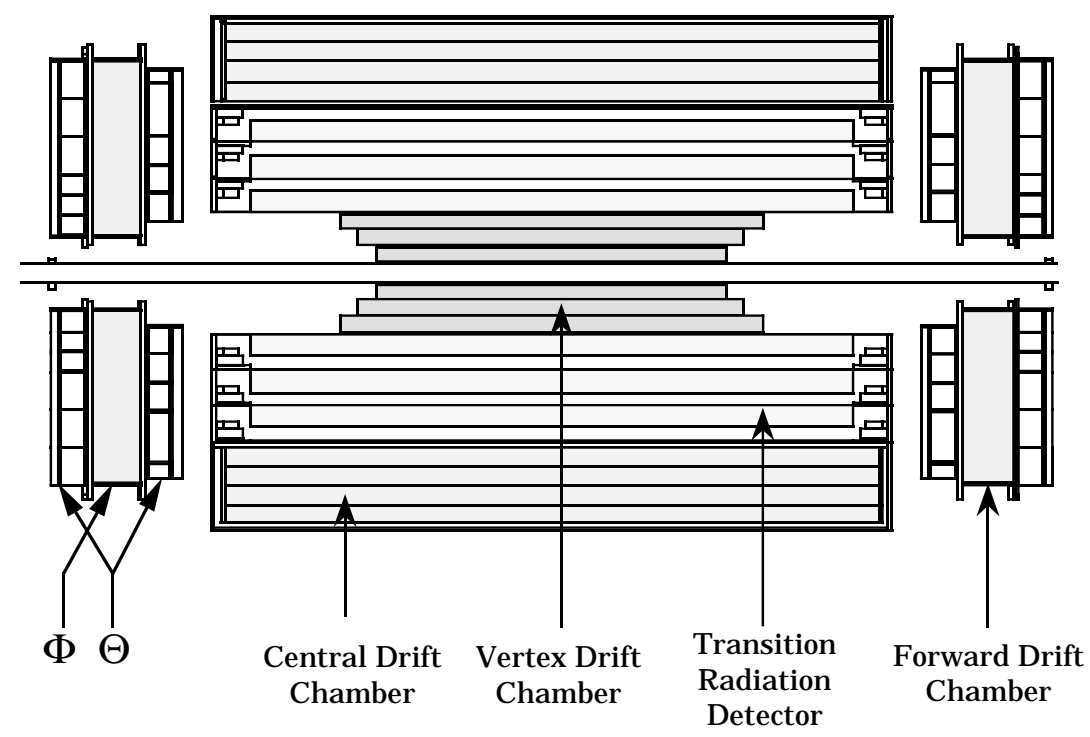

Figure 4.3. The central detectors at D $\varnothing$.

The Central Drift Chamber lies just before Central Calorimetry. The Central Drift Chamber is a cylinder with four concentric rings of 32 azimuthal cells per ring. The field inside the drift chamber is $620 \mathrm{~V} / \mathrm{cm}$ and the drift velocity is $34 \frac{\mu \mathrm{m}}{\mathrm{ns}}$. The gas inside the drift chamber is composed of argon, methane, carbon dioxide and a trace of water with a gain of $2 \times 10^{4}$ for the inner cells and $6 \times 10^{4}$ in the outer cells.

The Forward Drift Chambers are placed at either end of the Central Drift Chamber and extend coverage out to $\theta \approx 5^{\circ}$. The FDC is composed of three separate modules. Two FDC modules measure the angle $\theta$ and one module measures the angle $\phi$, see Fig. 4.4.

\subsection{Calorimetry}

Central to the physics program at $D \varnothing$ is the highly segmented, hermetic liquid argon calorimeter. The central calorimeter was designed to provide good energy resolution for electrons, photons and jets. Neutrinos cannot be detected with an acceptable efficiency; however, the presence of neutrinos can be inferred from the imbalance in the transverse energy of an event using the calorimeter. 


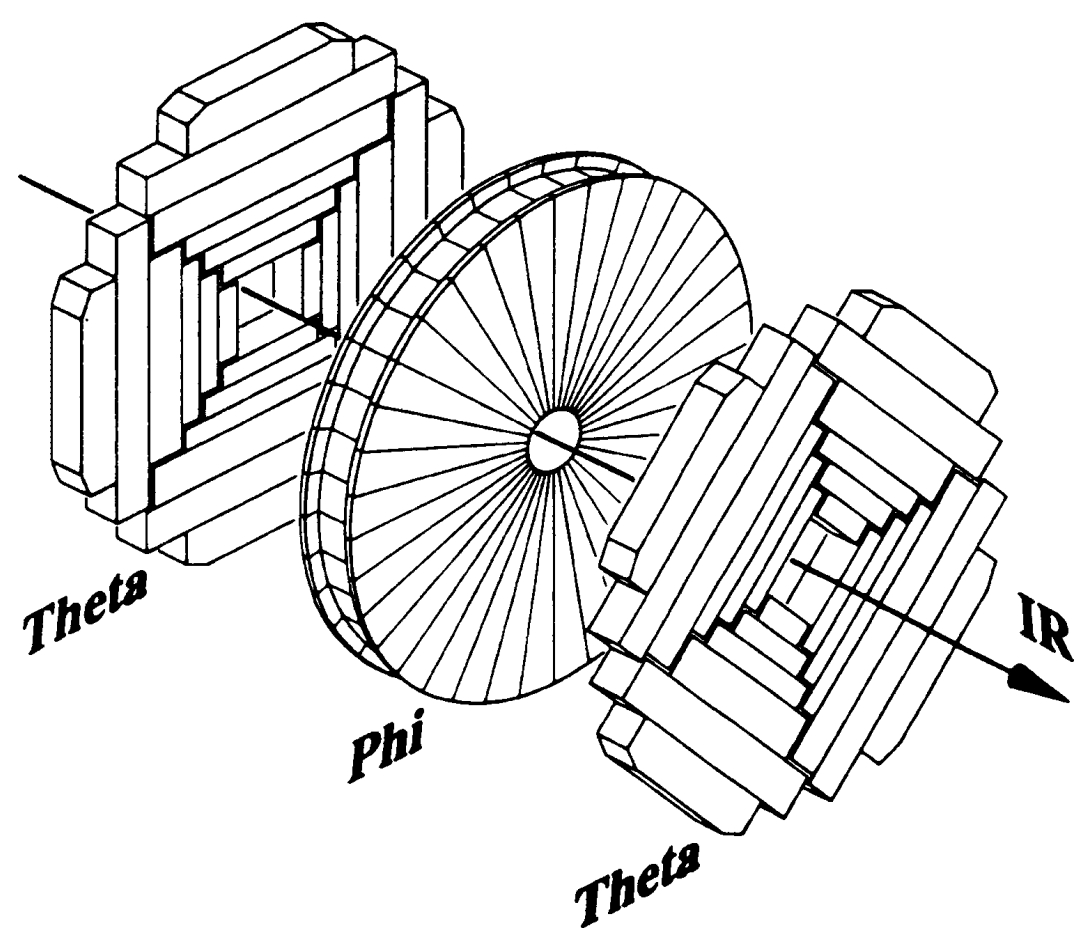

Figure 4.4. The forward drift chambers for the $D \emptyset$ detector. The forward drift chambers consists of a two modules for theta measurement between a module for phi measurement.

The active medium, liquid argon, was chosen for its unit gain, stable calibration, the ease of making diverse calorimeter cells, its radiation-hardness and the relatively low cost of read out electronics. However, liquid argon requires complex cryogenic systems and cryostats, making instrumentation in these areas impossible and access to the calorimeter cells extremely difficult.

The calorimeter works by sampling particle energies as the particles pass between absorber material. The passage of particles through the argon ionizes the argon and initiates a cascade of charged particles which gives rise to a current on a high voltage pad.

In order to provide access to critical portions of the inner detector, the calorimeter is divided into three sections. The central calorimeter (CC) covers roughly $|\eta| \leq 1.2$. 
Two end calorimeters (EC) complete the $|\eta| \leq 4$. coverage. The boundary between the EC and CC that was most optimal for determination of missing transverse energy $\left(\mathscr{E}_{T}\right)$ was shown to be a configuration which is perpendicular to the beamline.

Both central and end calorimeters have three regions of segmentation: electromagnetic, fine-hadronic and coarse-hadronic. The electromagnetic calorimeter is the most highly segmented. The absorber in the electromagnetic segment is composed of thin uranium plates. The fine-hadronic has thicker uranium plates and the course hadronic absorber is composed of copper or stainless steel plates.

\section{Central Calorimeter}

The Central Calorimeter (CC) lies in the central region of the D $\varnothing$ detector, covering the $|\eta|<1.2$ range of the detector. The Central Calorimeter is composed of three concentric rings of detectors: CCEM (Electromagnetic), CCFH (Fine Hadronic) and CCCH (Coarse Hadronic). From the center of the cryostats out, there are $32 \mathrm{CCEM}$ modules in $\phi, 16 \mathrm{CCFH}$ modules and $16 \mathrm{CCCH}$ modules in the outermost ring. The modules are staggered in $\phi$ so that no two module boundaries in adjacent layers are aligned. Each module is finely segmented, typically $0.1 \times 0.1$ in $\eta \times \phi$ space, and longitudinally. The segmentation is finer in the CCEM modules for greater electromagnetic shower resolution. The resolutions vary by module. Each cell in the modules is independently read out.

\section{Central Calorimeter Electromagnetic Modules (CCEM)}

The 32 uranium absorber CCEM modules have 21 radiation lengths $^{2}$ and cover the full interaction region along the $\mathrm{z}$-axis and about 0.2 radians in azimuth. The modules are read out in four radiation length depths: Two, two, seven, and ten. The

\footnotetext{
${ }^{2} \mathrm{~A}$ radiation length is the distance an electron travels such that, due to energy loss to bremsstrahlung, its energy falls by a factor of $e^{-1}$ from its initial energy.
} 


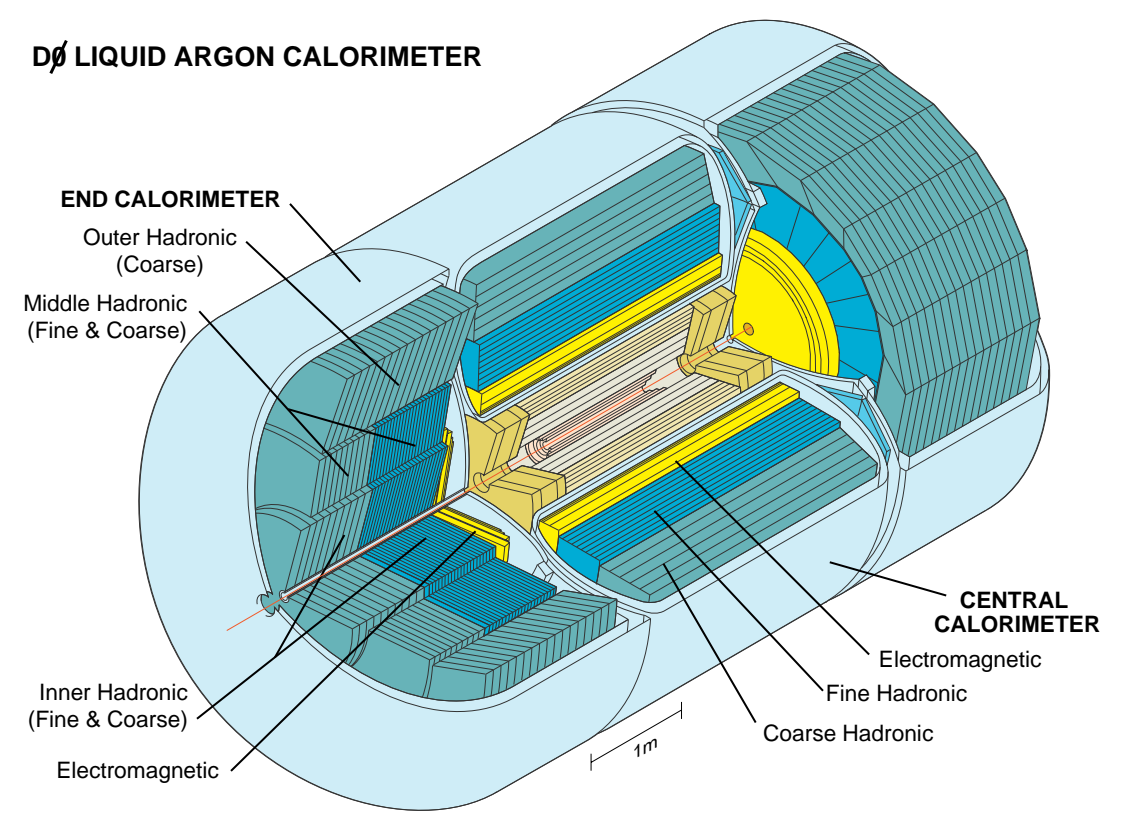

Figure 4.5. A cutaway view of the DØ Liquid Argon Calorimeter.

third read out layer covers the region of electromagnetic shower maximum and is more finely segmented than the other layers $(0.05 \times 0.05$ in $\eta \times \phi)$.

At Fermilab testbeams, the electron energy resolution for several CCEM modules were measured. The resolution, defined as $\sigma_{E} / E$, can be written as follows

$$
\left(\frac{\sigma_{E}}{E}\right)^{2}=C^{2}+\frac{S^{2}}{E}+\frac{N^{2}}{E^{2}}
$$

where $\mathrm{C}, \mathrm{S}$, and $\mathrm{N}$ are constants pertaining to the energy calibration, sampling errors and noise, respectively. The values of these constants for electrons were found to be $C=0.003 \pm 0.004, S=0.162 \pm 0.011 \mathrm{GeV}$ and $N=0.140 \mathrm{GeV}[63]$.

\section{Central Calorimeter Hadronic Modules (CCFH and $\mathrm{CCCH}$ )}

Outside the electromagnetic calorimeter lie the hadronic modules which are 6.4 interaction lengths ${ }^{3}$ deep. The EM section of the CC has 0.8 interaction lengths.

\footnotetext{
${ }^{3} \mathrm{An}$ interaction length is the mean free path between strong interaction processes.
} 


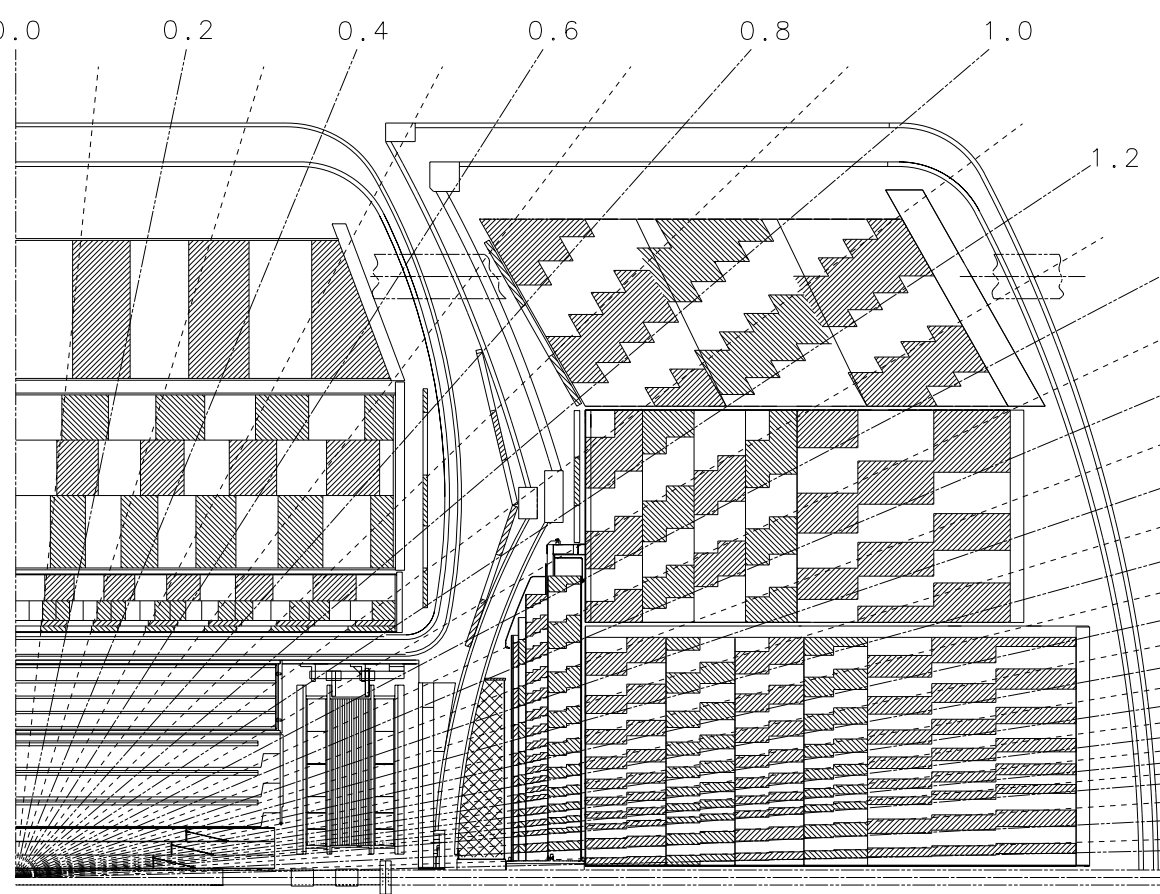

Figure 4.6. A side view of one quarter of the D $\varnothing$ detector, showing segmentation and tower definitions. Lines from the center extend along constant pseudorapidities.

The read out is segmented by radiation lengths into four segments. The modules cover the region $|\eta|<0.9$.

The first layer of hadronic modules $(\mathrm{FH})$ use uranium plates for the absorbers. The three sampling depths are at 1.3, 1.0, and 0.9 interaction lengths.

To prevent the loss of jet energy and to minimize punchthrough into the muon system, the fine hadronic calorimeters are backed by 16 coarse hadronic modules. These modules use copper as the absorber. The coarse hadronic modules have a single longitudinal segmentation of 3.2 interaction lengths. 


\section{End Calorimeters (EC)}

Two identical End Calorimeters of similar design to the Central Calorimeters were constructed to extend the $\eta$ coverage of the DØ detector. The End Calorimeters are composed of a single electromagnetic layer followed by two layers of 16 modules of hadronic calorimetry.

\section{End Calorimeters Electromagnetic Calorimeter (ECEM)}

Each module of the ECEM is a disk whose center coincides with the Tevatron beamline. The coverage of each ECEM module extends from an $|\eta|$ of 1.4 to 4.0. The longitudinal sampling layers are 0.3, 2.6, 7.9 and 9.3 radiation lengths thick. The third layer of the ECEM is more finely segmented than the other layers $(0.05 \times 0.05$ in $\eta \times \phi)$ for $|\eta|<2.6$. For $|\eta|>2.6$, the small physical size of the modules makes further segmentation difficult.

\section{EC Hadronic Calorimetry (ECIH, ECMH and ECOH)}

One Inner Hadronic (ECIH) disk module is placed behind each of the ECEM modules. The modules are disks centered about the beamline. Each ECIH module has five longitudinal sampling sections, four fine hadronic sections with 1.1 interaction lengths and a single coarse hadronic section with 4.1 interaction lengths. The fine hadronic segments use uranium as the absorber, the coarse hadronic calorimetry uses stainless steels as the absorber.

Both the outer hadronic (ECOH) and middle hadronic (ECMH) calorimeters are cyclindrical wedges arranged in rings around the beamline. The ECMH modules have four longitudinal segments at 0.9 interaction lengths with uranium absorber and one coarse hadronic section with stainless steel absorber. The longitudinal depth of the coarse hadronic section is 4.4 interaction lengths. Each of the ECOH modules has a single longitudinal segment with stainless steel absorber plates with approximately 
seven interaction lengths. The ECOH plates are inclined at an angle of 60 degrees with respect to the beamline.

\subsection{Muon Detection}

The muon detectors are well separated from the rest of the detector. Good muon identification and reasonable momentum resolutions were the primary design goals. Including the calorimeter and the muon toriod, the thickness of the detector is typically 12 interaction lengths and serves to shield the muon system from hadronic punchthrough. The muon momenta are measured from the deflection angle of muons that traverse the magnetized iron toroids. Proportional wire chambers measure position before and after muons pass through the magnetic field.

The $\mathrm{D} \varnothing$ detector can detect muons out to $|\eta| \leq 3.3$. However, in the region $|\eta|>1.7$, the backgrounds dominate and make muon identification inefficient. This region also suffers from the greatest radiation damage, making the detection systems themselves inefficient.

In this analysis, only muons with $|\eta|<1.7$ are used. In this region the muon detector is called the wide angle muon spectrometer (WAMUS), which has three superlayers of proportional drift tubes (PDTs). One plane resides in front of the magnetized iron toroid with a magnetic field of 1.9 Tesla. This layer, the A layer, has four planes of PDTs. The B and C layers, outside of the magnet and separated by approximately one meter, create a lever arm for the momentum measurement. The B and C layers each contain three layers of PDTs. Within a superlayer, adjacent layers of PDTs are offset to assist in resolving ambiguities in hit finding algorithms. The magnetic field lines and drift tube wires are oriented transverse to the direction

of the beam. The coordinate in which the muon does not bend, along the direction 


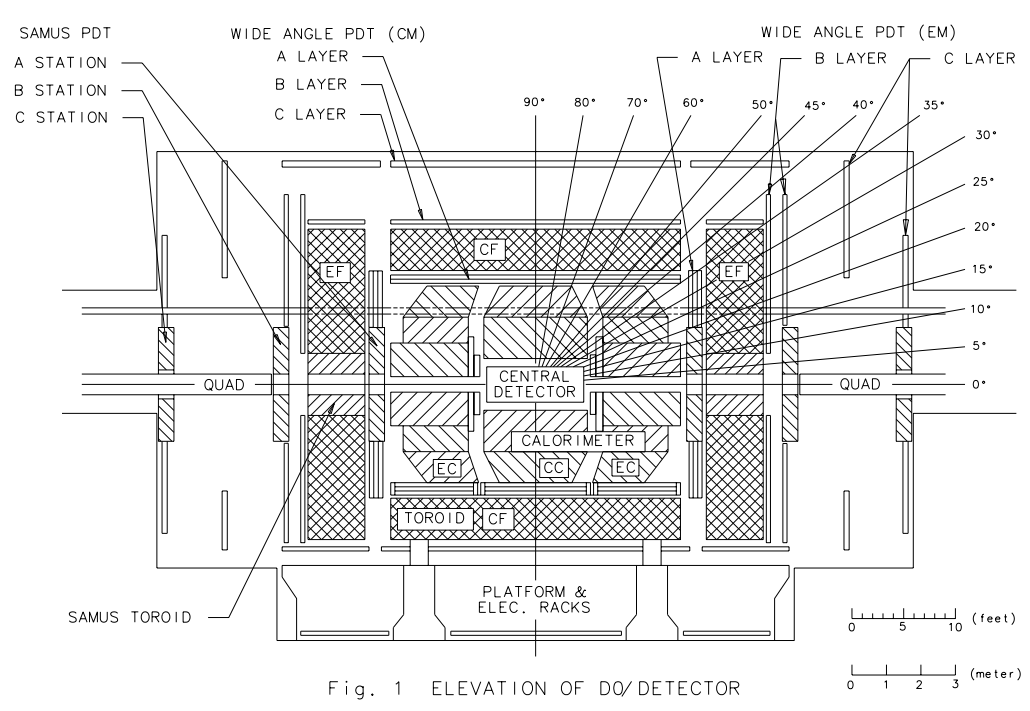

Figure 4.7. A side view of D $\varnothing$ detector, showing the entire detector muon system. of the wire, is measured less accurately and is determined by timing information indicating where the muon hit is located on the drift tube wire.

The WAMUS region extends out to $|\eta|<1.7$ over the full azimuth, with the exception of the central region, where a calorimeter support structure prevents instrumentation $\left(|\eta|>1\right.$ and $\left.255^{\circ}<\phi<315^{\circ}\right)$. The WAMUS is divided into two regions, the central iron $(\mathrm{CF})|\eta| \leq 1.0$ and the end iron region (EF) $1.0<|\eta| \leq 1.7$.

The momentum of a muon is determined by its deflection in the magnetic field of the toroid. The direction of the muon is measured before and after passing through the magnetic field. However, the measurement is limited by the multiple scattering in the material between position measurements ${ }^{4}$, knowledge of the magnetic field strength and the resolution of the position measurements. The resolution of muon momentum is approximately Gaussian in $1 / p$ and given by

$$
\sigma(1 / p)=0.18(p-2) / p^{2} \oplus 0.008
$$

\footnotetext{
${ }^{4}$ The muon must pass through the iron toroid, which can cause multiple scattering.
} 
when the momentum is in the units $\mathrm{GeV} / \mathrm{c}[64] .^{5}$

The small angle muon system (SAMUS) detector extends the coverage out to $|\eta|<3.3$, but the high hit occupancy of the SAMUS detector forces the use of smaller drift tubes than in the central region. Because of the high hit occupancy, the muon information from the SAMUS detector has not proved useful.

\section{$4.7 \quad$ Triggers}

Owing to the high rate of interactions, up to $200 \mathrm{kHz}$, and the rate at which events can be written to a magnetic tape for storage, typically $2 \mathrm{~Hz}$, it is necessary to filter out uninteresting events. The $\mathrm{D} \varnothing$ online read out has four levels of filtering to reduce the event rate. At level 0, typical rates are $150 \mathrm{kHz}$. Level 1 and 1.5 reduce the rate to $150 \mathrm{~Hz}$ for further filtering by level 2. The level 2 output rate is $2 \mathrm{~Hz}$. Nearly half of all interactions at DØ are uninteresting, i.e. elastic scattering events. An in depth discussion of the DØ trigger system can be found in [65-67]

There are three components to the $D \varnothing$ trigger system. The first two levels which use coarse and limited event information (level 0 and level 1) are hardware triggers. The final level, level 2, is a software filter which has access to the complete event record.

\section{Level 0}

The level 0 trigger is a set of hodoscope counters at either end of the calorimeter. The hodoscope counter can distinguish between beam-gas interactions and beambeam interactions. A level 0 trigger indicates that a beam-beam interaction has occurred.

\footnotetext{
${ }^{5}$ The $\oplus$ means to add the quantities in quadrature.
} 
The level 0 trigger has only rough vertex information available from the hodoscope counters. The trigger is more than $99 \%$ effective at identifying events from nondiffractive inelastic collisions.

\section{Level 1 and Level 1.5}

The level 1 trigger is a programmable hardware trigger, which can make a decision based on coarse calorimeter information and muon track information. Usually, the level 1 trigger is fired ${ }^{6}$ after a collision has been detected by level 0 .

When a collision has occurred, the detector information is read out and the analog signals proceed along two different paths. One path, the trigger pickoff path is used to make a decision at level 1 . The second precision read out path keeps the full detector information so that the event can proceed to level 2 , if the event is deemed interesting. The trigger pickoff has coarse calorimeter cell information of size $0.2 \times$ 0.2 in $\eta \times \phi$. This path does not have access to all of the longitudinal segments of the calorimeter. The level 1 trigger estimates the calorimeter transverse energy by using a lookup table and vertex information from level 0 . Electromagnetic and hadronic cells are summed separately.

A trigger menu, a downloaded specification of the characteristics of interesting events, is compared against the event characteristics of level 1 . The trigger menu uses rudimentary information, such as the number of trigger towers in the calorimeter above a threshold, to determine if an event has passed a trigger. There are 32 distinct triggers which can be programmed at level 1. If an event has been found to satisfy a trigger, then the event is digitized and sent to the level 2 processor farm. The decision must be made before the next beam crossing and with enough time for the

\footnotetext{
${ }^{6}$ A trigger "fires" for an event that passes the trigger requirements.
} 
detector electronics to reset. The level 1 trigger makes a decision within $2.2 \mu$ sec. The beam crossing time is $3.5 \mu$ sec.

For muons, the level 1 trigger determines the approximate centroid of each WAMUS chamber hit. A centroid is the muon PDT cell which most likely contains the location of the track. If a hit is found in the B or C layer, a match is looked for in the A layer. If a match is found, a signal is sent to the trigger framework. The signal notifies level 1.5 of the centroid list for verification. The centroids are used to determine if the muon detected passes the programmed $p_{T}$ requirements. For a muon momentum measurement $1 \%$ dead time was necessary, since it required $28 \mu$ sec.

If an event is found to be interesting, it can be completely digitized and read out at any time during the level 1 or 1.5 trigger. If the event is not read out, the hardware is reset and the event data are lost. Typically, the rate into level 2 is about $150 \mathrm{~Hz}$.

\section{Level 2}

At level 2, the final decision is made on whether to keep or reject. Once level 1 has accepted the event, and after it has been digitized, the event is sent to a farm of 48 MicroVAX 4000/60 computers which make up the level 2 filter system. Although the data are complete, only a partial reconstruction of physics objects is performed.

The level 2 farm has up to 128 software filters each associated with a trigger from level 1. If an event passes one of the filters, it is sent to the DØ Host computer and written to magnetic tape.

\subsection{Data Acquisition}

Data are acquired when a trigger has fired. As the level 1 trigger is passed, the Supervisor $^{7}$ is notified and subsequently notifies the data acquisition cards to begin

\footnotetext{
${ }^{7} \mathrm{~A}$ Supervisor is a computer whose purpose is to control specified aspects of data acquisition.
} 
event digitization. The acquisition cards require $1 \mathrm{msec}$ for digitization. The data are then read out on eight unidirectional data cables, each of which can transfer 40 Mbyte/s.

The data cables are connected to multi-port memory boards (MPMs) on each of the level 2 nodes. The Supervisor selects a node to receive and process the event.

\subsection{Modeling the Detector Response}

Many aspects of the D $\varnothing$ detector have been modeled using GEANT [68]. The GEANT program simulates the propagation of final state particles through the detector. But since the program is too CPU-intensive to simulate trajectories and energy showers in detail a technique called the shower library was developed by DØ. GEANT is used to simulate, in detail, a large sample of electrons, hadrons and muons and the results stored in a random access library [69]. When events are simulated, an appropriate shower from the library is chosen and used to simulate event showers.

The muon and trigger responses are not accurately accounted for by the D $\varnothing$ GEANT simulation. The simulation of muons is improved with a post-GEANT routine called MUSMEAR [70]. The trigger efficiency is modeled using trigger turn-on curves obtained from data samples. 


\section{CHAPTER 5}

\section{PARTICLE RECONSTRUCTION AND IDENTIFICATION}

\subsection{Particle Reconstruction}

The $D \emptyset$ detector provides a wealth of information about the high energy events that have been recorded. The event is stored in a ZEBRA [71] format with banks that correspond to the raw read out of individual components of the DØ detector, as well as the physics objects reconstructed from the event.

The D $\varnothing$ reconstruction program finds jets, electrons, photons, missing transverse energy and muons, using algorithms that convert the electronic read out data into the physical objects of interest.

\section{DØRECO: The Reconstruction Program}

The DØRECO program is the software that reconstructs physics objects from the detector signals. The program first converts detector signals to hits. For example, in the vertex detector or the muon detector, a hit is a current pulse on a wire. For the calorimeter, a hit is the amount of energy deposited in a particular cell.

\section{Vertex Reconstruction}

In order to determine most other quantities accurately, it is crucial to measure the z-position of the vertex accurately. The z-position of the vertex (the z-vertex) varies 
from the nominal position of the collision point about $\pm 30 \mathrm{~cm}^{1}$. Consequently, the z-vertex varies from event to event. The DØRECO program is able to reconstruct multiple vertices, arising from multiple collisions in a single beam crossing.

The first step in reconstructing a collision vertex is to find all of the charged particle tracks in the three tracking systems: VTX, CDC and FDC. Each charged track is extrapolated back to the beam position at $(\mathrm{x}, \mathrm{y})=(0,0)^{2}$ and the $\mathrm{z}$-position of the track is stored. Next, the z-positions are clustered. Each cluster is then fitted to a Gaussian distribution and the mean z-position extracted.

The vertex which has the most tracks originating from it is then labeled as the primary vertex. However, if the algorithm cannot find a vertex, then the vertex is set to the origin of the coordinate system and all subsequent quantities are calculated with respect to the origin.

A vertex position can be biased if there are many tracks in an event. The vertex algorithm does not weight tracks which are directed toward large energy deposits. Events which have large track multiplicities can have wide distributions of z-positions which can shift the mean from the true vertex. In such events, there are frequently two vertices. The primary vertex may not be the vertex associated with the objects with the largest deposits of energy.

The vertex is very important for determining the muon momentum accurately, because the bend angle of the muon is crucial for good muon measurement. The vertex position measurement can also be used to reject cosmic ray muons.

\section{Jet Reconstruction}

Over the years, a number of algorithms have been developed to reconstruct jets. One of the algorithms, called the Durham algorithm is a nearest neighbor algorithm,

\footnotetext{
${ }^{1}$ This is the longitudinal extent of a Tevatron particle bunch.

${ }^{2}$ The variance in the $\mathrm{x}$ and $\mathrm{y}$ positions of the beam is very small, typically, $30 \mu \mathrm{m}$.
} 
where a jet extends until there is no more energy in the neighboring cells above a threshold. D $\varnothing$ uses an iterative cone algorithm for jets. In the iterative cone algorithm, a jet has a predetermined extent in $\eta-\phi$ space and begins with a seed tower. A tower is an extended deposit of hadronic energy in calorimeter cells. The event vertex is used to calculate the $E_{T}, \eta$, and $\phi$ of calorimeter hits before the algorithm is executed.

The cone algorithm begins with an $E_{T}$ ordered list of seed towers. A tower is put into the seed list if its $E_{T}$ is greater than $1 \mathrm{GeV}$. Preclusters are formed from the towers. The precluster position $(\eta, \phi)$ is determined by the seed tower. All towers on the list and that fall within the cone, centered on the seed tower, are added to the jet and the centroid of the jet is re-calculated using the $(\eta, \phi)$ position of the towers and weighted by the $E_{T}$ of each tower. Each of the towers found around the first seed are removed from the list and the next highest $E_{T}$ seed is used to create the next jet. This process is repeated until there are no seed towers remaining. The preclustering reduces the number of seeds that must be formed in the clustering stage of the algorithm.

We define the energy vector $\vec{E}_{i}$, associated with a calorimeter tower $i$ as

$$
\vec{E}_{i}=\hat{n} E_{i}
$$

where $\hat{n}$ is the unit vector in the direction of the tower and $E_{i}$ is the sum of the energies of all cells in the tower $i$. Similarly, we define the transverse energy associated with a tower $i$ as $E_{T}^{i}$. This quantity is the vector sum of all cells in tower $i$ projected onto the $\mathrm{x}-\mathrm{y}$ plane,

$$
E_{T}^{i}=\sqrt{\left(E_{x}^{i}\right)^{2}+\left(E_{y}^{i}\right)^{2}}
$$

$E_{T}^{i}$, the transverse energy of a calorimeter tower is the starting point for finding jets at $\mathrm{D} \varnothing$. 
Jet clustering begins by placing all of the preclusters in descending ordering of $E_{T}$. The $\eta$ - $\phi$ positions of the highest $E_{T}$ precluster is used as the center of a cone of given radius $R$ and all the towers within that cone are summed. The $E_{T}$ weighted centroid is calculated using:

$$
\begin{gathered}
E_{T}^{j e t}=\sum_{i=1}^{<\text {all towers }>} E_{T}^{i}, \\
\eta^{j e t}=\frac{\sum_{i=1}^{<\text {all towers }>} E_{T}^{i} \times \eta_{i}}{E_{T}^{j e t}}, \text { and } \\
\phi^{j e t}=\frac{\sum_{i=1}^{<\text {all towers }>} E_{T}^{i} \times \phi_{i}}{E_{T}^{j e t}} .
\end{gathered}
$$

The new position of the jet is then compared with its previous position that of the precluster. If the difference between the previous and new positions is greater than 0.001 in $\eta$ - $\phi$, the process is repeated until the difference between the current position and the previous position is less than 0.001 in $\eta-\phi$. To prevent the program from oscillating about two bi-stable points, a maximum of 50 iterations is imposed on the algorithm.

Finally, the angles for the jet are recalculated using a different definition from the previous definition in Eqs. 5.4, 5.5. The new definitions for $\eta$ and $\phi$ are given in Eqs. 5.6 - 5.8, the quantities $E_{x}^{i}, E_{y}^{i}$, and $E_{z}^{i}$ are the three components for the energy vector of each tower cell. If the $E_{T}$ is less than $8 \mathrm{GeV}$, the jet is rejected by the reconstruction program.

$$
\phi=\arctan \left(\frac{\sum_{i=1}^{<\text {all cells }>} E_{y}^{i}}{\sum_{i=1}^{<\text {all cells }>} E_{x}^{i}}\right)
$$




$$
\begin{gathered}
\theta=\arctan \left(\frac{\sum_{i=1}^{<\text {all cells }>} E_{z}^{i}}{\sqrt{\sum_{i=1}^{<\text {all cells }>} E_{x}^{i 2}+\sum_{i=1}^{<\text {all cells }>} E_{y}^{i 2}+\sum_{i=1}^{<\text {all cells }>} E_{z}^{i 2}}}\right) \\
\eta=-\ln \left(\tan \frac{\theta}{2}\right)
\end{gathered}
$$

The clustering process begins again with the next highest precluster that is not within the jet cone of the previously found jet. The process continues until there are no preclusters left. The DØRECO program reconstructs jets with a variety of cone sizes: $0.3,0.5,0.7$ and 1.0.

With the jet cone algorithm, jets may share energy and a decision must be made whether to merge or split jets. Beginning with the second jet, each new jet is checked to see if it shares energy with other jets. If shared towers are found, the jet axes are compared. In some cases due to round-off errors a 'new' jet is simply a jet already found. This is true if the distance between the two jets axes is less than 0.01 in $\eta-\phi$ space. In this case the 'new' jet is dropped.

In the case that the two jets are not the same, a decision is made to determine if the jet should be split or merged, based upon the fraction $f_{S M}$, defined by

$$
f_{S M}=\frac{E_{T}^{\text {shared }}}{E_{T}^{\text {min }}} .
$$

In Eq. 5.9, $E_{T}^{\text {shared }}$ is the transverse energy of the common towers between the two jets and $E_{T}^{\text {min }}$ is the smaller transverse energy of the two jets. If $f_{S M} \leq 0.5$ then the two jets are split and the cells (not towers) are split among the two jets according to which jet is closer to the cell. If $f_{S M}>0.5$, the two jets are merged into a single jet and all towers are assigned to it.

The quality of a jet is determined from quantities dependent on the pattern of energy deposits in the jets. For example, it is possible for a jet to be mis-identified as an electron if most of its energy lies within the electromagnetic calorimeter. 


\section{Jet Energy Calibration}

The DØ program CAFIX $^{3}$ is designed to correct the jet energies for calorimeter effects [72].

The CAFIX program corrects for the following detector effects:

- Hadronic response $R_{h}$. $R_{h}$ is a measure of how well the calorimeter responds to hadronic energy deposits. $R_{h}$ may be different from unity because of an extended hadronic shower, regions of no or poor instrumentation, a non-linear response for particles below $10 \mathrm{GeV}$, and differences between testbeam modules and those used in DØ.

- Energy due to the underlying event.

- Noise due to electronics and fission of the uranium nuclei.

- Energy which falls outside of the cone of the jet.

Once these factors have been accounted for, the jet energy $E_{j e t}^{c o r r}$ is given by

$$
E_{j e t}^{c o r r}=\frac{E_{j e t}^{R E C O}-O}{(1-S) R_{h}} .
$$

In Eq. 5.10, $E_{j e t}^{R E C O}$ is the energy of the reconstructed jet from DØRECO, $O$ is the energy from the underlying event and noise, $S$ is the correction factor for out-of-cone showering.

\section{Missing Transverse Energy - The Neutrino}

Neutrinos rarely interact with matter and consequently cannot be detected directly by the DØ detector. However, the neutrinos carry away energy which is

\footnotetext{
${ }^{3}$ CAFIX typically runs as a separate routine after the reconstruction is finished.
} 
"unseen" by the calorimeter and other energy detection systems. Although the zmomentum of the incoming partons is unknown, the $\mathrm{x}$-momentum and y-momentum of the incoming partons sum approximately to zero.

Since the $x$ and $y$ components of momentum of the initial partons is approximately zero, there must be a balance in the transverse momentum of an event. If the $\mathrm{x}$ and y components of the energy of the final state objects do not sum to zero, then there is missing transverse energy in the event, $\mathbb{E}_{T}$. DØRECO calculates the missing transverse momentum three times. The first calculation is a cell by cell vector sum of the transverse energies in the calorimeter, the result is the negative of the $\mathbb{E}_{T}$ vector. The second calculation corrects the $\mathbb{E}_{T}$ in the Inter Cryostat Detector (ICD). The third calculation corrects the $\mathscr{E}_{T}$ for any muons which are found in the event.

The $\mathbb{E}_{T}$ vector is defined as

$$
\begin{aligned}
& E_{x}=-\sum_{\text {cells } i} E_{x}^{i}, \\
& E_{y}=-\sum_{\text {cells } i} E_{y}^{i} .
\end{aligned}
$$

\section{Muon Reconstruction}

To reconstruct muons, the muon detection system records data from a series of wires indicating the presence of a hit in a given drift cell, along with the hit, analog signals which record the drift time and the charges on the inner and outer segments of cathode pads. The drift time is the difference in time between the signals read out at each end of the sense wire.

The reconstruction process begins by locating the spatial location of hits. The hits are joined into two straight segments, one segment for the A-layer cells and the second from hits in the $\mathrm{B}$ and $\mathrm{C}$ layers. There is a left-right ambiguity in the muon system hit detection which is removed by using a $\chi^{2}$ from a linear least-squares fit to the "left" and "right" hits of the segments. The $\chi^{2}$ fit of the segments also removes 
any ambiguity about the z-position of the hits. B and C layer segments are required to have four of six possible hits. A-layer segments are required to have two of four possible hits. To reject cosmic ray muons, all segments are required to point to within $5 \mathrm{~m}$ of the center of the detector.

The two segments are then connected. The B/C layer segments are extended to the mid-plane of the toroid. The A-layer segment that most closely matches the B/C segment is then "connected" to the track of the candidate muon. If no A-segment exists for the B/C layer track, then the pre-toroid muon direction is assumed to be the line between the $\mathrm{B} / \mathrm{C}$ segment and the primary interaction vertex. It is also possible to have a muon candidate with no B/C layer tracks. Such candidates are called A-stubs and are rejected in the selection process. The A-stub candidates have no muon momentum information.

To first order, the muon momentum measurement is proportional to the angle between the $\mathrm{A}$ and $\mathrm{B} / \mathrm{C}$ segments of the track, with corrections for energy lost by the muon as it traverses the calorimeter. A momentum measurement becomes much more precise if a global fit is performed. The global fit makes use of 16 input variables:

- The $\mathrm{x}$ and $\mathrm{y}$ coordinates of the event vertex.

- The slope and intercept of the Central Drift Chamber (CDC) track in the $r-z$ and $r-\phi$ views.

- Two angles representing the mismatch of the CDC track and calorimeter track directions.

- The slope and intercept for the A and B/C segments for the $r-z$ and $r-\phi$ views.

The result of the fit is seven parameters: four for the CDC track, two parameters, which represent multiple scattering in the detector, and the muon momentum. 
In order to provide rejection against backgrounds such as cosmic rays, random noise hits in the muon system and hadronic punchthrough in the gap region between the central and end toroids, several variables are used to indicate the goodness of fit:

- An integer, IFW4 indicating the goodness of fit. An IFW4 value of 0 indicates a perfect global fit. Muons with an IFW4 of 1 have one track flaw in the fit. For example, a muon track which does not point to the primary vertex may have a single track flaw. All muon candidates in this analysis must have a value for IFW4 of 0 or 1 . IFW4 will be referred to as Muon Track Badness.

- MTC (muons in the calorimeter). Calorimeter information can be used to verify that a minimum ionizing particle has passed through the calorimeter. Two quantities are especially useful: hfrac, the number of hits in the hadronic layers divided by the maximum number of hits possible along the candidate track and efrac, the amount of energy in a $3 \times 3$ cell region about the candidate muon track of the last layer of the electromagnetic calorimeter.

- The quantity $\int \vec{B} \cdot \overrightarrow{d l}$ measures how much magnetic field the muon passes through. This quantity is used to reject tracks which pass through the inter-toroid crack. Punchthrough is a significant background here.

- Track 3D impact parameter. Tracks which do not originate near the collision point are likely to be cosmic rays. This parameter is used to reject such tracks. The 3D impact parameter is determined from the distance between the muon track in the bend view (post-toroid) and in the no-bend view (pre-toroid).

- Scintillator Timing. Some, though not all, of the muon detectors have scintillator timing information available for muon candidates. The timing is used for cosmic ray rejection. Scintillator timing was not used in this analysis. 
The muon system suffered degradation over time. Polymer deposits on anode wires decreased the muon detection efficiency. Eventually, the problem became significant enough to require cleaning of the chamber wires - a process referred to as "zapping". For muons, the data can be divided into three run periods: Run IA, Run IB pre-zap and Run IB/C post-zap. During Run IA, MTC track verification was not stored and a simpler quantity is used for calorimeter verification. Run IA muon data also had problems with muons between $90<\phi<110$, due to Main Ring punchthrough.

\subsection{Particle Identification}

The algorithms and selection criteria used to identify isolated muons, tagging muons, jets and neutrinos are discussed below.

\subsubsection{Muon ID}

In this analysis, we will apply two sets of requirements for muons and distinguish them as tagging or isolated muons. An isolated high $p_{T}$ muon dominantly comes from the decay $W \rightarrow \mu \nu$. A tagging muon will typically be non-isolated (i.e., embedded in a jet), have softer $p_{T}$ and mostly arises from a heavy flavor decay as in $b \rightarrow X \mu \nu$.

The efficiencies for the muon identification are discussed in order to display the losses due to the different muon identification requirements. However, these efficiencies will not be used because the muon system is sufficiently well modeled by the muon simulation program MUSMEAR. However, there is a slight disagreement between data and Monte Carlo muons which is corrected for by the eye-scan "efficiencies" discussed below.

Owing to improvements in muon identification and the excellent calorimetry available with the $\mathrm{D} \varnothing$ detector, it was realized that muons could be identified or verified with the DØ calorimeter. A tool was developed for Run IB/IC, known as 
the Muon Tracks in Calorimeter (MTC) Package [73]. During Run IA, the MTC package was not available, but calorimeter information was available in the form of minimum ionizing particle (MIP) energy deposits and energy deposits in its nearest neighbor. The differences between Run IA and IB/IC for muon identification are due to the improvements available with the MTC package.

\section{Isolated Muon ID}

To reduce the size of the background data sample as much as possible only events with isolated muons were kept, as described in Section 6.2. For the final signal sample, the muon selection criteria for isolated muons in Run I are

1. Muon Quadrant $\leq 4(\leq 12)$ for runs $<89,000(\geq 89,000)$

2. For CF muons $(1 \leq$ Iquad $\leq 4)$, Muon Track Badness $=0$ or 1

3. For postzap EF muons $(5 \leq$ Iquad $\leq 12)$, Muon Track Badness $=0$ (otherwise, reject EF muons)

4. No A-stubs

5. IA: Calorimeter MIP deposit $>1 \mathrm{GeV}$ and Nearest Neighbor deposit $>1 \mathrm{GeV}$

IB $/ \mathrm{IC}:$ frac $=1.0$ or $($ frac $>0.7$ and efrac $>0$. $)$

6. 3D Impact parameter cut $\sqrt{I P_{B V}^{2}+I P_{N B}^{2}} \leq 20.0 \mathrm{~cm}$

7. $\int \vec{B} \cdot \overrightarrow{d l} \geq 0.6 \mathrm{~T} \cdot \mathrm{m}$

8. $p_{T} \geq 20 \mathrm{GeV} / \mathrm{c}$

9. Isolation $\Delta R_{\mu, j e t}>0.5$ 
Table 5.1. Muon efficiencies for the definitions used in this analysis. The muon represented here are from the decay of the W. A good muon candidate passes both the Muon Track Badness cuts and calorimeter verification. PMUO is the bank that contains information about the reconstructed muon.

\begin{tabular}{|l|r|r|}
\hline & \multicolumn{2}{|c|}{ Efficiency (\%) } \\
Requirement & $\mathrm{CF}$ & $\mathrm{EF}$ \\
\hline w/good PMUO & $\equiv 100.0$ & $\equiv 100.0$ \\
w/good PMUO, $p_{T}>20$ & $84.1 \pm 0.7$ & $74.1 \pm 1.5$ \\
Remaining ID cuts & $68.4 \pm 0.9$ & $67.4 \pm 1.6$ \\
Isolation & $64.3 \pm 0.9$ & $63.6 \pm 1.6$ \\
\hline
\end{tabular}

The first cut selects muons where our instrumentation is reliable and well understood. The Muon Track Badness cuts, the second and third criteria above, reject muons which do not have good quality tracks in the muon detection system. The fourth cut rejects muons which do not make it through the first section of the iron toroid and therefore have poor momentum measurement. The fifth criterion is the calorimeter verification of a muon [73]. This criterion along with the impact parameter cuts serve to reject cosmic rays, combinatoric fakes (muon candidates due to accidentally aligned noise hits) and mis-reconstructed muons. The quantities $I P_{B V}$ and $I P_{N B}$ are the $2 \mathrm{D}$ distances of closest approach between the muon and the vertex in the bend and non-bend views, respectively. The $\int \vec{B} \cdot \overrightarrow{d l}$ cut assures that the muon has seen a large enough magnetic flux to afford a good momentum measurement. Finally, the $p_{T}$ and isolation cuts describe muons typical of those coming from the decay of a $W$-boson.

The muon reconstruction efficiency is expressed in terms of three quantities. A good muon candidate requires the MTC (Muon Track in the Calorimeter) verification and Muon Track Badness requirements. This requirement has an efficiency of $67.2 \pm$ 
$0.1 \%$ for any muon in the CF and an efficiency of $75.5 \pm 1.3 \%$ for any muon in the EF region. The next requirement is that of high $p_{T}$. The remaining ID cuts, except isolation, are on the amount of magnetic flux seen and the impact parameter cuts. Finally, as the jet multiplicity increases, there is a greater chance that the muon will not be isolated due to accidental overlaps with jets. The efficiencies are summarized in Table 5.1.

\subsubsection{Tagging Muon ID}

The muon selection cuts for tagging muons in Run I are

1. Muon Quadrant $\leq 4(\leq 12)$ for runs $<89,000(\geq 89,000)$

2. For CF muons $(1 \leq$ Iquad $\leq 4)$, Muon Track Badness $=0$ or 1

3. For postzap EF muons $(5 \leq$ Iquad $\leq 12)$, Muon Track Badness $=0$ (otherwise, reject EF muons)

4. No A-stubs

5. IA: Calorimeter MIP deposit $>1 \mathrm{GeV}$ and Nearest Neighbor deposit $>1 \mathrm{GeV}$

$\mathrm{IB} / \mathrm{IC}:$ frac $=1.0$ or $($ frac $>0.7$ and efrac $>0$. $)$

6. $p_{T} \geq 4 \mathrm{GeV} / \mathrm{c}$

7. Isolation $\Delta R_{\mu, j e t}<0.5$

The requirements for the tagging muons are similar to those of isolated muons. The differences are the absence of the high $p_{T}$ requirement and the requirement that tagging muons be non-isolated. The minimum $p_{T}$ of $4 \mathrm{GeV} / \mathrm{c}$ is approximately the minimum $p_{T}$ for a muon to penetrate the calorimeter and the iron toroid of the muon detection system. 
Table 5.2. Muon efficiencies for detection of a tagging muon from a b-jet. Both CF and EF muons are represented here. Recall that no events with EF muons are used before run 89000 .

\begin{tabular}{|l|r|}
\hline Detector Region & Efficiency (\%) \\
\hline $\mathrm{CF}$ & $94.7 \pm 0.4$ \\
$\mathrm{EF}$ & $88.5 \pm 3.3$ \\
\hline
\end{tabular}

The reconstruction efficiencies are the same as those in the $t \bar{t}$ analysis for tagging muons. The top group has determined the efficiency for tagging muons using data. Typically, $\mathrm{Z} \rightarrow \mu^{+} \mu^{-}$events are used to determine the efficiency for a set of muon cuts. One of the muons is required to pass the tight set of cuts and the second muon is used to determine the efficiency of a given set of cuts.

However, for muons in some $\eta$ ranges $\mathrm{Z} \rightarrow \mu^{+} \mu^{-}$statistics are limited. This is the case in the EF region. The muon efficiency for EF tagging muons is determined from a multi-jet trigger. One then assumes that all real muons are associated with jets and are thus tagging muons. Any isolated muons are assumed to be fake muons due to combinatoric backgrounds. This is reasonable because the heavy flavor cross sections are orders of magnitude larger than the $W+2$ jet cross section $[75,96]$.

The efficiencies are summarized, for tagging muons in the CF and EF, in Table 5.2. The references for these results are [74] and [75].

\subsubsection{Eye-Scan Efficiencies}

The Monte Carlo muons used in this analysis have been processed with MUSMEAR, which models the detector effects for muons well. However, there is an overall correction necessary to make the Monte Carlo precisely model the data. Although the overall factors are referred to as efficiencies, they are actually scale factors that correct 
for over-estimation of muon efficiencies within D $\varnothing$ by the Monte Carlo modeling of muons plus MUSMEAR.

The correction was determined by scanning both data and Monte Carlo muon events by eye and then comparing the efficiencies of data and Monte Carlo scans. The results are described below.

The eye-scan "efficiency" for CF muons has been determined to be $94.1 \pm 1.8 \%$ for all run ranges. For EF muons, this efficiency is $91.1 \pm 1.9 \%$ for all run ranges [98].

We use the eye-scan efficiencies for high $p_{T}$ isolated and tagging muons. In addition to the eye-scan efficiencies, for muons detected before "zapping" the muon chambers, there was an inefficiency for muons in $\phi, 80<\phi<120$ about the Main Ring. The region is known as the $\phi$-hole. The $\phi$-hole efficiency for Run IB prezap is $90 \pm 5 \%$ and for Run IA, $95 \pm 5 \%$.

\subsubsection{Jets and Missing Transverse Energy}

The jets in this study were reconstructed using an iterative cone algorithm, with a cone size of $\Delta \mathrm{R}=0.5$ in $\eta-\phi$ space. The jet energies were corrected, as a function of $\eta$ for both data and MC events, using the jet correction program CAFIX version 5.1. A jet is defined as a calorimeter object having $p_{T}>15 \mathrm{GeV} / \mathrm{c}$ and $|\eta|<2.5$.

The missing transverse energy, $\mathbb{E}_{T}$, is determined by requiring transverse energy balance in the calorimeter. We use two different definitions of $\mathbb{E}_{T}$ :

- $\not_{T}^{c a l}$ : the calorimeter missing $E_{T}$, (in the bank PNUT4)

- $\mathbb{E}_{T}$ : the muon corrected missing $E_{T}$, calculated from the value in PNUT4 and the transverse momenta of good isolated muons.

An algorithm, called AIDA, was used to reject hot cells due to random energy discharges in the events. AIDA finds hot cells by analyzing the pattern of longitudinal energy deposition within the calorimeter. Frequently, AIDA will classify a cell as hot 
although the cell is within a jet. Falsely suppressed hot cells give rise to additional calorimeter $\mathbb{E}_{T}$. This is corrected for. In addition, if a hot cell (documented in a CAID bank) lies within $\Delta R$ of 0.25 of a jet, then the hot cell is added vectorially to the calorimeter $\mathscr{E}_{T}$. The particular value of $\Delta R$ chosen is based upon where most hot cells are located in $\eta-\phi$ space of a jet [99]. We shall refer to $\mathbb{E}_{T}$ as the corrected missing transverse energy. 


\section{CHAPTER 6}

\section{MUON+JETS WITH $\mu$-TAG ANALYSIS}

\subsection{Analysis Synopsis}

The analysis presented here is accomplished by first recognizing a series of cuts that optimize the signals while minimizing the backgrounds. This is accomplished by systematically studying the single top kinematic quantities and at the same time selecting the variables that best select single top events. Next, the backgrounds are calculated and then the selection applied to the data. The goal at the beginning of this analysis was to measure a cross section for single top. Cross sections are measured by counting data events and then determining the backgrounds. Once properly normalized to the data, the number of background events $(B)$ are subtracted from the number of data events $(N)$. If an excess number of events is found, a cross section $(\sigma)$ can be determined via,

$$
\sigma=\frac{N-B}{\epsilon \int L d t},
$$

where $\epsilon$ is the total efficiency for finding single top events and $\int L d t$ is the integrated luminosity. If an excess is not found, a cross section limit is appropriate.

One finds that the single top events are very similar to several other types of events, among them, $W+$ jets physics processes and $t \bar{t}$ events. In addition to these events, a QCD process can fake single top events. It is very difficult to distinguish single top events from these other processes. In order to drastically 
reduce the backgrounds (thereby increasing the signal to background ratio), one can use $b$-tagging.

Heavy flavor or b-tagging most likely occurs when a b-quark decays semileptonically. Since single top events are expected to have at least two $b$-quarks in each event, the signal to background ratio is enhanced. The $b$-quark decay can be "tagged" by searching for events which have a muon or electron inside the cone of a jet. While the electron "tagging" is currently under extensive study [87], the muon "tagging" is well studied and methods have been devised to calculate the signal and backgrounds. The muon within the jet is said to be a "tagging" muon and the jet in which the muon is found is called a "tagged" jet.

By applying a "tag" the process of background calculations is made more complex. In order to calculate backgrounds, a tagrate function is developed and used to predict the backgrounds. A tagrate function parameterizes the probability of a jet to produce a "tagging" muon. This technique uses jet properties to parameterize this probability.

Once the backgrounds are calculated, the total background in $85.6 \mathrm{pb}^{-1}$ is found to be $8.1 \pm 1.3$. When the same selection criteria is applied to the data, one finds 8 data events. Since no excess of events is found, single top is not observed. Instead, cross section limits are placed on the single top production cross section. The details of the above calculation will now be discussed.

\subsection{Pre-selection of data}

The $t \bar{t} \rightarrow \mu+$ jets group performed a data preselection of the Run I data with a preselection that is very appropriate for single top events. The $\mu+$ jets preselection created two separate classes of events, one for signal and one for background. The requirements for the signal class were: one or more loose isolated muons and one or 
more (CAFIX) corrected jets with $|\eta|<2.5$ and $E_{T}>10$. The isolation requirement was $\Delta R(\mu$, nearest jet $)>0.5$.

The background class required one or more loose muons and the same jet selection as the signal stream. There was no isolation requirement for the pre-selected background stream. The background class is used later to estimate the QCD multi-jet background in Section 6.5.1.

A loose muon is one which passes the following requirements:

- Muon Track Badness (IFW4) $\leq 1$

- Central and Forward muons

- Calorimeter Confirmation

- no A-stub (muons that do not have hits in the B or C layers)

- $p_{T}>15 \mathrm{GeV} / \mathrm{c}$

- or -

- Muon Track Badness (IFW4) $\leq 1$

- Central and Forward muons

- no A-stub

- $p_{T}>15 \mathrm{GeV} / \mathrm{c}$ and when using a differnt vertex $p_{T}{ }^{\prime}>15 \mathrm{GeV} / \mathrm{c}$.

- $\chi^{2}$ (Track Match Significance) $>10$ or no track match

The first set of requirements is the "minimal" set for a muon; the second set is designed to recover muons lost because of an incorrect choice of vertex. If the vertex is wrong, then we expect no track match and/or a large value of $\chi^{2}$, the track match 
Table 6.1. Events pre-selected for Run I. Only the Run IB background sample was used, the number of pre-selected events in this sample was 334,906.

\begin{tabular}{|l|r|}
\hline \multicolumn{2}{|c|}{ Events in pre-selected signal samples } \\
\hline \hline Run IA & 2,441 \\
Run IB & 189,772 \\
Run IC & 8,384 \\
\hline
\end{tabular}

significance. The track match significance is the result of a least-squares fit of the muon track considering the $r-z$ and the $r-\phi$ information separately. Owing to a left-right ambiguity in the muon detection hardware, a track fit is required to resolve the ambiguity. A value of $\chi^{2}>10$ is a poorly fit muon track and an indication that the wrong vertex may have been used. The muon word IFW4 is an indicator of muon "goodness". In simple terms, IFW4 is a count of the number of bad features of a muon track. The "cal confirm" requirement uses the D $\varnothing$ calorimeter to confirm that a muon has passed through the detector. The A-Stub veto rejects muons which do not make it through the iron toroid, and therefore do not have a good momentum measurement.

Each set of requirements allows both central $(\mathrm{CF})$ and forward (EF) muons. The CF muons are found in the region approximately $|\eta| \leq 0.95$. The EF muons extend out to $0.95<|\eta|<1.7$. (These regions are naturally defined by the layout of the quadrants of the muon system, so there could be some overlap between what is a CF muon and an EF muon when the pseudo-rapidity is used to classify a muon as CF or EF. A CF muon is found in quadrants 1-4 and an EF found is found in quadrants 5-12.)

The preselection for Run IA data was also available and the events have been reprocessed to update them with the latest versions (RECO 12.21) of the reconstruc- 
tion algorithms where possible. The selection requirements differ for muon events taken during Run IA. The quantities needed for calorimeter verification of muons were not available for Run IA data. However, rudimentary calorimeter identification was available.

\subsection{The Selection Requirements}

We begin with the data sample described in Section 6.2, with one good corrected jet and one good isolated muon. This base sample has 185,000 data events remaining after the initial selection. After reconstruction and selecting clean events with between two and five jets, exactly one good isolated muon and more than $15 \mathrm{GeV}$ of $\not_{T}^{\text {cal }}$, we have a sample of 17,055 events. The number of events surviving each selection cut is shown in Table 6.2.

For the muon+jets tagged analysis, the selection criteria are shown below.

- One isolated muon and trigger requirements (MU_JET_XXX)

- Good jets (Section 5.2.4) and Main Ring veto

- $H_{T}^{c a l} \geq 20 \mathrm{GeV}$

- $\mathbb{E}_{T} \geq 20 \mathrm{GeV}$

- $2 \leq \mathrm{N}_{\text {jets }} \leq 5$

- $E_{\text {Tjet } 1} \geq 20 \mathrm{GeV},|\eta|<2.5, E_{T j e t 2} \geq 15 \mathrm{GeV},|\eta|<2.5$

- one or more tagging muons

- $\mathrm{P}\left(\chi^{2}\right)<0.01$, with $\chi^{2}$ from a $Z \rightarrow \mu \mu$ kinematic fitter

- No isolated muon back to back with $\mathbb{E}_{T}, \Delta \phi_{\mathbb{E}_{T}, \mu}<165^{\circ}$ 
Table 6.2. Number of data events surviving selection cuts.

\begin{tabular}{|l|r|r|r|}
\hline \multicolumn{3}{|c|}{ Data Events Surviving } \\
\hline \hline Cuts & Run IA & Run IB & Run IC \\
\hline Working Sample (includes cleanup) & 2,398 & 17,055 & 965 \\
\hline Trigger Requirements & 422 & 9,289 & 540 \\
\hline$\not_{T}^{\text {cal }} \geq 20 \mathrm{GeV}$ & 350 & 4,190 & 295 \\
\hline$\not_{T} \geq 20 \mathrm{GeV}$ & 213 & 2,614 & 180 \\
\hline Jet Cuts & 55 & 602 & 43 \\
\hline Good Isolated Muon & 28 & 445 & 25 \\
\hline $\mathrm{H}_{T} \geq 50 \mathrm{GeV}$ & 24 & 316 & 18 \\
\hline Zfitter and back-to-back $\mu / \mathbb{E}_{T}$ & 16 & 256 & 13 \\
\hline Good Tagging Muon & 1 & 8 & 1 \\
\hline
\end{tabular}

- $H_{T} \geq 50 \mathrm{GeV}\left(H_{T}\right.$ is the sum of all jet $E_{T}$ with $E_{T}>15 \mathrm{GeV}$ and $|\eta|<2.0$ )

The definition for $H_{T}$ is not arbitrary but serves to count only hard jets in an $\eta$ region where most of the hard interactions are expected. Each signal is expected to have at least two jets in this region. The $H_{T}$ criterion is effective at rejecting QCD events because the jets in these events tend to be produced at larger $\eta$ than those of the signal.

\subsection{The Heavy Flavor (QCD) Tagrate Function}

The QCD background calculation is discussed in Section 6.5.1. That calculation makes use of a heavy flavor tagrate function. A tagrate function gives the probability for a jet to produce a tagging muon and thus tag the jet. 
The heavy flavor QCD tagrate function for a jet is assumed to be a function of the transverse momentum and $\eta$ of the jet. It also depends on the run number. Furthermore, the jet $p_{T}$ and $\eta$ are assumed to be independent variables. Given this assumption, we can write the tagrate function $R$ in the factorized form $R\left(p_{T}, \eta, r\right)=$ $F\left(p_{T}\right) K(\eta, r)$. The tagrate function for QCD was derived from Monte Carlo b-quark jets and tested on low $\mathbb{E}_{T}$, low jet multiplicity events with at least one jet and a high $p_{T}$ muon [97].

The $p_{T}$ dependence of the QCD tagrate function, $F\left(p_{T}\right)$, is fit to a piecewise function: a quadratic up to $p_{T}=\rho$ and a constant thereafter. The function is said to "saturate" at high $p_{T}$.

$$
F\left(p_{T}\right)= \begin{cases}=A+B p_{T}+C p_{T}^{2} & \text { for } p_{T} \leq \rho \\ =A+B \rho+C \rho^{2} & \text { for } p_{T}>\rho\end{cases}
$$

The value of $\rho$ is $\frac{-B}{2 C}$. Unfortunately, the data are sparse at high $p_{T}$ and therefore do not provide much guidance about the behavior of $F\left(p_{T}\right)$ in that region. Assuming that $F\left(p_{T}\right)$ saturates is the simplest assumption one can make.

We now consider the $\eta$ dependence of the function $K(\eta, r)$. There are three run ranges, $r$, Run IA (1), Run IB prezap (2) and Run IB postzap (3). The $\eta$ dependence is fit to the expressions in Eq. 6.3 for $\mathrm{CF}$ and $\mathrm{EF}$ jets. We note that the form of these expressions has no particular significance; many other functional forms could have been used.

$$
\begin{aligned}
K^{C F}(\eta, r) & =D_{r}^{C F}\left(1+G_{r}^{C F}|\eta|^{2}\right)\left[\operatorname{erf}\left(|\eta| E_{r}^{C F}\right)+F_{r}^{C F}-\operatorname{erf}\left(|\eta| E_{r}^{C F}-F_{r}^{C F}\right)\right], \\
K^{E F}(\eta \prime, r) & =D_{r}^{E F}\left[\operatorname{erf}\left(|\eta \prime| E_{r}^{E F}+F_{r}^{E F}\right)-\operatorname{erf}\left(|\eta \prime| E_{r}^{E F}-F_{r}^{E F}\right)\right], \\
\eta \prime & =|\eta|-G_{r}^{E F} .
\end{aligned}
$$

In Eq. 6.3, the subscript $r$ labels the run range and $\operatorname{erf}(t)=2 / \sqrt{\pi} \int_{0}^{x} \exp \left(-t^{2}\right) d t$. The parameters $D_{r}, G_{r}, E_{r}$, and $F_{r}$ are fit to QCD Monte Carlo data. 
Table 6.3. QCD tagrate function parameters.

\begin{tabular}{|c|c|c|c|c|c|}
\hline \multicolumn{6}{|c|}{$K^{C F}(\eta, r)$ Parameters } \\
\hline \multirow{4}{*}{\multicolumn{2}{|c|}{$F\left(p_{T}\right)$ Parameters }} & Run & $\mathrm{IA}(\mathrm{r}=1)$ & IB-prezap $(r=2)$ & IB-postzap $(\mathrm{r}=3)$ \\
\hline & & $D_{r}^{C F}$ & 0.5 & 0.5 & 0.5 \\
\hline & & $E_{r}^{C F}$ & 11.45 & 2.264 & 4.783 \\
\hline & & $F_{r}^{C F}$ & 12.37 & 2.166 & 4.846 \\
\hline A & -0.14191 & $G_{r}^{C F}$ & -0.4825 & -0.4766 & -0.1982 \\
\hline $\mathrm{B}$ & $0.91180 \mathrm{E}-02$ & & & & \\
\hline \multirow[t]{6}{*}{$\mathrm{C}$} & $-0.67532 \mathrm{E}-04$ & \multicolumn{4}{|c|}{$K^{E F}(\eta, r)$ Parameters } \\
\hline & & Run & $\mathrm{IA}(\mathrm{r}=1)$ & IB-prezap $(r=2)$ & IB-postzap $(\mathrm{r}=3)$ \\
\hline & & $D_{r}^{E F}$ & 0.0 & 0.0 & $0.3489 \mathrm{E}-02$ \\
\hline & & $E_{r}^{E F}$ & 0.0 & 0.0 & 3.919 \\
\hline & & $F_{r}^{E F}$ & 0.0 & 0.0 & 1.539 \\
\hline & & $G_{r}^{E F}$ & 0.0 & 0.0 & 1.434 \\
\hline
\end{tabular}

In order to calculate a tagrate value for an event, the tagrate for each jet is calculated and then the tagrates are summed, as shown in Eq. 6.4. For a jet to contribute to the calculation, it must be untagged and have $p_{T}>15$ and $|\eta|<2.5$.

$$
R_{\text {Event }}=\sum_{j} R\left(p_{T}^{j}, \eta^{j}, r\right) .
$$

Typical values per jet for the QCD heavy flavor tagrate function vary, but are typically $0.012 \pm 0.001$ to $0.018 \pm 0.002$ for the highest $E_{T}$ jet in a typical preselected signal event. The EF contribution to the QCD tagrate is small because the number of tagging muons in the EF is small as is clear from the $\eta$ distributions for the tagging muons, shown in Fig. 6.1. 


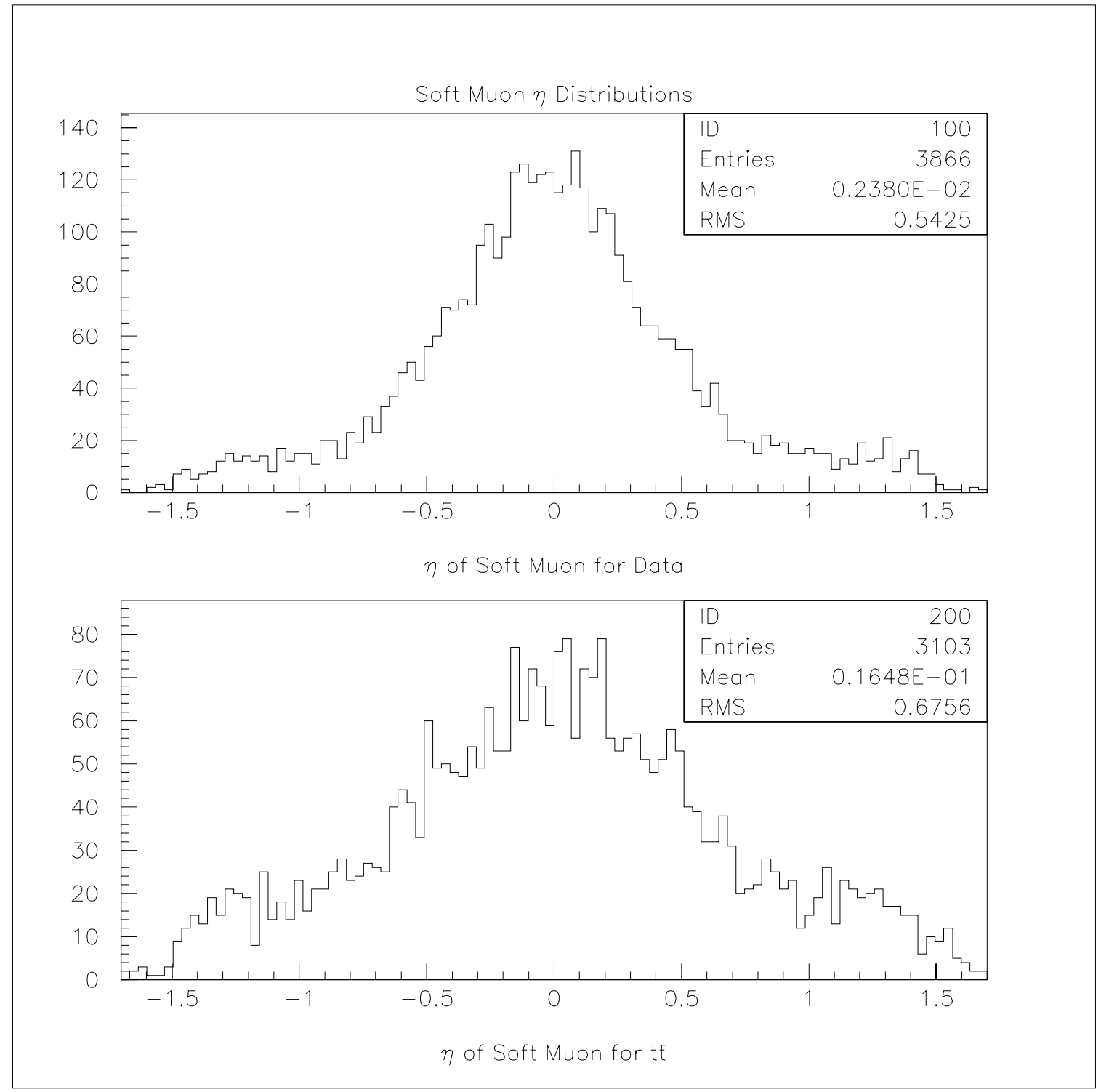

Figure 6.1. $\eta$ distributions of the soft muon for a data background sample and a $t \bar{t}$ sample of events. The $t \bar{t}$ sample was chosen because of its enrichment of b-quark jets. 


\subsection{Backgrounds}

A important part of understanding the data selected, is the understanding of the backgrounds. The background model is especially important when the signal is much smaller than the background, as is the case with single top. A variety of tools are used to model the backgrounds, including background Monte Carlo events and data.

\section{Herwig $t \bar{t}$ Monte Carlo}

The primary backgrounds to the single top muon channel are $t \bar{t}$ and $W+$ jets. For the $t \bar{t}$ process, a sample of events has been obtained from the top D $\varnothing$ group. This sample used Herwig 5.7 [80] for event generation and fragmentation. The scale used was the Herwig default scale. CTEQ3M [9] was used for the parton distribution function with a top mass of $170 \mathrm{GeV}$. There were 81,141 events in this sample (which included all leptonic decays of the $\mathrm{W}$ ). The $t \bar{t}$ Monte Carlo is described in several top thesis [81-83].

\section{Vecbos $W+$ jets Monte Carlo}

The $W+$ jets backgrounds have been modeled using VECBOS 3.0 [84], fragmented with Herwig 5.7 [80]. In the base sample, there were 116,386 events (which included any leptonic decay of the $\mathrm{W})$. The $W+$ jets background has been studied in detail by physics groups at D $\varnothing[81]$.

\subsubsection{QCD Multi-Jet Background to the $\mu$-tag Analysis}

Occasionally, a multi-jet system can fake an isolated $\mu+$ jets system if the jet, associated with the muon, is lost by being below reconstruction threshold or if the muon falls outside of the jet cone. The rate at which this happens is called the fake rate. The QCD background arising from lost jets is called the instrumental QCD background. The other source of QCD background is the result of a physical process, 
rather than a deficiency in reconstruction. The muon from a tagged jet can appear outside the jet cone and, therefore, be incorrectly identified as an isolated muon, that is, a muon not associated with a jet. This background is called the physical QCD background. The physical and instrumental backgrounds have the same final state and it is impossible to determine if the putative isolation arises from a physical process or an instrumental one.

To calculate the QCD background, we determine the fake rates for instrumental and physical isolation in a region where one expects little or no contamination from other background processes; in our case, the region is defined by $\not_{T}<20 \mathrm{GeV}$. A key assumption is that the fake rates are independent of the $\mathscr{E}_{T}$. This assumption has been tested by varying the $\mathscr{E}_{T}$ cuts, see Fig. 6.2

Table 6.4. Fake rates as a function of $\mathbb{H}_{T}$ for $\mathrm{CF}$ muons only. The result for $\mathrm{EF}$ muons are similar. The cuts used here do not reject $\mathrm{Z}+$ jets events or mismeasured $\mathbb{E}_{T}$ events.

\begin{tabular}{|c|c|c|c|}
\hline \multirow{2}{*}{$\begin{array}{c}\text { Jet } \\
\text { Multiplicity }\end{array}$} & \multicolumn{3}{|c|}{ Fake Rate } \\
\cline { 2 - 4 } & $\mathscr{E}_{T}<15 \mathrm{GeV}$ & $\mathscr{E}_{T}<20 \mathrm{GeV}$ & $\mathscr{E}_{T}<25 \mathrm{GeV}$ \\
\hline 1 & $.131 \pm 0.006$ & $.134 \pm 0.005$ & $.139 \pm 0.005$ \\
\hline 2 & $.067 \pm 0.006$ & $.085 \pm 0.006$ & $.096 \pm 0.006$ \\
\hline 3 & $.049 \pm 0.011$ & $.066 \pm 0.011$ & $.073 \pm 0.011$ \\
\hline 4 & $.058 \pm 0.028$ & $.052 \pm 0.022$ & $.052 \pm 0.021$ \\
\hline 5 & $.143 \pm 0.093$ & $.200 \pm 0.089$ & $.143 \pm 0.066$ \\
\hline
\end{tabular}

The instrumental QCD background for an N-jet system is calculated by multiplying the number of $\mathrm{N}+1$-jet non-isolated muon events satisfying all analysis cuts in Section 6.3, except the isolation requirement for the high $p_{T}$ muon, by the probability that an isolated $\mu+$ jet system appears, and by the heavy flavor tagrate. 


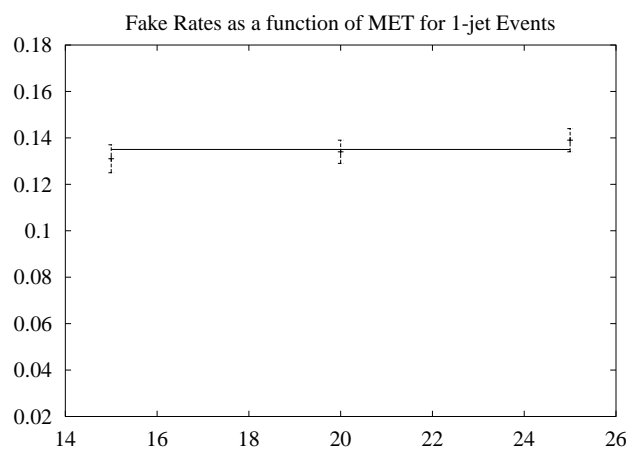

(a) One Jet

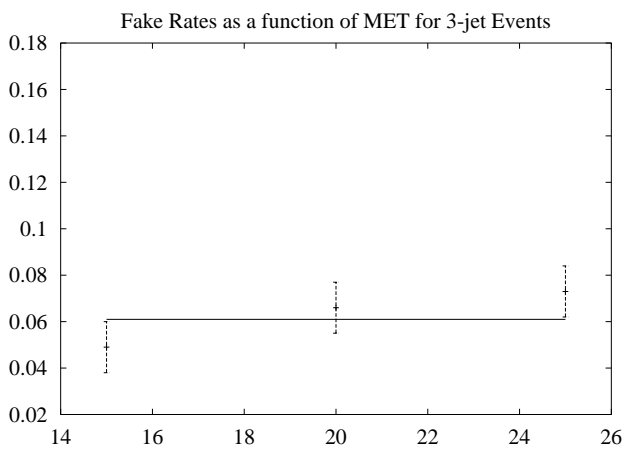

(c) Three Jets

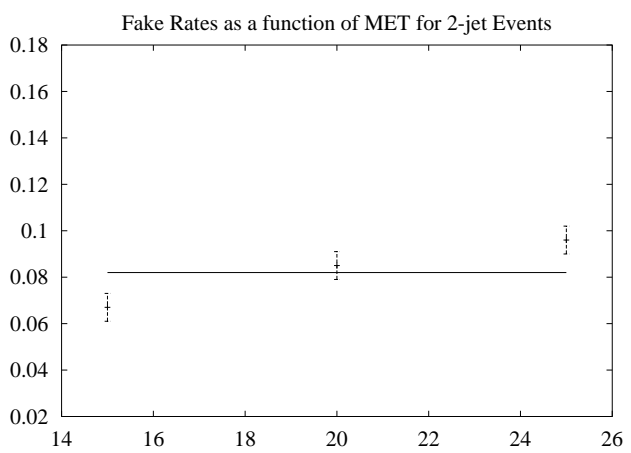

(b) Two Jets

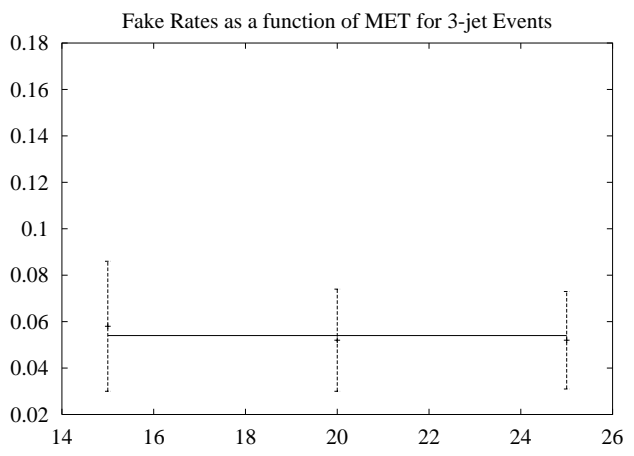

(d) Four Jets

Figure 6.2. Isolated muon fake rates as a function of Missing Transverse Energy. A non-isolated muon is a muon which satisfies all of the isolated muon requirements except isolation, $\Delta R<0.5$. The heavy flavor tagrate functions models the rate at which heavy flavor quarks produce a tagging muon. The heavy flavor tagrate is discussed in Section 6.4.

The QCD background was calculated from a very loose background sample of events. These events required no isolated muons and one or more CAFIX corrected jets. The QCD sample is expected to be contaminated by the $W+$ jets backgrounds and possibly other backgrounds that produce a fake isolated muon: 


$$
\begin{aligned}
F_{R} & =\frac{N_{n j}^{I}}{N_{n j+1}^{N I}} \\
N_{\text {tag }} & =F_{R} \sum_{\text {events }} \sum_{j e t s} T_{R}^{N I p}
\end{aligned}
$$

where

- $F_{R}$ is the QCD fake rate.

- $N_{n j}^{I}$ is the number of QCD isolated muon events, with $\mathbb{E}_{T}<20 \mathrm{GeV}$ and nj jets.

- $N_{n j+1}^{N I}$ is the number of QCD non-isolated muon events, with $\mathbb{E}_{T}<20 \mathrm{GeV}$ and $n j+1$ jets.

- $T_{R}^{N I p}$ is the number of QCD non-isolated muon events with $\not E_{T}>20 \mathrm{GeV}$ weighted by the heavy flavor tagrate.

- $N_{\text {tag }}$ is the calculated number of QCD isolated muon multi-jet tagged events.

The fake rate, $F_{R}$, is determined from events with $\mathbb{E}_{T}<20 \mathrm{GeV}$ and passing a MU_JET_XXX trigger. To enter the background calculation, a QCD event must pass the same analysis cuts as the selected data and have a non-isolated muon.

The QCD multi-jet background and the $W+$ jets background calculations are, to first order, orthogonal calculations. At the root of the issue is the nomenclature. We do not dispute the fact that the QCD multi-jet calculation is not a pure sample with only the instrumental and the physical QCD processes. On the contrary, we note the QCD multi-jet background necessarily contains $W+$ jets contributions and vice versa. We will not declare that the samples themselves are orthogonal however, we will show that the calculations themselves are orthogonal.

The $W+$ jets events are calculated from events which pass all selection cuts (except the tagging muon requirement), see Section 6.3. These events must have $E_{T}>20 \mathrm{GeV}$ and an isolated muon. The QCD multi-jet events that contribute 
have only non-isolated muons. The QCD fake rate is calculated from a sample of events which fail the $\mathbb{E}_{T}$ cut. Thus, the two samples are mutually exclusive. There is no contribution from the $W+$ jets background calculation to the QCD multi-jet background calculation and no contribution from the QCD multi-jet background contribution to the $W+$ jets calculation.

As an aside, we can quantify how much $W+$ jets events are in the QCD multi-jet sample and vice versa from Appendix D and $W+$ jets Monte Carlo. For the QCD fake rate calculation, the primary component, from $W+$ jets events which fail the $\mathbb{E}_{T}$ cut, is found to be $18 \%$. For the QCD multi-jet contribution with a non-isolated muon and $\mathbb{E}_{T}>20 \mathrm{GeV}$ the $W+$ jets contribution is less than $5 \%$. The $W+$ jets sample contains approximately 17\% QCD multi-jet events. Although the QCD backgrounds necessarily contain some $W+$ jets events, the calculation proceeds with the caveat that the $W+$ jets to the QCD contribution is small.

The QCD background results for both the CF and EF are shown in Table 6.7. Table 6.7 shows the number of isolated N-jet events, the number of non-isolated $\mathrm{N}+1$-jet events failing the cuts, the fake rate determined from these samples and the amount of QCD multi-jet tagged background.

In Tables 6.5 and 6.6, the calculation is broken into two parts: one for the CF and one for the EF. The number of non-isolated and isolated events failing the $\mathbb{E}_{T}$ cuts is shown along with the fake rate, expected number of untagged QCD events and the number of non-isolated events passing all cuts. Table 6.7 is derived from these tables.

The trigger efficiency increases with the number of muons in the sample. So, we expect to have slightly better efficiency for the QCD background when it is tagged. The increase in trigger efficiency was determined to be $16 \pm 1.6 \%$. The increase was 
determined from a $W+$ jets Monte Carlo sample with the same topology as the QCD multi-jet tagged background.

The energy scale systematic error dominates the errors at $24 \%$. The energy scale systematic is determined by fluctuating the energies of the jets by: $\pm 1 \mathrm{GeV} \pm 4.5 \%$. There is an additional systematic error from the tagrate of about $7 \%$ determined by testing the tagrate on different samples of data. The total QCD multi-jet background is calculated to be $2.18 \pm 0.54$. The error includes both systematic and statistical errors. 

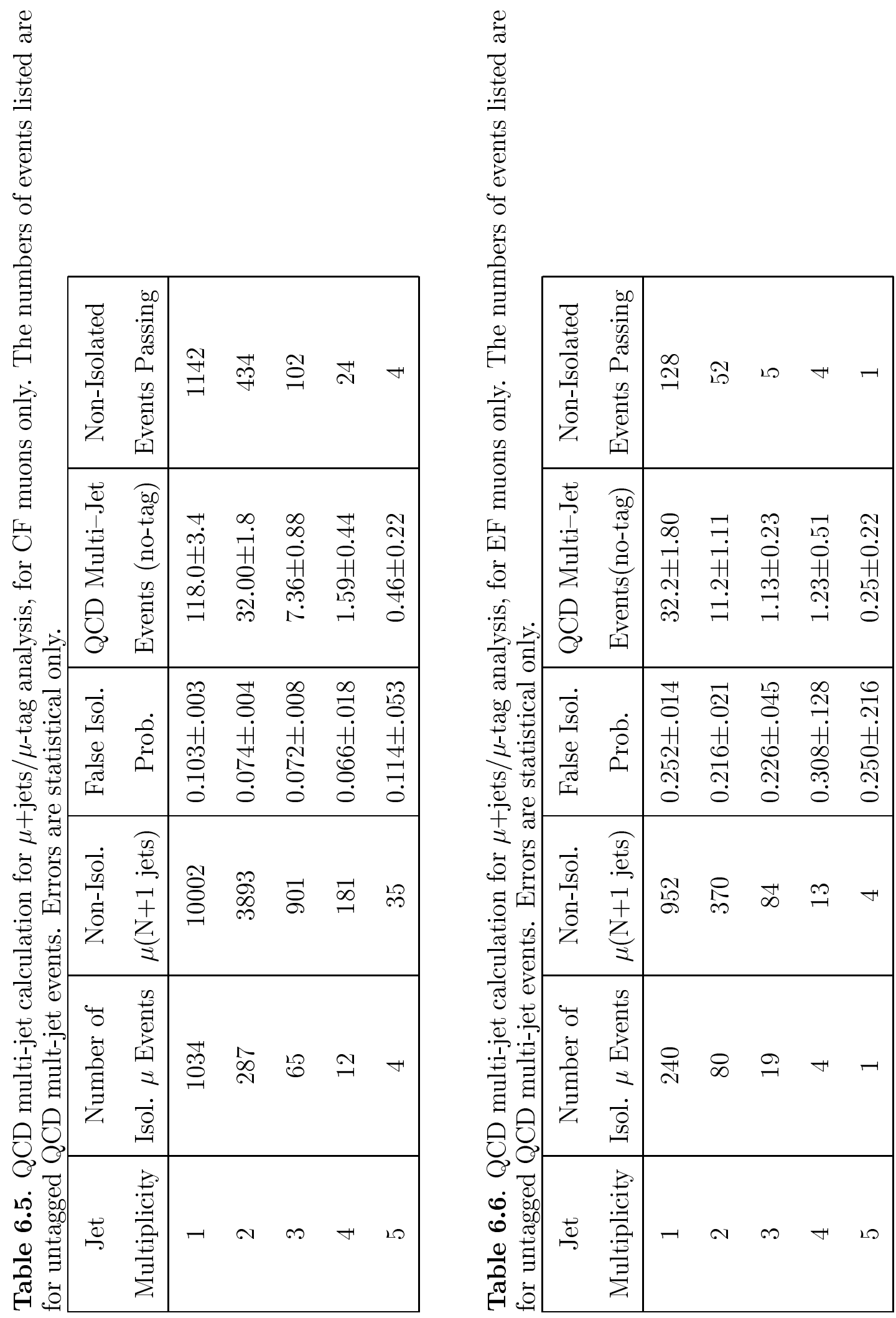


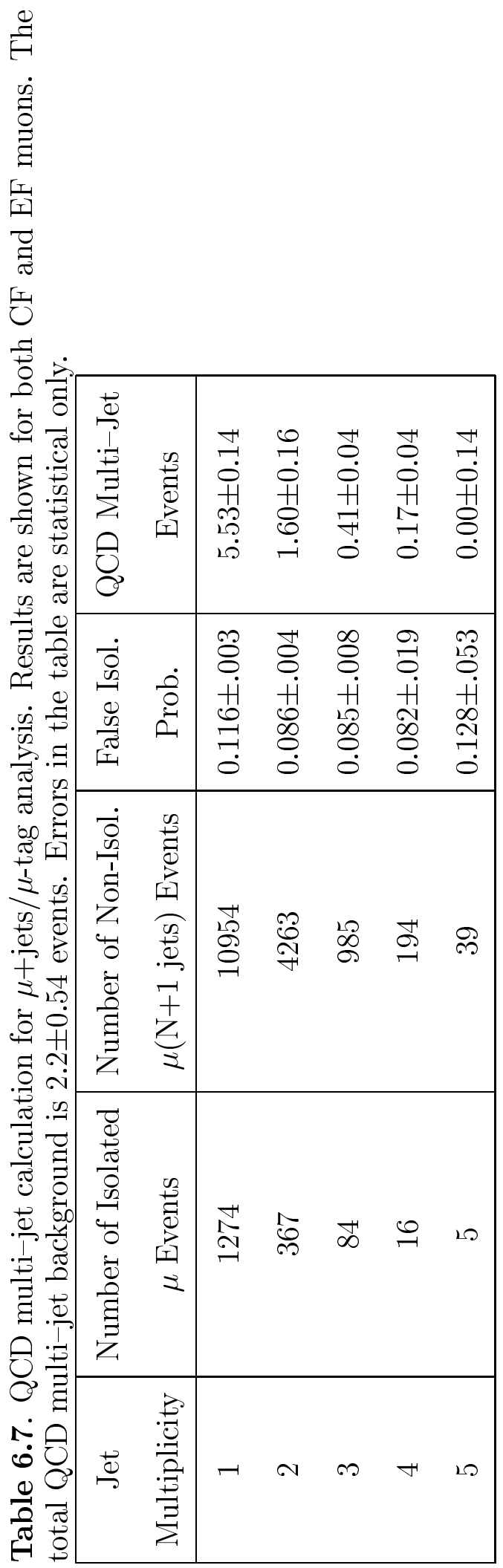




\subsubsection{W+jet background}

The $\mathrm{W}+$ jets background is calculated using one of several flavor-blind tagrate functions. The tagrate functions were extracted from QCD multi-jet data where the jet multiplicity is, in general, much higher than for single top events. However, the tagrate function is determined on a jet by jet basis and the overall jet multiplicity of the event does not contribute to the tagrate function.

A tagrate function was developed by the top group [85]. In order to calculate the background from the data, we require untagged events which pass the muon+jets untagged requirements, then apply the tagrate function to each jet in the event.

An important test of the tagrate function is to show that untagged data correctly models the tagged data, when weighted by the tagrate function. We use the Tagrate7, a flavor-blind tagrate function developed by the top group, and compare some distributions of tagged data with tagrate weighted untagged data. The results are shown in Figure 6.6. The Kolmogorov test was used to compare the likeness of two distributions. The agreement, determined by the Kolmogorov test, is good, though the statistics are somewhat limited for this test.

For Run IB, the tagrate function is used to predict a total $W+$ jets background of $2.27 \pm 0.20$ (stat) \pm 0.50 (sys) events. There is an increased trigger efficiency when a second muon is found. From a $W+$ jets Monte Carlo sample, this increase was found to be $16 \% \pm 1.6 \%$. The systematic error for the tagrate function is $9 \%$. The total $W+$ jets background is $2.73 \pm 0.53$.

\subsection{3 tt Backgrounds}

The primary contribution to the single top background is the $t \bar{t}$ background because it has a signature very similar to single top events. However, the $t \bar{t}$ events have more and higher $p_{T}$ jets. In order to keep as much acceptance for single top as possible, we keep events with up to five jets and events with high $p_{T}$ jets. 


\section{QCD Multi-Jet Event with an Isolated Muon}

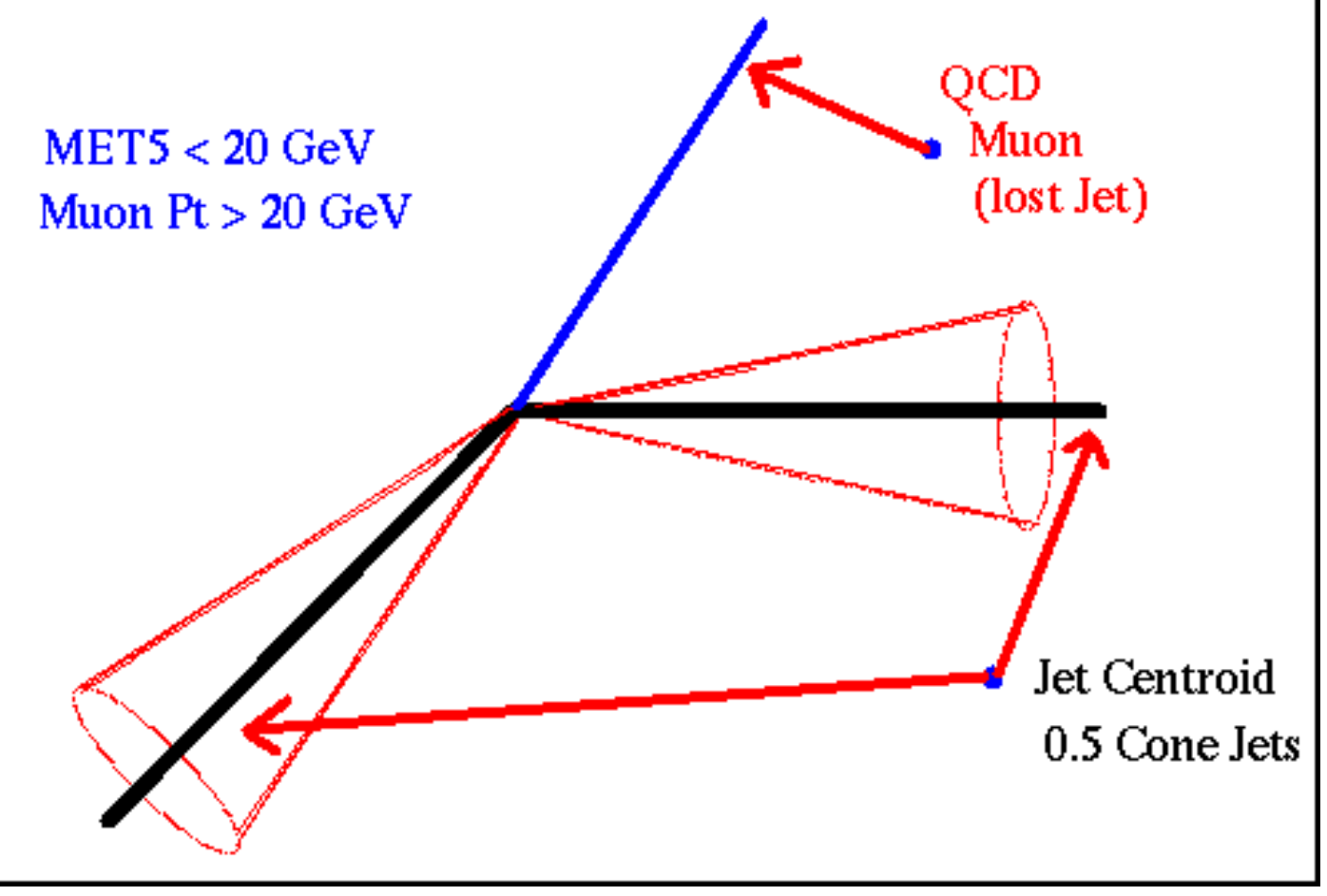

Figure 6.3. An isolated QCD event in which a jet is lost. The assumption here is that if we had a perfect detector, there would be no instrumental QCD events where a muon is not inside the cone of a jet. However, the physical process in which muons escape the cone of a jet would contribute to a QCD multi-jet background. In the low $E_{T}$ region, there is little $W$ contamination and the events are assumed to be mostly QCD.

We calculate the $t \bar{t}$ background using the DØ measured $t \bar{t}$ cross section, and acceptances based on $t \bar{t}$ Monte Carlo events. To calculate the number of expected events we use $\sigma=5.5 \pm 1.8 \mathrm{pb}$ [86] and a trigger efficiency for top events with a tagged and an isolated muon of $\epsilon_{t r i g}^{t o p(2 \mu)}=73.7 \% \pm 7.3 \%$. There are at least two heavy flavor jets in $t \bar{t}$ events. The branching fraction for $b \rightarrow \mu$ is $10.8 \pm 0.05 \%$ per $b$-jet [16]. For a $t \bar{t}$ event, the probability times geometric acceptance for one of the two b-jets to produce a tagging muon is $B R^{a c c}(b \rightarrow \mu)=23.17 \%$. The $B R^{a c c}(b \rightarrow \mu)$ is the 


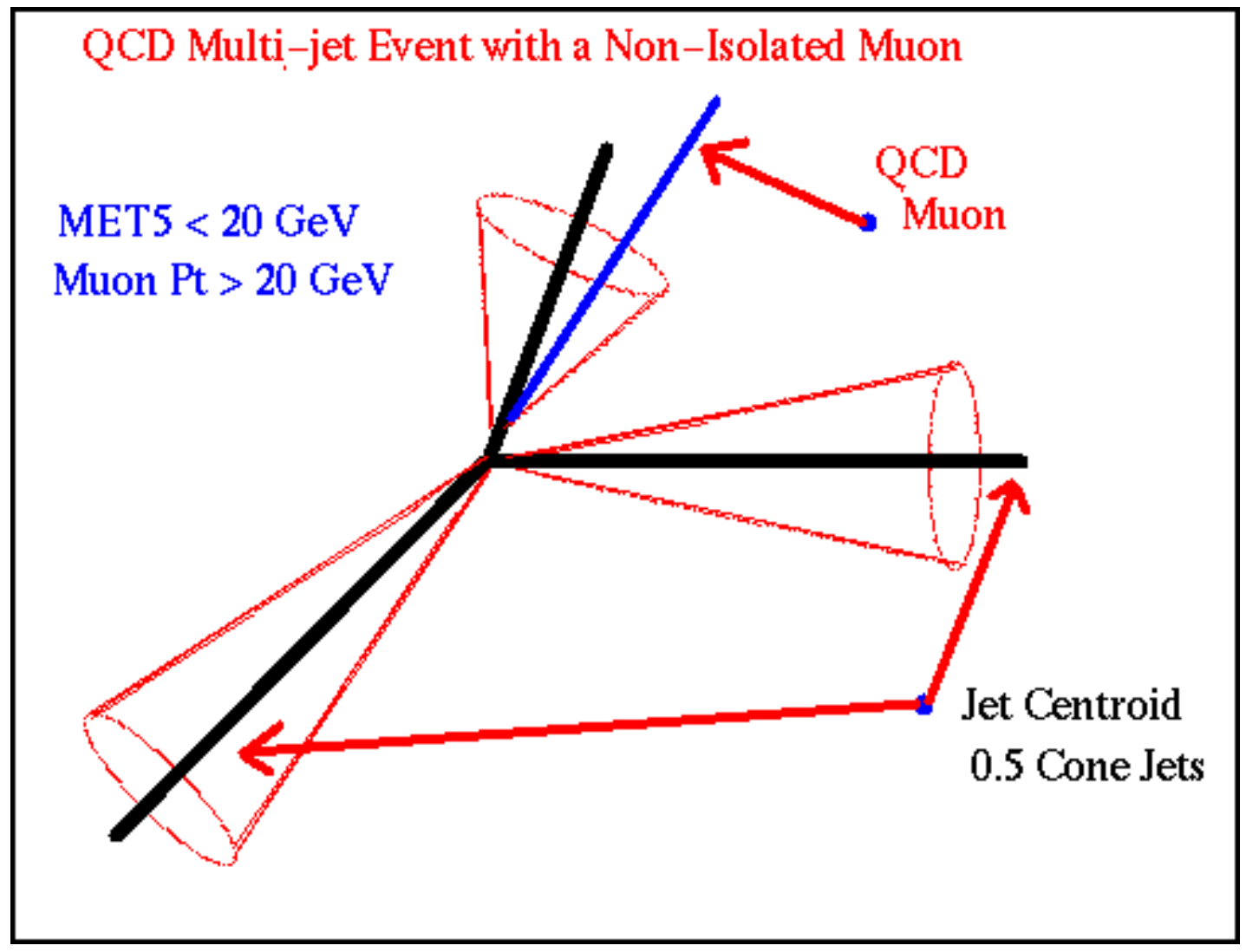

Figure 6.4. A non-isolated QCD event. The muon meets all the requirements of an isolated one, but fails the isolation requirement $\Delta R>0.5$.

branching fraction for $b \rightarrow \mu$ times the geometric acceptance which is determined from Monte Carlo events. The branching fraction for $\mathrm{b} \rightarrow \mu$ used in the Monte Carlo program is in agreement with values calculated from the Particle Data Group. There is an additional contribution to $B R^{a c c}(b \rightarrow \mu)$ in $t \bar{t}$ production from the heavy flavor hadronic decay of the $W$. After the $t \bar{t}$ events pass the selection requirements, we find the kinematic acceptance for $t \bar{t}$ events to be $A c c_{g e o}=0.0974 \pm 0.0098$ from Monte Carlo studies.

The $A c c_{g e o}$ was determined from a sample of events where the $W$ from top was allowed to decay into any leptonic channel. Thus, a factor $B R(t \bar{t} \rightarrow \mu+$ 


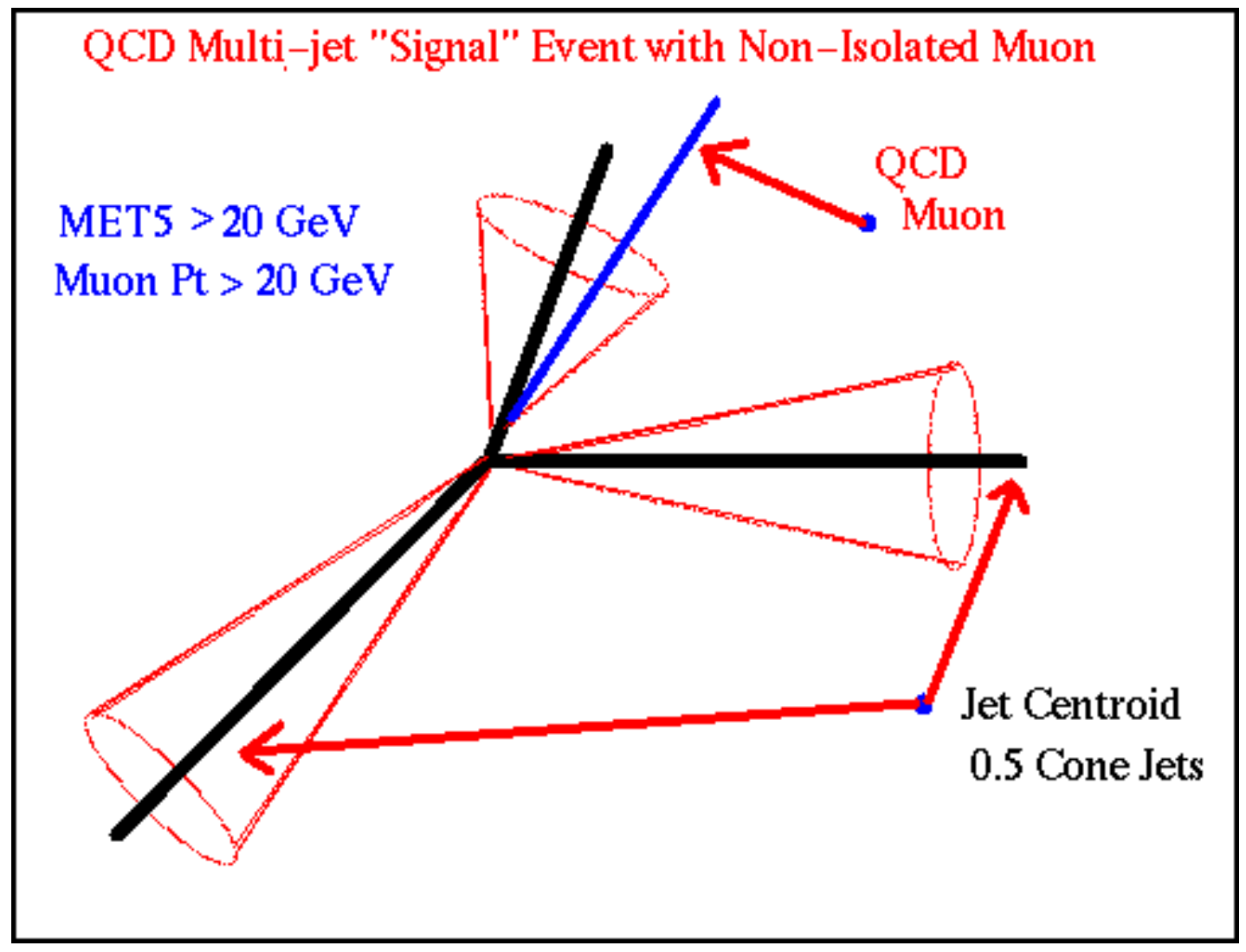

Figure 6.5. A non-isolated QCD event passing all analysis cuts, except muon isolation. The number of fake events is determined from the number of events of this type multiplied by the fake rate (and convolved with the heavy flavor tagrate for QCD).

jets $/ \mu)=0.4557$ was used to calculated the expected number of events from $t \bar{t}$ production.

Using the above numbers, we can calculate, using

$$
\begin{aligned}
\epsilon_{\text {top }} & =A c c_{\text {geo }} \times B R^{a c c}(b \rightarrow \mu) \times \epsilon_{\text {trig }}^{\text {top }(2 \mu)}, \\
N_{\text {top }} & =\sigma \times B R(t \bar{t} \rightarrow \mu+\text { jets } / \mu) \times L \times \epsilon_{t o p},
\end{aligned}
$$

the $t \bar{t}$ background in $L=85.6( \pm 5.4 \%) \mathrm{pb}^{-1}$ of data. We find the $t \bar{t}$ contribution to the single top muon + jets tagged analysis to be $3.17 \pm 1.09$ events. Using the eye-scan efficiencies, the muon identification efficiency for top, $\epsilon_{\mu}^{I D}$, is $88.5 \%$. 

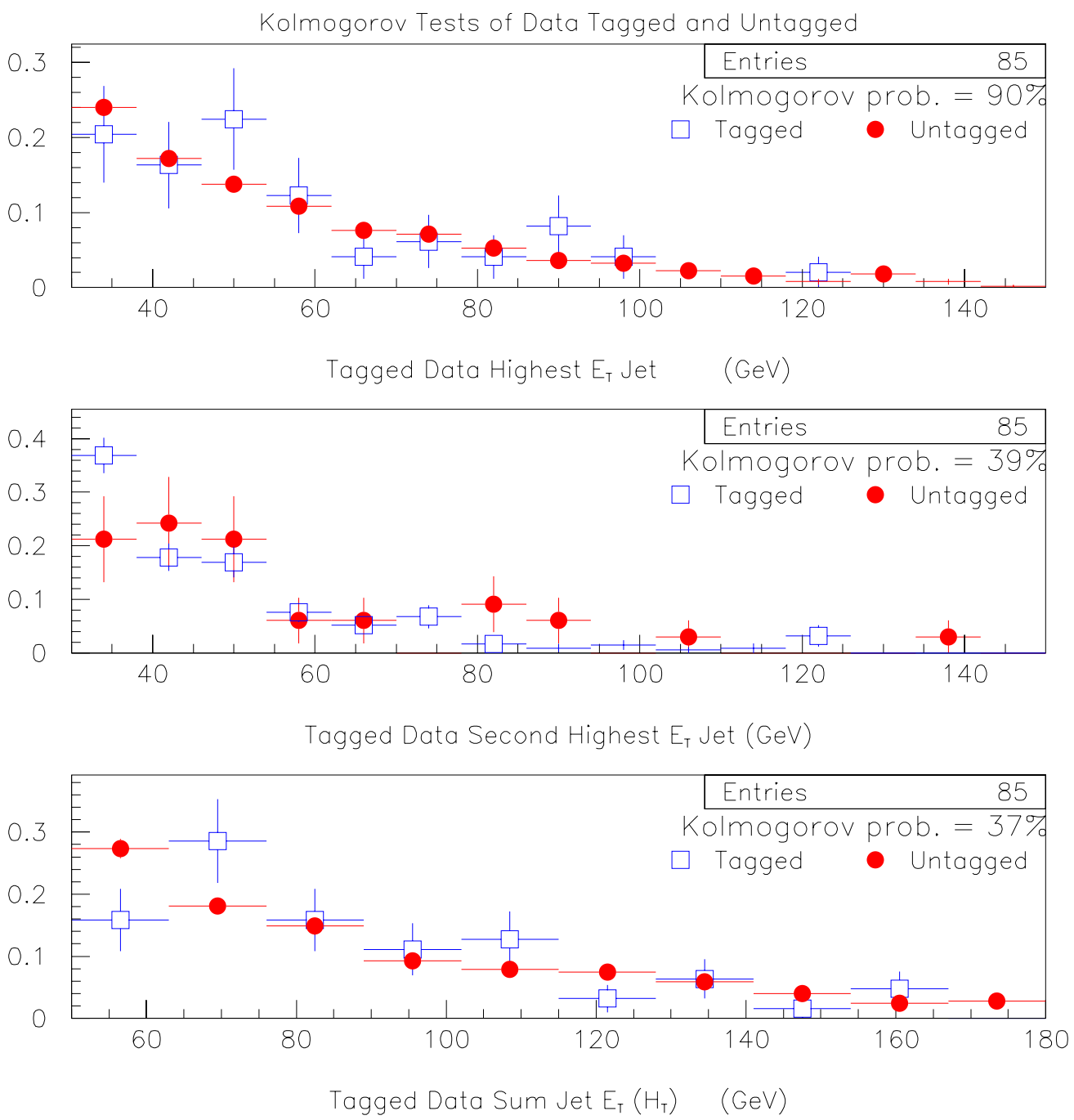

Figure 6.6. Tagged data and untagged data using tagrate7. The tagged data are shown as open squares and the untagged are shown as filled circles. The event selection has been relaxed for a factor of three additional statistics. The cuts applied were 2 or more jets and an isolated (untagged) or tagging (tagged) muon. 


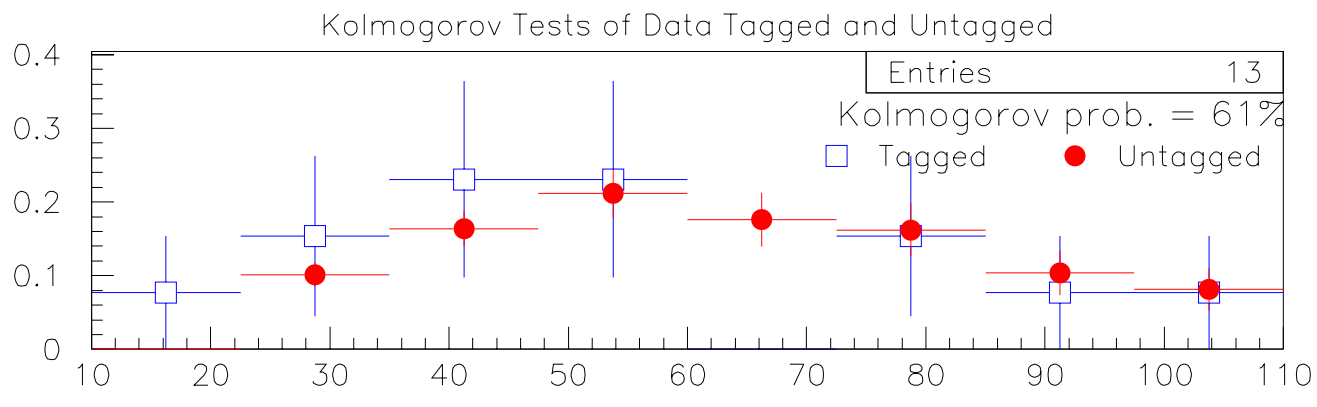

Tagged Data Highest $E_{T}$ Jet $(\mathrm{GeV})$

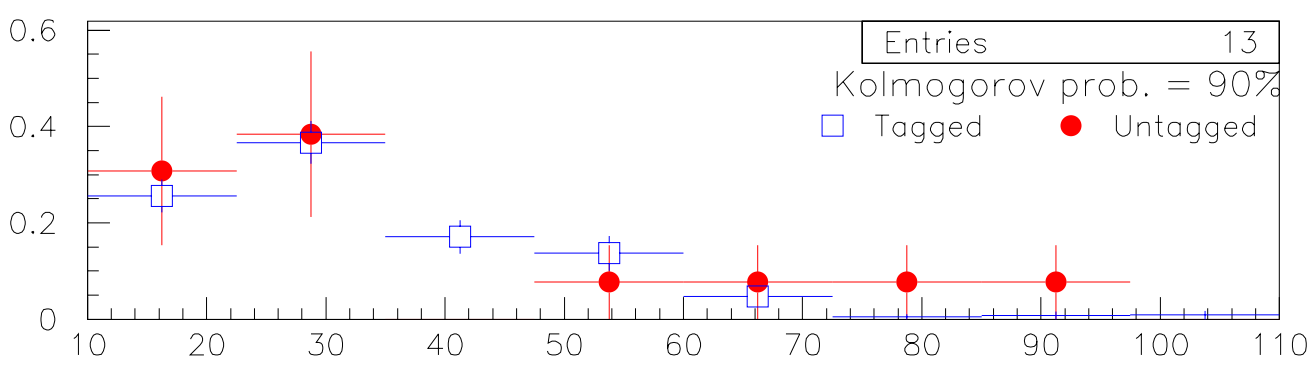

Tagged Data Second Highest $E_{T}$ Jet (GeV)

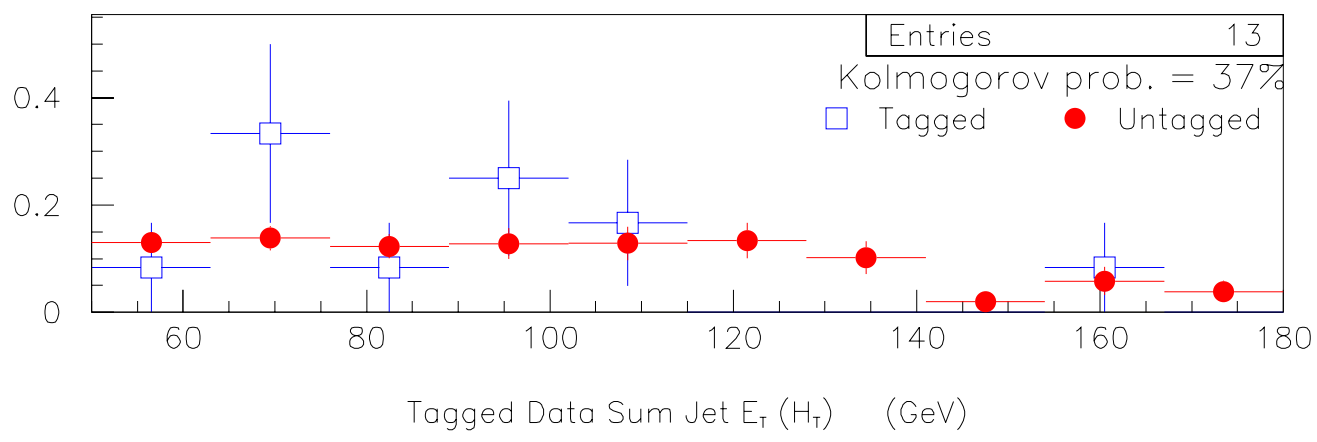

Figure 6.7. Tagged data and untagged data using tagrate7. The tagged data are shown as open squares and the untagged are shown as filled circles. The events shown passed a tight set of cuts, which did not require a tight isolated muon. 

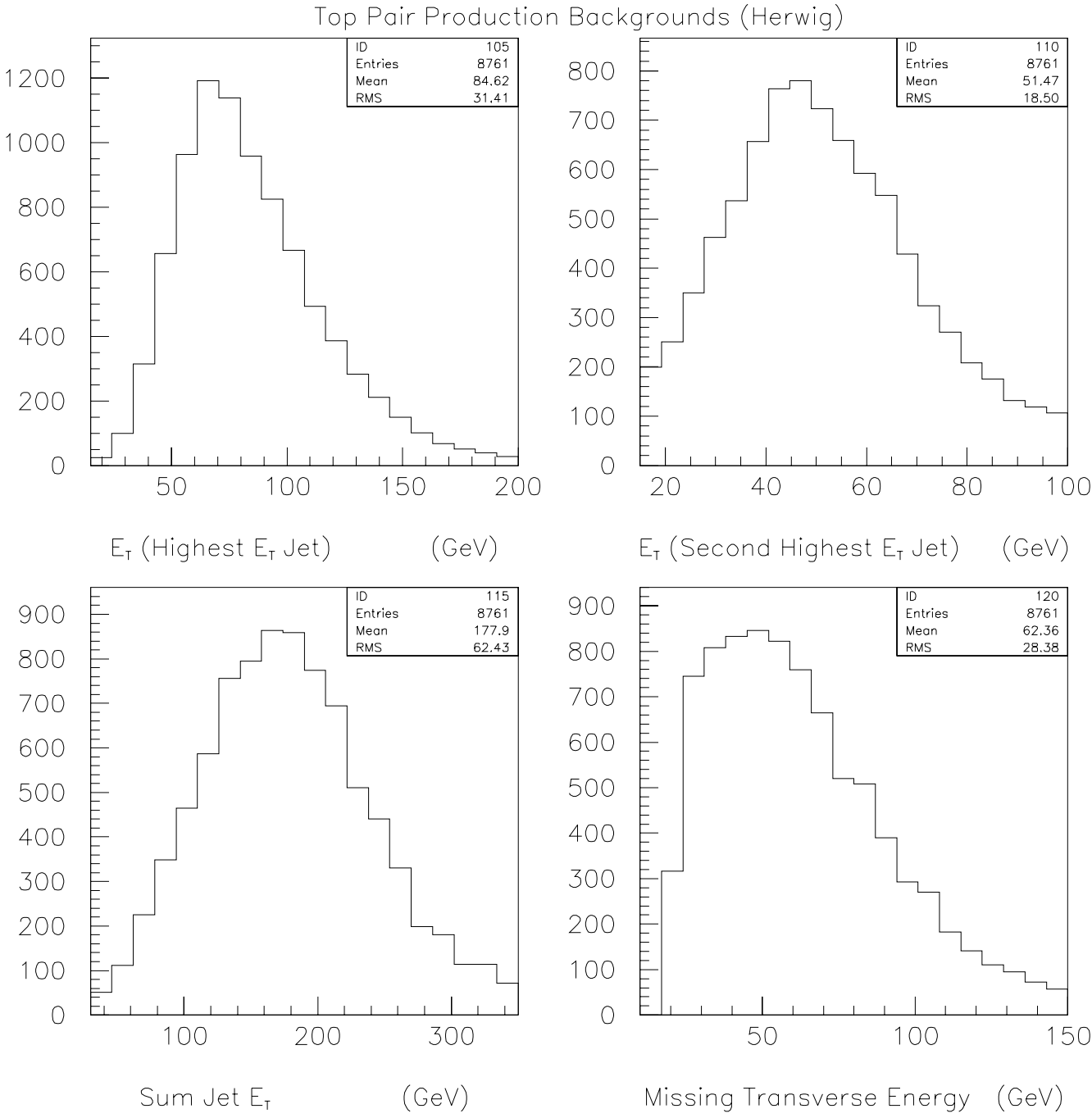

Figure 6.8. The $t \bar{t}$ background kinematic distributions for several variables. The events are subject to selection cuts (see Section 6.3). 

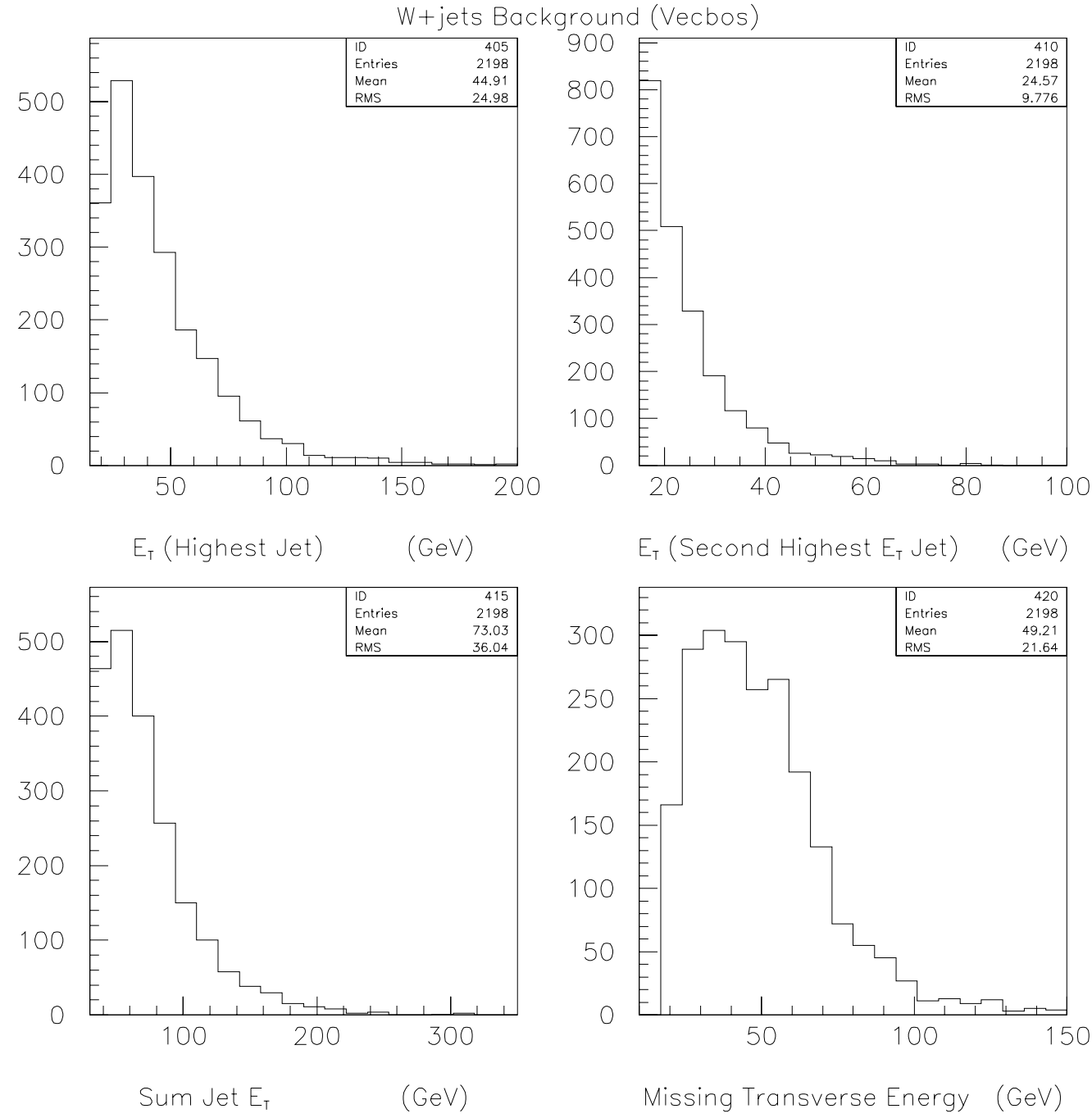

Figure 6.9. The $\mathrm{W}+$ jets background kinematic distributions for several variables. The events are subject to loose selection cuts, those in Section 6.3, without the tagging muon requirement. The model is Vecbos+Isajet. 

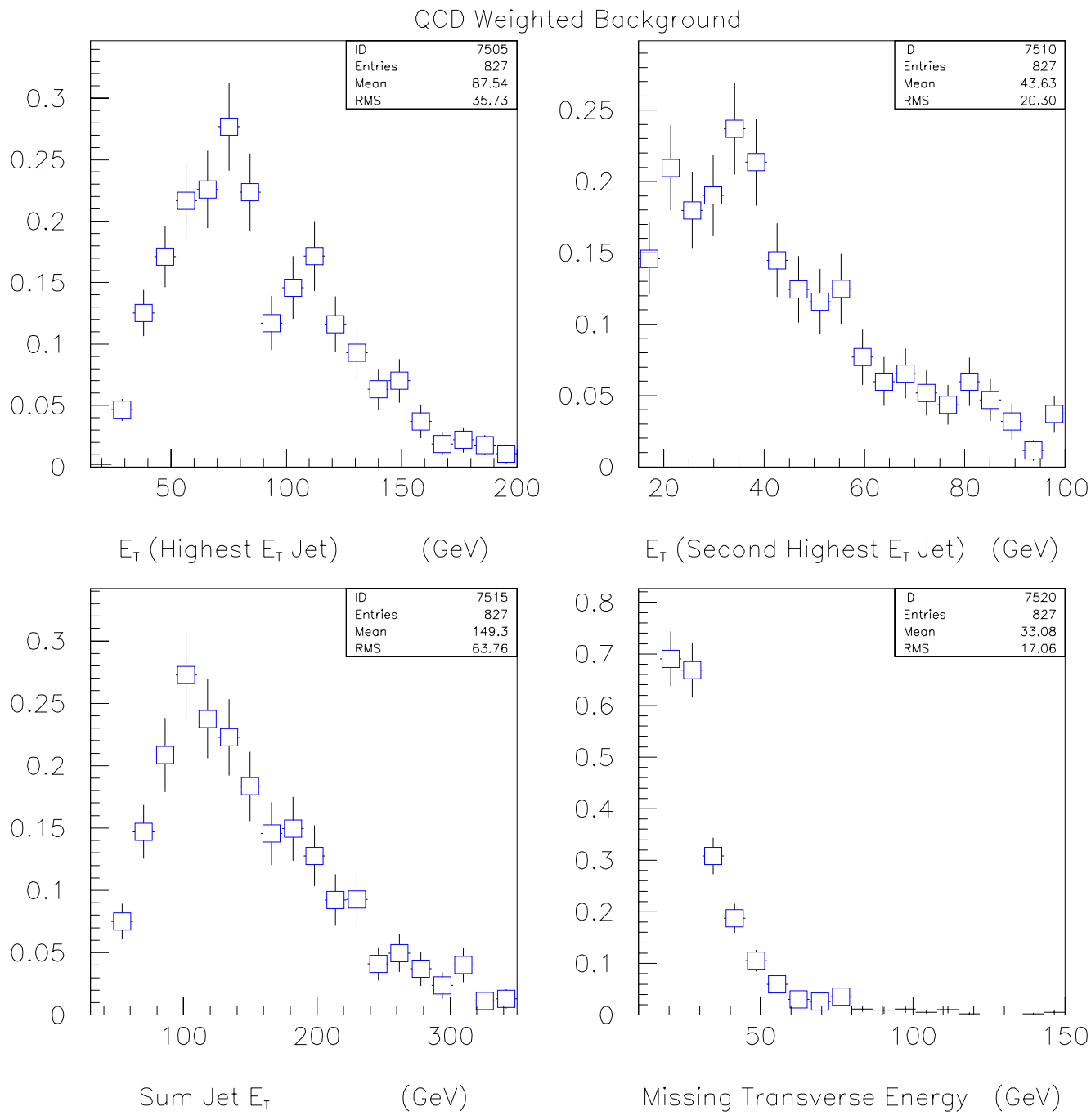

Figure 6.10. The QCD fake isolated background for the $\mu+$ jets/tagged analysis. The events are weighted by the heavy flavor tagrate function. 

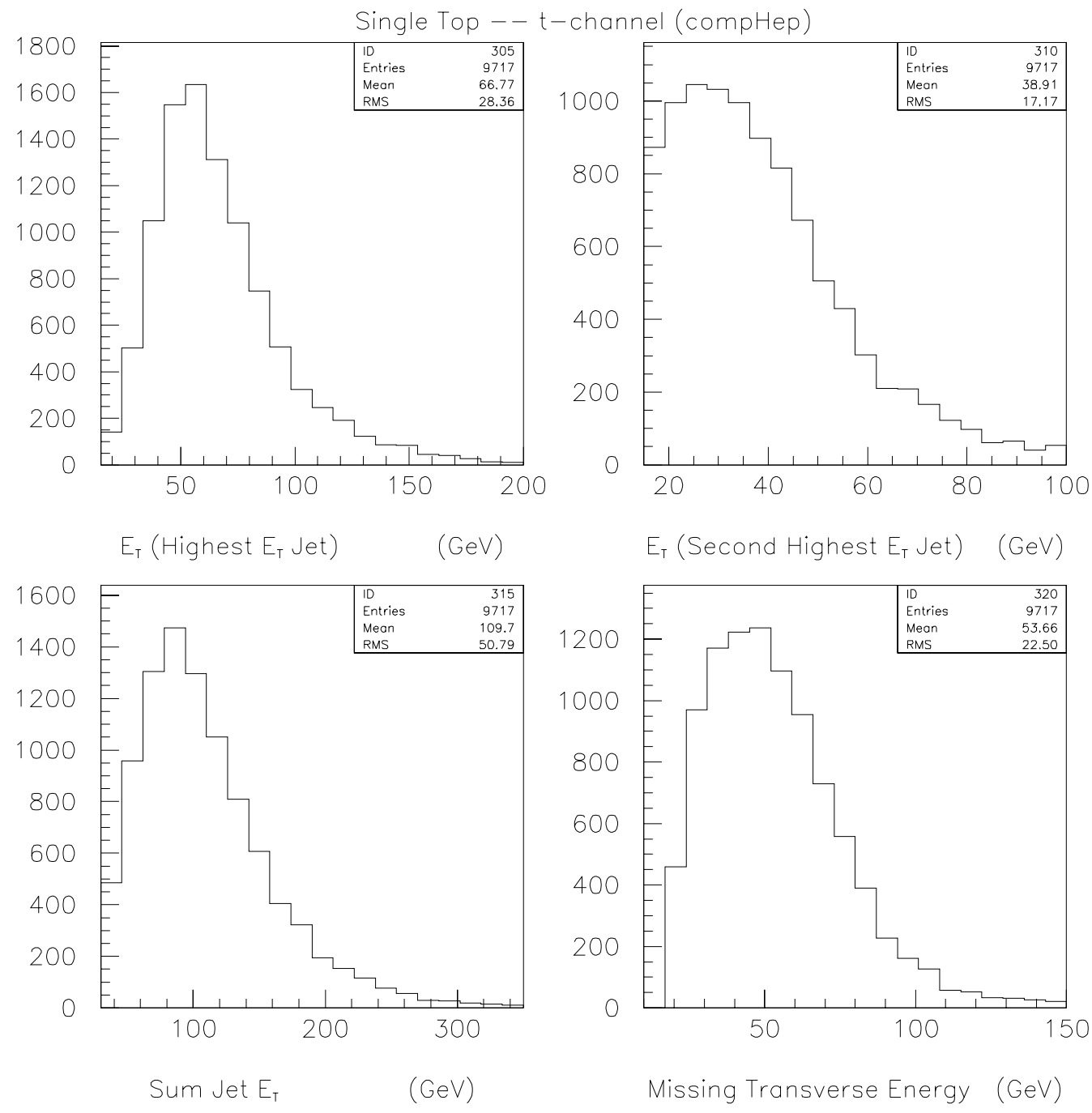

Figure 6.11. The single top t-channel kinematic distributions for several variables. The events are subject to selection cuts (see Section 6.3). The model is CompHEP+PYTHIA. 

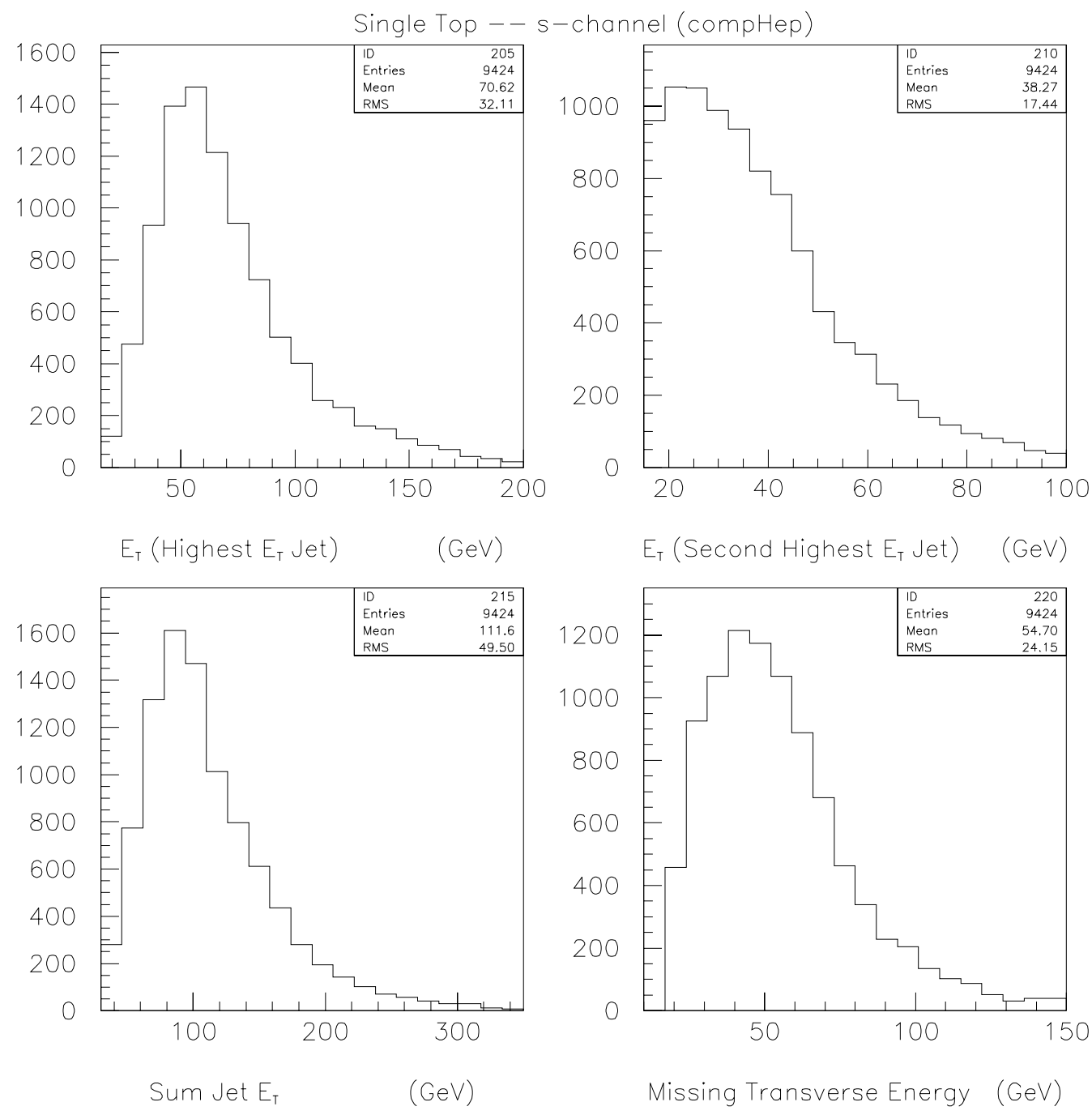

Figure 6.12. The single top s-channel kinematic distributions for several variables. The events are subject to selection cuts (see Section 6.3). The model is CompHEP+PYTHIA. 
Table 6.8. Summary of data events and kinematic quantities. nj ${ }^{\text {Good }}$ is the number of jets with $E_{T}>15$. and $|\eta|<2.5$. This data sample includes all of Run I. An asterisk indicates that the event is a $t \bar{t}$ candidate from previous analyses [74].

\begin{tabular}{|c|c|c|c|c|c|c|c|}
\hline Run/Event & $\mathrm{nj}^{\text {Good }}$ & $\mathrm{Pt}^{j e t 1}$ & $\mathrm{Pt}^{j e t 2}$ & Met5 & $\mathrm{Ht}$ & $\mathrm{Pt}_{\mu}^{i s o l}$ & $\mathrm{Pt}_{\mu}^{\text {soft }}$ \\
\hline $58203 / 4980^{*}$ & 4 & 112.6 & 46.9 & 39.9 & 209.6 & 86.9 & 10.1 \\
\hline 84695/29699* & 3 & 102.4 & 80.6 & 25.8 & 216.5 & 58.6 & 36.2 \\
\hline $88018 / 29231$ & 3 & 45.9 & 31.1 & 29.4 & 95.4 & 28.5 & 5.8 \\
\hline $88044 / 15782$ & 2 & 37.7 & 28.8 & 38.2 & 66.5 & 76.4 & 9.4 \\
\hline $90433 / 25794$ & 2 & 61.4 & 47.6 & 65.7 & 109.0 & 22.6 & 45.5 \\
\hline $90864 / 27059$ & 3 & 30.0 & 33.5 & 30.0 & 63.6 & 51.2 & 7.9 \\
\hline $91206 / 7154$ & 2 & 52.9 & 23.4 & 68.6 & 76.4 & 94.7 & 4.1 \\
\hline $91959 / 6095$ & 2 & 77.1 & 17.7 & 58.4 & 94.8 & 72.4 & 8.3 \\
\hline $92701 / 1130^{*}$ & 2 & 26.4 & 26.4 & 39.1 & 52.9 & 28.4 & 15.0 \\
\hline $96459 / 74957$ & 3 & 83.7 & 26.4 & 26.6 & 116.1 & 49.2 & 10.0 \\
\hline
\end{tabular}

\subsubsection{Total Background to the single top $\mu+$ jets $/ \mu$ channels}

We find a total number of background events for single top $\mu+$ jets $/ \mu$ analysis of 8.1 \pm 1.3 . The total number of events observed in a Run IB sample containing 85.6 $\mathrm{pb}^{-1}$ is 8 . A summary of the kinematic quantities of candidate events is shown in Table 6.8. The addition of Run IA and Run IC contribute an additional $18 \%$ of integrated luminosity. We simply scale the background by this increase in luminosity. The assumption here is that the Run IB data set is representative of the full Run I data set. For the integrated luminosity of $103.7 \mathrm{pb}^{-1}$, we calculate a background of $9.5 \pm 1.52$. During Run IA, punchthrough from the Main Ring was a problem, so all muons with $90^{\circ}<\phi<110^{\circ}$ were rejected. This results in a $5.6 \%$ loss in muon efficiency per muon. During Run IC, a degradation in the muon system led to a decrease in muon efficiencies. A $25 \%$ efficiency loss occurred during Run IC. 


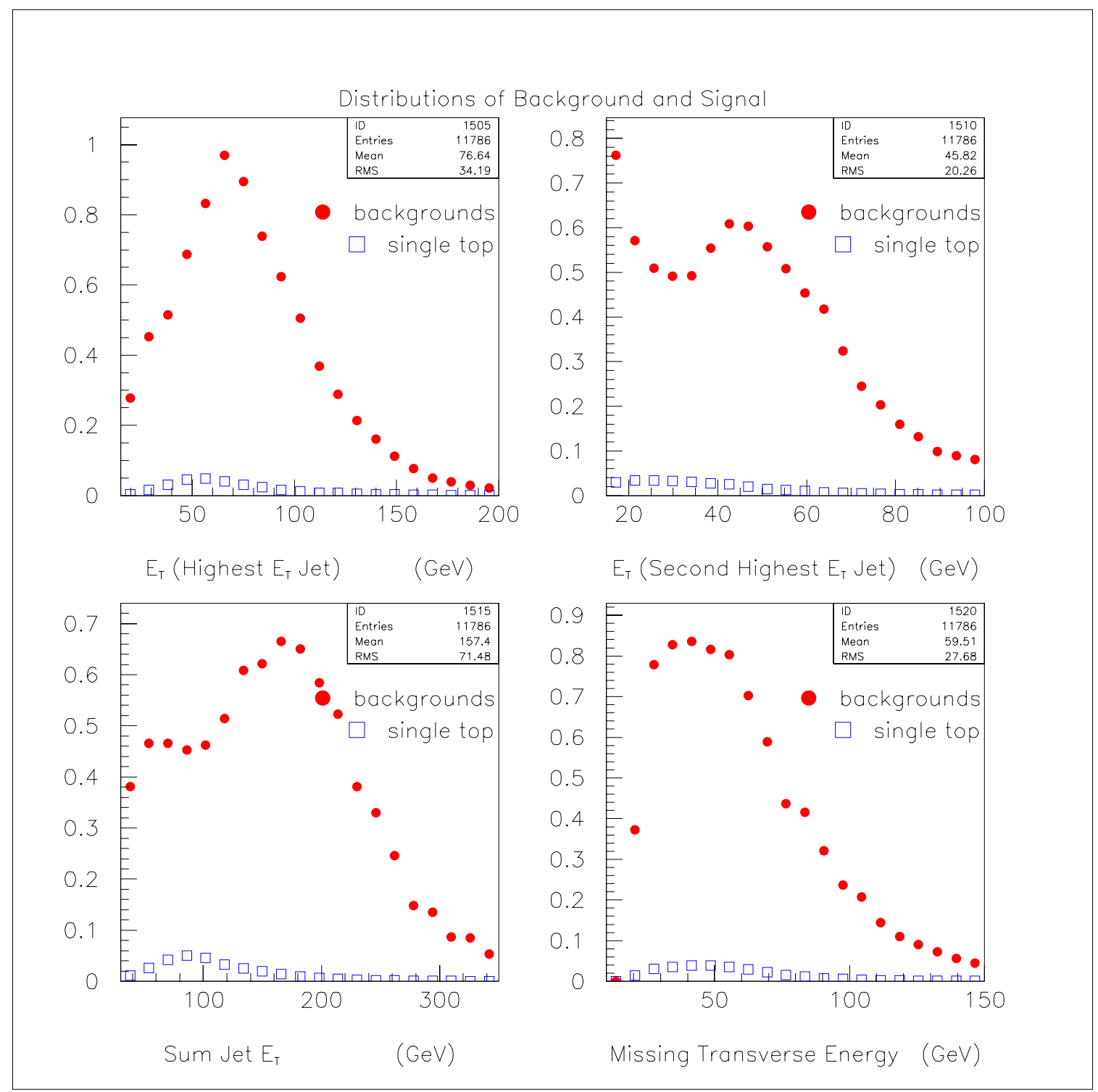

Figure 6.13. The signal and background distributions shown as functions of kinematic variables. The signal and background have been normalized to their calculated values, see Table 6.9 and Fig. 6.14. 
Table 6.9. Total number of events expected in muon + jets/tagged analysis. This is for Run IB only.

\begin{tabular}{|c|c|}
\hline Background & Expected Events \\
\hline$t \bar{t}$ & $3.2 \pm 1.1$ \\
QCD Multi-Jet & $2.2 \pm 0.6$ \\
W+jets & $2.7 \pm 0.5$ \\
\hline \hline Total & $8.1 \pm 1.3$ \\
\hline \hline Data Events & 8 \\
\hline
\end{tabular}

\subsection{Single Top Acceptances}

The geometric acceptance is determined from a sample of events where the $W$ from the single top quark is allowed to decay into either $\tau$ or $\mu$. The $\tau$ is allowed to decay however it likes. The $B R(\tau \rightarrow \mu)=17.37 \pm 0.09$ [16].

The $B R^{a c c}(b \rightarrow \mu)$ represents the quantities $\operatorname{BR}(b \rightarrow \mu)$, the branching fraction for $b$-quarks to muons, times the geometric acceptance of finding a soft muon in the detector.

$\epsilon_{\text {trig }}^{s t(2 \mu)}$ is the trigger efficiency for finding a single top event. This efficiency is determined from single top Monte Carlo events with both an isolated and tagging muon. The program TRIGSIM is used to determine the trigger efficiency.

Finally, the muon ID efficiency enters into the acceptance calculation as $\epsilon_{\mu}^{i}$ for the isolated muon and $\epsilon_{\mu}^{s}$ for the tagging muon. For Run IB muons, the weighted muon ID efficiency is $88.5 \%$. The full efficiency calculation is given by

$$
\begin{aligned}
\epsilon_{s t} & =\epsilon_{t r i g}^{s t(2 \mu)} \epsilon_{\mu}^{i} \epsilon_{\mu}^{s}, \\
A_{t b \rightarrow \mu+j e t s / \mu} & =\frac{N_{p} \epsilon_{s t}}{N_{t o t}} B R_{b \rightarrow \mu}, \\
A_{t b \rightarrow a n y} & =\frac{N_{p} \epsilon_{s t} B R(W \rightarrow \tau, \mu)}{N_{t o t}},
\end{aligned}
$$




\section{Breakdown of the 210 Single Top Events in Run IB}

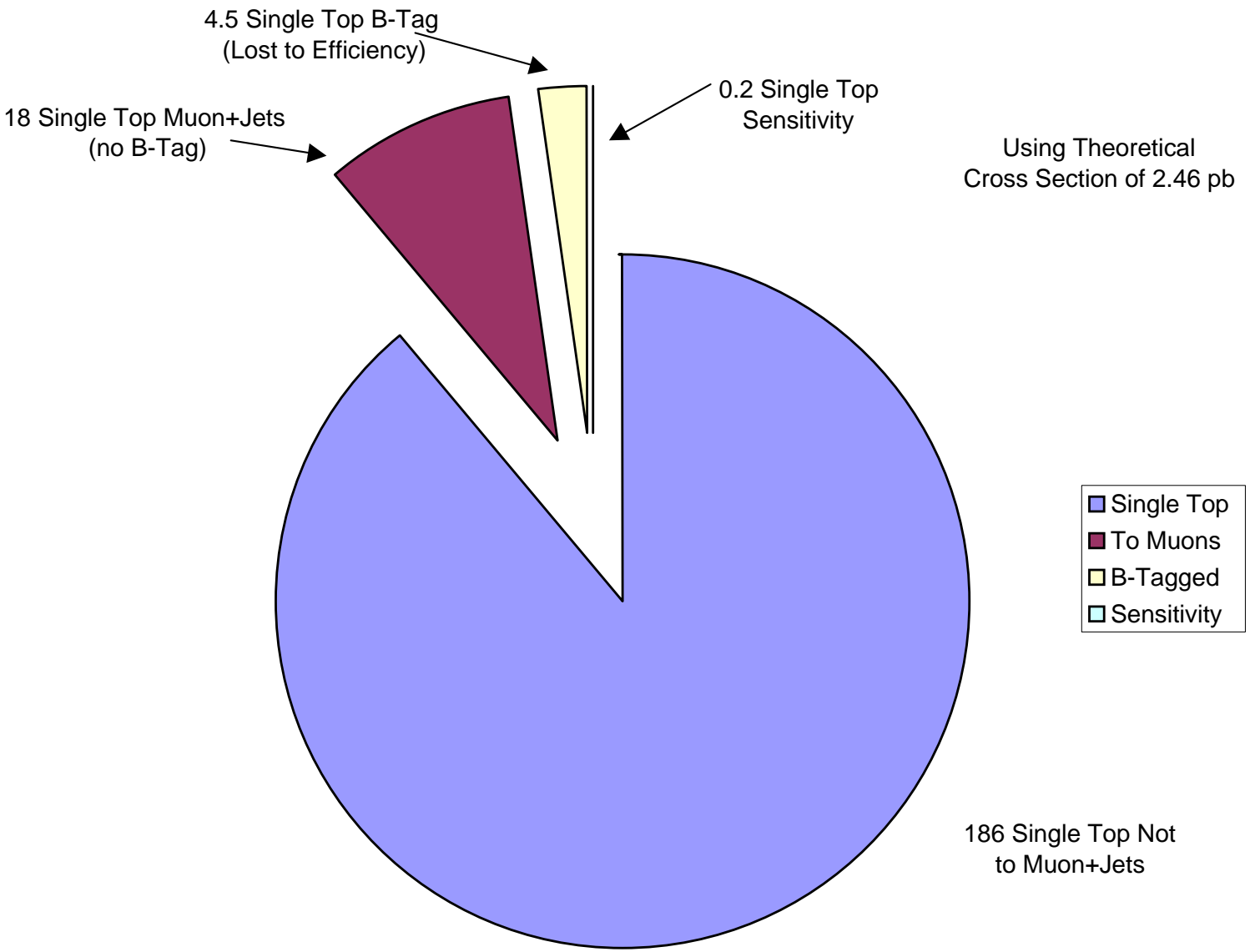

Figure 6.14. The distribution of backgrounds between QCD, $W+$ jets and Top backgrounds.

where

- $\epsilon_{s t}$ represents all efficiency factors for single top.

- $N_{p}$ is the number of Monte Carlo events passing the selection requirements (see Section 6.3).

- $N_{\text {tot }}$ is the total number of events in the Monte Carlo sample.

- $B R(W \rightarrow \tau, \mu)$ is the branching fraction for $W$ to $\mu$ or $\tau$ decay channel and is $\frac{2}{9}$. 
Table 6.10. Acceptances for single top MC without requiring the muon tag. The trigger efficiencies are for events with a tagging muon for a MU_JET_XXX filter. The MC geometric acceptances and branching fractions for tagging muons vary from $7.5 \%$ to $16.7 \%$.

\begin{tabular}{|c|r|r|r|r|r|}
\hline \multicolumn{2}{|c|}{ model/channel } & Passing Events & Geo. Acc. & Trig Eff. & $\epsilon_{\text {real }}^{\text {acc }}$ \\
\hline \multirow{2}{*}{ CompHEP } & qtb & $8005(98100)$ & $0.0816 \pm 9 \times 10^{-4}$ & $0.708 \pm 0.07$ & 0.827 \\
\cline { 2 - 6 } & tb & $8047(100000)$ & $0.0805 \pm 9 \times 10^{-4}$ & $0.724 \pm 0.07$ & 0.805 \\
\hline \multirow{2}{*}{ PYTHIA } & qtb & $958(12682)$ & $0.0755 \pm 0.0023$ & $0.708 \pm 0.07$ & 0.821 \\
\cline { 2 - 7 } & tb & $716(7410)$ & $0.0966 \pm 0.0034$ & $0.725 \pm 0.07$ & 0.792 \\
\hline
\end{tabular}

Table 6.11. The single top efficiencies for finding the tagging muon. The $\epsilon_{\text {soft }}^{\mu}$ includes all efficiencies except the eye-scan efficiency. All single top events have two $b$-quarks.

\begin{tabular}{|c|c|c|}
\hline \multicolumn{2}{|c|}{$B R(b \rightarrow \mu) \times \epsilon_{\text {soft }}^{\mu}(\%)$} \\
\hline CompHEP & qtb & $10.4 \pm 0.34$ \\
\cline { 2 - 3 } & tb & $15.7 \pm 0.41$ \\
\hline PYTHIA & qtb & $7.5 \pm 0.85$ \\
\cline { 2 - 3 } & tb & $16.75 \pm 1.4$ \\
\hline
\end{tabular}

In Eq. 6.7, the quantity $A_{t b \rightarrow \mu+j e t s / \mu}$ is the acceptance for single top to muon + jets final states with a muon tag. The quantity $A_{t b \rightarrow a n y}$ is the acceptance for single top to anything.

The $B R(b \rightarrow \mu)$ is determined from Monte Carlo events. The results vary by generator and by process. The single top process qtb has on average fewer $b$-quarks than does the $t \bar{b}$ process. This is due to the smaller geometric acceptance of the final state $b$-quark in the qtb process. There is a $28 \%$ difference between the $B R^{a c c}(b \rightarrow \mu)$ between the PYTHIA and CompHEP generators. The difference is attributed to the modeling of the spectator $b$-quark of the t-channel qtb process. 


\section{CHAPTER 7}

\section{RESULTS AND CONCLUSIONS}

\subsection{Cross Section Limits for Single Top}

The number of events we observe 8 , is consistent with our estimate of the background, $8.1 \pm 1.3$. We conclude that we have no evidence of single top production and thus, cannot provide evidence of single top production at $\mathrm{D} \varnothing$. We can, however, set an upper limit on the single top production cross section.

The cross section limits for this channel are calculated using a standard D $\varnothing$ pro-

gram, see Appendix C. The cross section limit calculator depends on the background estimate, the number of data events, signal acceptance and the luminosity, along with their respective errors.

We compare CompHEP and PYTHIA generators. The CompHEP calculation uses PYTHIA for fragmentation. We use PYTHIA as a cross check against the CompHEP calculation.

Using the efficiencies and acceptances above, we calculate the single top efficiencies for various production processes and models. With these efficiencies in hand, we can calculate the expected number of signal events and the cross section limit.

If we combine the s-channel and t-channels processes, as follows

$$
\epsilon_{t b+q t b}=\frac{\sigma_{t b} \times \epsilon_{t b}+\sigma_{q t b} \times \epsilon_{q t b}}{\sigma_{t b}+\sigma_{q t b}}
$$

where 
Table 7.1. Single top identification efficiencies for the single top to muon + jets channel with a tagging muon. The efficiencies include trigger, muon identification efficiencies and the efficiency for finding a tagging muon.

\begin{tabular}{|c|c|c|}
\hline \multicolumn{3}{|c|}{ Efficiencies $\left(\times 10^{-2}\right)$} \\
\hline CompHEP & qtb & $6.5 \pm 0.93$ \\
\cline { 2 - 3 } & tb & $10.0 \pm 1.1$ \\
\hline PYTHIA & qtb & $4.7 \pm 0.66$ \\
\cline { 2 - 3 } & tb & $10.7 \pm 1.2$ \\
\hline
\end{tabular}

Table 7.2. Single top acceptances for the muon + jets channel with a tagging muon and the acceptance for single top to any final state. The acceptances are calculated using Eq. 6.7. The two processes are weighted by the cross sections and combined, see Eq. 7.1.

\begin{tabular}{|c|c|r|r|}
\hline \multicolumn{4}{|c|}{ Acceptances $\left(\times 10^{-2}\right)$} \\
\hline Generator & Process & $\mu+j$ ets $/ \mu$ & All Final States \\
\hline CompHEP & qtb & $2.4 \pm 0.3$ & $0.12 \pm 0.02$ \\
\cline { 2 - 4 } & tb & $3.7 \pm 0.4$ & $0.18 \pm 0.02$ \\
\cline { 2 - 4 } & Both & $2.8 \pm 0.4$ & $0.13 \pm 0.02$ \\
\hline \multirow{3}{*}{ PYTHIA } & qtb & $1.6 \pm 0.2$ & $0.079 \pm 0.01$ \\
\cline { 2 - 4 } & tb & $4.7 \pm 0.5$ & $0.23 \pm 0.03$ \\
\cline { 2 - 4 } & Both & $2.5 \pm 0.3$ & $0.12 \pm 0.02$ \\
\hline
\end{tabular}

- $\epsilon_{t b+q t b}$ is total efficiency for all single top production process,

- $\sigma_{t b}$ and $\epsilon_{t b}$ are the cross section and total efficiency for the s-channel processes and

- $\sigma_{q t b}$ and $\epsilon_{q t b}$ are the cross section and total efficiency for the t-channel processes. 
Table 7.3. Cross section limits for the $\mu+$ jets $/ \mu$ channels.

\begin{tabular}{|c|c|c|c|c|c|}
\hline \multicolumn{6}{|c|}{ Run IB } \\
\hline \multirow[t]{2}{*}{ Production } & \multirow[b]{3}{*}{ Process } & \multicolumn{2}{|c|}{$\begin{array}{l}\text { Cross Section for } \\
\qquad \mu+\text { jets } / \mu\end{array}$} & \multicolumn{2}{|c|}{$\begin{array}{c}\text { Total } \\
\text { Cross Section }\end{array}$} \\
\hline & & \multicolumn{4}{|c|}{ Confidence Limit (pb) } \\
\hline Model & & $95 \%$ & $90 \%$ & $95 \%$ & $90 \%$ \\
\hline \multirow[t]{3}{*}{ CompHEP } & qtb & 4.3 & 3.4 & 85 & 69 \\
\hline & tb & 2.7 & 2.3 & 55 & 46 \\
\hline & both & 3.5 & 2.9 & 74 & 61 \\
\hline \multirow[t]{3}{*}{ PYTHIA } & qtb & 6.4 & 5.2 & 129 & 105 \\
\hline & $\mathrm{tb}$ & 2.1 & 1.8 & 43 & 36 \\
\hline & both & 3.9 & 3.2 & 82 & 67 \\
\hline
\end{tabular}

The cross section limit for single top to $\mu+$ jets $/ \mu$ channel is 3.7 (3.0) pb for the PYTHIA single top production at the 95\% (90\%) confidence level. For CompHEP, the cross section limit for single top in the $\mu+$ jets $/ \mu$ channel is $3.5(2.9) \mathrm{pb}$ at the 95\% (90\%) confidence level. The cross section limit results are summarized in Table 7.3 .

\subsection{Conclusions}

We have searched for the single top process in the $\mu+$ jets $/ \mu$ channel, but have observed no excess over the background. After all efficiencies are accounted for, we expect a single top contribution in our final event sample of less than 0.2 events. It is clear that the single top process will continue to be an elusive and challenging process to discover. However, the rewards of the study warrant our efforts to continue the search. Prospects for finding single top at the next collider run of DØ (Run II) are 
discussed in Appendix E. I am of the opinion that much remains to be improved in order to observe single top in the next run.

The single top yields suffer from the branching fraction to the $\mu+$ jets $/ \mu$ channel. In order to make precise measurement of $\left|V_{t b}\right|$ it will be necessary to use all leptonic channels of single top decay. Unfortunately, the untagged leptonic channels have much larger background than the tagged channels. Efforts are also underway to try to use the hadronic channels of single top for observation.

The study of single top in untagged leptonic channels requires a solid understanding of the $W+$ jets background. Unfortunately, the $W+$ jets background is understood only to the $30 \%$ level. A better $W+$ jets cross section calculation is desired for future studies because the single top signal is typically of the same order (or less) of the error on the $W+$ jets background calculation. The errors on the backgrounds may prevent any strong statements from being made about single top physics.

The techniques used for signal observation also have much room for improvement. This analysis used a classical analysis technique. Multivariate techniques are extremely powerful and could improve the analysis. Multivariate techniques, such as neural networks, improve the signal acceptances by optimizing the cuts. Typically, the selection criteria are loosened and the network optimizes the cuts for a given set of variables. Multivariate techniques have been used to optimize the selection cuts here, but have not been found to significantly improve the signal acceptance. To use the full power of these techniques, the signal models must accurately represent the kinematics of the single top interactions.

The modeling of single top interactions, especially the t-channel processes, were found to be different for different generators (Section 3.3). The disagreement is an indication that there is theoretical work which must be improved. I understand that an improved generator is in the final stages of development [100]. In addition, 
theoretical studies are underway to reduce the uncertainty on the single top cross section [33].

In short, much work remains to prepare for single top observation in Run II. It is my hope that the present study will be of great use in observing single top processes during Run II. 


\section{APPENDIX A}

\section{USEFUL DEFINITIONS}

The terms which are specific to High Energy Physics are defined below.

\section{A.1 High Energy Physics Specific Terms}

1. CTEQ - The Coordinated Theoretical-Experimental Project on QCD. The CTEQ group works to provide descriptions of QCD and provides useful tools to assist in the experimental study of QCD phenomena.

2. DØ - one of two collider detector experiments at Fermilab. The D $\varnothing$ experiment is so named because of its geographic location on the Fermilab accelerator ring.

3. CDF - Collider Detector Facility, CDF is the other collider detector experiment at Fermilab.

4. LO - (Leading Order), a observable is perturbatively calculated using only the primary contributions to the observables.

5. $\overline{\mathrm{MS}}$ - modified minimal subtraction scheme for renormalization.

6. NLO - (Next-to-Leading-Order), when an observable is calculated, perturbation theory is used and the results are approximate. The NLO calculation is a more accurate description which takes into account more of the perturbative contributions. 
7. PDF - (Parton Distribution Functions), when an observable calculation is performed the PDFs contain the information about the non-perturbative part of the observable calculation. The PDFs contain the relative quark flavor and gluon content of a parton at a give momentum.

8. perturbation theory - used to approximate a true observable. Since many QCD observables are not exactly calculable, perturbative theory is required to obtain an estimate of the observable.

9. renormalization - QCD theories are approximate descriptions of nature that when calculated, sometimes yield unphysical results. Renormalization removes the unphysical divergences from the theory.

DØ specific terms are listed below.

\section{A.2 DØ Specific Terms}

1. CAFIX - the DØ jet energy correction program. CAFIX corrects jets for noise, underlying events, instrumentation response, and out-of-cone energy.

2. hadronic punchthrough - this term usually refers to hadronic activity which has escaped the calorimeter. Most hadronic activity should be contained within the calorimeter however, certain regions are not well instrumented and thus it is possible for activity to pass through the calorimeter in these regions.

3. postzap - this refers to the period of D $\varnothing$ Runs after 89,000. The muon chambers were removed and "zapped" with a chemical which improved the muon system efficiencies. The deposits on the wire chambers in the muon system were removed by "zapping". 
4. prezap - this refers to the period of D $\varnothing$ Runs before 89,000. This period saw a gradual degradation of the muon system.

5. tag - an event is tagged when a muon is found within the cone of a reconstructed jet. The muon is an indication of a heavy flavor decay process and is used to reduce the backgrounds.

6. tagrate function - a function which parameterizes the probability that a calorimeter object, such as a jet, will produce a tagging muon. The tagrate function is used to calculate backgrounds which have a tagging muon. Tagrate functions may not depend on the underlying partons which give rise to a jet, such functions are called "flavor blind". Tagrate functions can also be parameterized in terms of the underlying partons. As an example, the heavy flavor tagrate function is a tagrate function for $b$-quark jets and has a higher average value than the "flavor blind" tagrate.

7. Tagrate7 - the unfortunate name of one of the tagrate function which was developed by DØ. Tagrate7 is a flavor blind tagrate function.

$\mathrm{D} \varnothing$ terms specific to the $\mathrm{D} \varnothing$ muon system are listed below.

\section{A.3 Muons Specific Terms}

1. A-stub - a muon which has hits in the A-layer but no hits in the muons system layers B or C is called an A-stub. Such "muons" do not penetrate the toroid. It is impossible to measure the momentum of an A-stub muon.

2. CF - (Central Iron) muons found in the muon system found in the central region (approximately, $|\eta|<0.95$ ). 
3. EF - (End Iron) muons reconstructed in the forward region of the muon system (approximately, $0.95<|\eta|<1.8$ ).

4. isolation - this term is applied to muons found in an event. The muon is said to be isolated if it is located outside of the jet cone in $\eta-\phi$ space. The cone size varies, but in this analysis, the jet cone is 0.5 in $\eta-\phi$ space.

5. MTC - Muon Tracks in Calorimeter, the calorimeter response is sensitive enough to determine if a minimum ionizing particle (such as a muon) has passed through it.

6. Muon Track Badness (IFW4) - A measure of the number of bad qualities of a muon track when a global fit is performed. This is an integer number representing the number of bad features of the reconstructed muon track. A value of 0 or 1 is a good globally fit muon. 


\section{APPENDIX B \\ LUMINOSITY, TRIGGERS AND VETO \\ CONDITIONS}

\section{B.1 Integrated Luminosity of the Data Sample}

A number of tools have been used to determine the luminosity for the data sample used in this analysis. The luminosity is a function of the accelerator performance, detector live-time and veto conditions implemented by the online filter.

\section{B.1.1 Triggers/filters}

Events are selected with a list of filters and veto conditions. A recorded event is the result of an event passing, for the final state objects of interest, an online trigger and a filter which depends on more detailed event information. A trigger typically is associated with a number of filters. A trigger defines a list of conditions which may cause it to "fire". The conditions required for an event to fire a trigger are simple. The level one trigger is a hardware trigger which makes a decision based on coarse calorimeter and muon information. The filter occurs at level two. A trigger can also use veto conditions, which are discussed below.

In our event selection, we require one of several filters. The choice of filters was based upon the type of events required, background contamination and the filters which give the most luminosity. 
For this analysis, we require our events to pass a MU_JET_XXX trigger/filter. The MU_JET_XXX family of filters includes, for example, MU_JET_HIGH, MU_JET_CAL, MU_JET_MAX and MU_JET_MON. The list of triggers/filters and their definitions changed frequently during a run period so, in a particular run range a given filter may have not been available. The efficiency for these triggers, which have a single isolated muon and at least one tagging muon, is about $70 \%$ for single top events. The efficiency was seen to increase only slightly by allowing additional filters. The typical event topology for single top events is an isolated muon, missing transverse energy and 2-3 jets. The set of MU_JET_XXX filters is the most appropriate for single top.

\section{B.1.2 Veto conditions}

The active main ring veto was based on three terms: the main_ring_veto_low (mr_veto_low), micro-blanking (ublank) and MRBS_Loss (mrbs). The maxi_live veto condition which would reject the fewest number of single top events requires mrbs and ublank. The goodbeam condition rejected events with the mrbs or ublank conditions. The good_cal condition rejected events with both mrbs and ublank or mr_veto_low.

Veto conditions/definitions varied during the course of Run I. A partial documentation of the history of triggers/filters for Run I can be found in Ref. [90]. We keep events which pass a goodbeam condition or events which have micro-blanking and not MRBS_Loss. The latter condition increases our acceptance slightly.

\section{B.1.3 Production Database}

Luminosity information at $\mathrm{D} \varnothing$ is stored in a relational database [89]. There are a number of utilities that can be used to calculate luminosity for a given set of runs, files and triggers/filters. However, care should be taken whenever using a general luminosity measure such as GET_FILE_LUM, which takes a list of files and 


\begin{tabular}{|l|l|l|l|l|}
\hline Run Period & Start & End & Triggers & Luminosity $\left(p b^{-1}\right)$ \\
\hline RUN IA & 50,000 & 70,000 & MU_JET_XXX & 10.1 \\
\cline { 2 - 5 } $\begin{array}{l}\text { RUN IB } \\
\text { RUN IC }\end{array}$ & 72,251 & 93,217 & MU_JET_XXX & 85.6 \\
\cline { 2 - 5 } & 94,000 & 96,972 & MU_JET_XXX & 8.505 \\
\hline
\end{tabular}

Table B.1. The luminosity calculation results for the $t \bar{t}$ mu+jets data stream. The luminosity error is $5.4 \%$. The luminosity quoted for Run IA has been subjected to a $5 \%$ loss in luminosity because of reprocessing inefficiencies.

the file type, then generates a luminosity based on the list. The GET_FILE_LUM utility is not a precision tool, but only a rough estimator of luminosity. The tool GET_FILT_LUM is requires additional information from the user making it a more precise tool.

\section{B.1.4 Luminosity Calculation}

Since this analysis uses the $\mu$-jet data stream used by the top group for the $t \bar{t} \rightarrow \mu+$ jets analysis, the luminosity calculation comes from that analysis [74]. The results are summarized in Table B.1. A list of processed data files and the GET_FILT_LUM utility were used to calculate the luminosity. The total integrated luminosity of the data set is $103.7 \pm 5.6 \mathrm{pb}^{-1}$. This total integrated luminosity includes a $5 \%$ loss in luminosity from the re-processing of Run IA data. The Run IA data was re-processed for improved muon identification. 


\section{APPENDIX C}

\section{CALCULATING LIMITS}

This analysis did not succeed in observing a single top signal. The background calculation is consistent with the observed number of events. The cross section therefore cannot be measured; but we an set an upper limit.

\section{C.1 The Steps for Calculating a Cross Section Limit}

In a counting experiment, the expression $P(k \mid \mu, I)$ is the probability of observing k events, given $\mu$ and $I ; \mu$ is the mean number of events and $I$ represents all prior information and assumptions on which the calculation is based. The probability of observing $\mathrm{k}$ events is described using a Poisson distribution

$$
P(k \mid \mu, I)=\frac{e^{\mu} \mu^{k}}{k !} .
$$

$I$ not only represents all information used for calculating $\mu$, but also the assumption that the Poisson distribution is the correct expression for the probability to observe $\mathrm{k}$ events. The expected number of events can be written as

$$
\mu=b+L \epsilon \sigma .
$$

In Eq. A-2, the number of background events is represented by $b ; L, \epsilon$ and $\sigma$ represent, respectively, the integrated luminosity, the signal efficiency and the cross section for the process. Combining equations A-1 and A-2, we arrive at Eq. A-3: 


$$
P(k \mid \sigma, L, \epsilon, b, I)=\frac{e^{-(b+L \epsilon \sigma)}(b+L \epsilon \sigma)}{k !} .
$$

Using Bayes theorem

$$
P(A \mid B C)=\frac{P(B \mid A C) P(A \mid C)}{P(B \mid C)}
$$

we can invert Eq. A-3 and ask the question: what is the probability of having a cross section $\sigma$ given that we have observed k events?

$$
\begin{aligned}
P(\sigma, L, \epsilon, b \mid k, I) & \propto P(k \mid \sigma, L, \epsilon, b, I) P(\sigma, L, \epsilon, b \mid I), \\
& \propto \frac{e^{-(b+L \epsilon \sigma)}(b+L \epsilon \sigma)}{k !} P(\sigma, L, \epsilon, b \mid I),
\end{aligned}
$$

with the normalization condition,

$$
1=\int_{0}^{\infty} d \sigma \int_{0}^{\infty} d L \int_{0}^{1} d \epsilon \int_{0}^{\infty} d b P(\sigma, L, \epsilon, b \mid k, I)
$$

The quantities $\sigma, L, \epsilon$ and $b$ are assumed to be independent of each other. This is a valid assumption for these physics processes. The prior probability $P(\sigma, L, \epsilon, b \mid I)$ therefore can be written as a product of prior probabilities,

$$
P(\sigma, L, \epsilon, b \mid I)=P(\sigma \mid I) P(L \mid I) P(\epsilon \mid I) P(b \mid I) .
$$

We assign a normalizable flat prior probability, Eq. A-8, for the cross section with $\sigma_{\max }$ chosen so that the probability that $\sigma>\sigma_{\max }$ is negligible.

$$
P(\sigma \mid I)=\left\{\begin{aligned}
\frac{1}{\sigma_{\max }} & \text { for } 0 \leq \sigma \leq \sigma_{\max } \\
0 & \text { otherwise }
\end{aligned}\right.
$$


The other prior probabilities which have been factored out of $P(\sigma, L, \epsilon, b \mid I)$ are assumed to be truncated normal distributions of the form

$$
P(\chi \mid I)=\left\{\begin{aligned}
\frac{1}{\sigma_{\chi} \sqrt{2 \pi}} e^{\frac{(\chi-\bar{\chi})}{2 \sigma_{\chi}^{2}}} & \text { if } \chi>0 \\
0 & \text { otherwise }
\end{aligned}\right.
$$

where $\sigma_{\chi}$ is the error on $\chi$ and $\bar{\chi}$ is the mean value of $\chi$.

Now that we have all the pieces, we can integrate out the nuisance (that is, uninteresting) parameters $L, \epsilon$ and $b$ in Eq. A-5,

$$
P(\sigma \mid k, I)=\int_{0}^{\infty} d L \int_{0}^{1} d \epsilon \int_{0}^{\infty} d b P(\sigma, L, \epsilon, b \mid I) .
$$

$P(\sigma \mid k, I)$ is the posterior probability distribution for the cross section for single top production given the observed event count $k$. To obtain a $95 \%$ confidence limit, Eq. A-11 must be solved for $\sigma_{U L}$ :

$$
0.95=\int_{0}^{\sigma_{U L}} d \sigma P(\sigma \mid k, I)
$$

In Eq. A-11, the quantity $\sigma_{U L}$ is called the upper limit on the cross section at $95 \%$ confidence level.

The calculation has been implemented a number of times by a number of different authors. The programs use a Monte Carlo integration technique and can take correlations among errors into account [91]. 


\section{APPENDIX D MUON + JETS/UNTAGGED ANALYSIS}

The muon+jets/untagged analysis is a precursor of the tagged analysis. The results obtained were not good enough to warrant the calculation of a limit. It is difficult to extract a single top cross section limit simply because of the large numbers of events which pass the untagged analysis cuts, the large uncertainty in the background and the statistical fluctuations. For instance with the selection requirements below, approximately 200 events are observed. The uncertainty in the background calculation is approximately $25 \%$. The expected single top signal is approximately 2 events after applying all analysis cuts. With an error of 40-50 events in the background calculation, it is extremely difficult to make meaningful statements about the single top contribution in the data sample.

This section is included in order to describe the backgrounds, selection process and to illustrate one method for normalizing the $W+$ jets background to the data.

\section{D.1 Muon+Jets (no $\mu$ tag) Analysis}

\section{D.1.1 Basic Selection Requirements}

- One Isolated Muon and trigger requirements (see Section 6.3)

- Good jets and Main Ring veto (see Section 5.2.4 and Appendix B)

- $\mathbb{E}_{T}^{c a l} \geq 15 \mathrm{GeV}$

- $\mathbb{E}_{T} \geq 20 \mathrm{GeV}$ 
- $\mathrm{N}_{j e t s} \geq 2, \mathrm{~N}_{j e t s} \leq 5$

- $\left.p_{T j e t 1} \geq 20 \mathrm{GeV},|\eta|<2.0, p_{T j e t 2} \geq 15 \mathrm{GeV},|\eta|<2.0\right)$

- no tagged muons

\section{D.1.2 QCD Multi-Jet Background to the Muon+Jets Analysis}

The QCD multi-jet background for $\mathrm{N}$ jets is calculated by multiplying the number of N+1-jet non-isolated muon events which satisfy all analysis requirements by the probability that a non-isolated muon+jet system appears as a isolated muon. Non-isolated muons statisfy all isolated muon requirements except that they are within $\Delta \mathrm{R}<0.5$ of a jet. Such events arise dominantly from heavy flavor QCD processes. The QCD multi-jet background is a strong function of the selection criteria. The QCD multi-jet background was required to meet some minimal selection requirements and pass some MU_JET_XXX filter.

We use a background sample of events for calculating the QCD background. The background sample required only one or more loose, non-isolated muons and one or more energy corrected jets, $|\eta|<2.5$ and $p_{T}>10 \mathrm{GeV}$. The probability that a non-isolated muon+jet system appears as an isolated muon is known as the false isolation probablility. The false isolation probability is the ratio of the number of isolated muon events with $\mathrm{N}$ jets and $\mathscr{E}_{T}<20$ to the number of non-isolated muon events with $\mathrm{N}+1$ jets and the $\mathscr{E}_{T}<20$. The QCD background is tabulated for EF $+\mathrm{CF}, \mathrm{CF}$ and $\mathrm{EF}$. The weighted error for the QCD background is $9.6 \%$. 

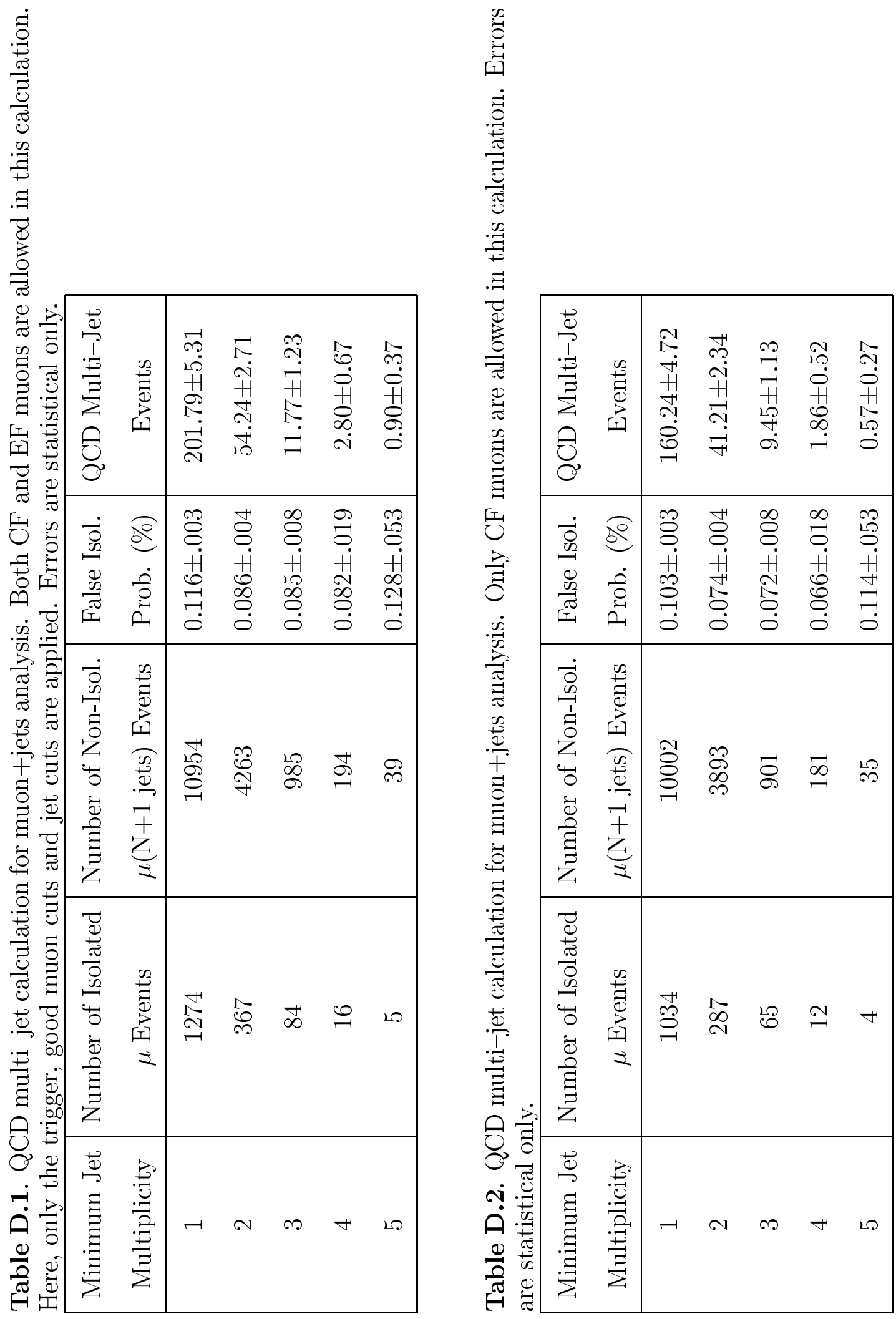


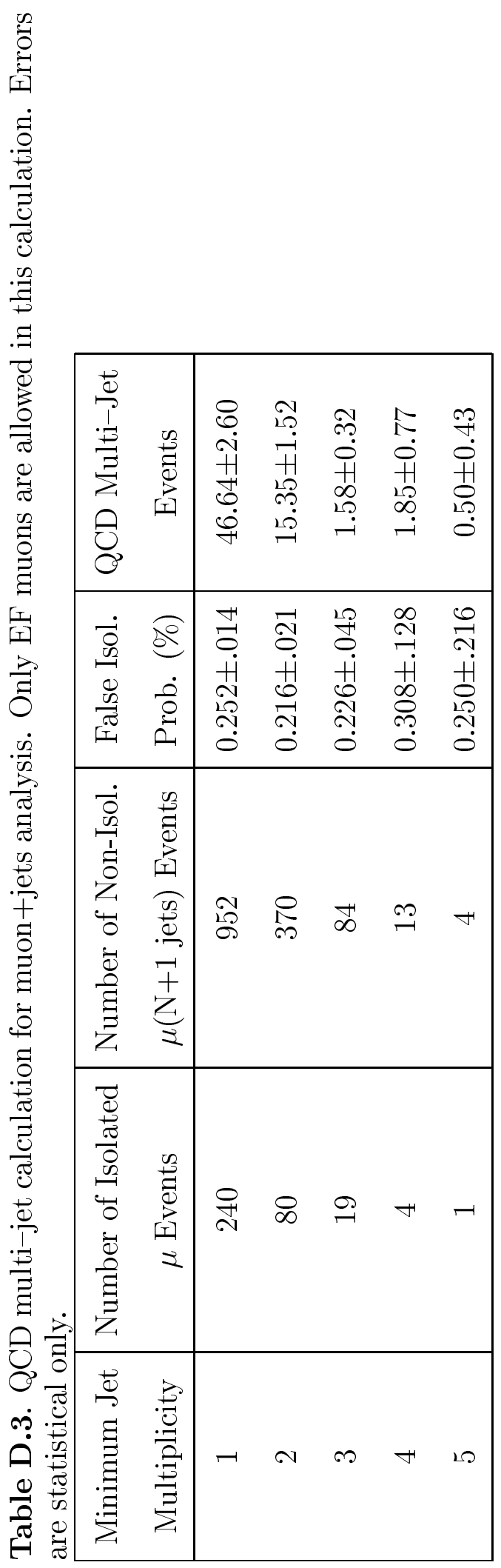




\begin{tabular}{|c|c|c|c|c|}
\hline $\begin{array}{c}\text { Minimum Jet } \\
\text { Multiplicity }\end{array}$ & $\begin{array}{c}\text { Number of } \\
\text { Events }\end{array}$ & $\begin{array}{c}\text { Number of QCD } \\
\text { Events }\end{array}$ & $\begin{array}{c}\text { Total - } \\
\text { QCD }\end{array}$ & $\begin{array}{c}\text { Number of } \\
\text { W+jets }\end{array}$ \\
\hline 1 & 1170 & $201.79 \pm 5.31 \pm 60.3$ & $969 \pm 5 \pm 56$ & 1308 \\
2 & 344 & $54.24 \pm 2.71 \pm 16.3$ & $290 \pm 3 \pm 16$ & 329 \\
3 & 75 & $11.77 \pm 1.23 \pm 3.5$ & $70 \pm 1 \pm 4$ & 70 \\
4 & 23 & $2.80 \pm 0.67 \pm 0.84$ & $20 \pm 1 \pm 1$ & 20 \\
5 & 4 & $0 \pm 0.50 \pm 0.15$ & $4 \pm 2 \pm 0.6$ & 4 \\
\hline
\end{tabular}

\section{D.1.3 $W+$ jets background}

The $\mathrm{W}+$ jets background calculation is computed for $\mathrm{W}+$ jets with low multiplicity by assuming that the data can be entirely modeled by QCD and W+jets and that the $\mathrm{W}$ background at $\mathrm{N}$ jets is determined by the $\mathrm{W}$ background at 1 jet.

To compute the $\mathrm{W}+$ jets background, we use the QCD prediction and the number of events seen in the data sample. For low jet multiplicity there is a trigger inefficiency. For the MU_JET_XXX trigger, we correct the one-jet multiplicity by $1.35 \pm 0.026$. For the two-jet case, we correct by $1.135 \pm 0.032$. For three-jets, the correction is $1.087 \pm 0.064[74]$.

\section{D.1.4 tt $t \bar{t}$ Backgrounds}

Another primary contribution to the single top background is $t \bar{t}$ events. $t \bar{t}$ events have similar signature to single top events. However, $t \bar{t}$ events tend to be more energetic than single top events and also tend to have more jets. In order to keep as much single top acceptance as possible, we allow these energetic events and events with up to five jets.

We calculate the $t \bar{t}$ background using the D $\varnothing$ cross-section, and acceptances based on $t \bar{t}$ Monte Carlo events. We use the $\mathrm{D} \emptyset 5.5 \pm 1.8 \mathrm{pb}$ cross section, trigger efficiency 
$73.7 \% \pm 7.3 \%$, isolated muon ID efficiency of $94.1 \% \pm 1.1 \%$. We find the acceptance for $t \bar{t}$ pair production to be $0.0974 \pm 0.0098$ from Monte Carlo studies.

Using the above numbers, we can calculate the $t \bar{t}$ background in $85.6 \mathrm{pb}^{-1}$ $( \pm 5.4 \%)$. We find the $t \bar{t}$ contribution to the single top muon + jets untagged analysis to be $11.38 \pm 1.52$ events. 


\section{APPENDIX E SINGLE TOP SENSITIVITY IN RUN II}

With the expected high luminosity at theTevatron, we will be sensitive to single top production in Run II. We describe the predicted sensitivity to single top in Run II and beyond. These results were presented at the 1998 Top Thinkshop, at Fermilab.

\section{E.1 Cross Sections}

In addition to increases in luminosity, one expectes an increase of the center of mass energy to $\sqrt{s}=2.0 \mathrm{TeV}$ at the Tevatron. The cross sections for single top are predicted to increase with $\sqrt{s}$.

The s-channel single top cross section is expected to increase from its value at $\sqrt{s}=1.8 \mathrm{TeV}$ by $22 \%$ to $0.894 \pm 0.064 \mathrm{pb}$. This cross section was calculated at next-to-leading order (NLO) using $m_{t}=175 \mathrm{GeV}$ and the CTEQ4M parton distribution functions [34]. The t-channel process is expected to increase by $44 \%$ to $2.44 \pm 0.27 \mathrm{pb}$. The cross section was calculated at NLO with $m_{t}=175 \mathrm{GeV}$ and the CTEQ4M parton distribution functions [32].

\section{E.2 Previous Studies}

A previous study of single top is available from [92] which relies on the TeV-2000 Report [93]. The results from this study will be summarized here.

The study reports that the primary backgrounds are $W+$ jets production and $t \bar{t}$ backgrounds. The study uses the ONETOP [94] Monte Carlo program for single top 
simulations. For $\mathrm{m}_{t}=180 \mathrm{GeV}, \sqrt{s}=2.0 \mathrm{TeV}, \int \mathcal{L}=2 \mathrm{fb}^{-1}$, the study finds a signal acceptance of 0.20 with a b-tag ${ }^{1}$, with an error of $7 \%$. The signal to background ratio is $1: 2$, with 650 events expected. Both $\mu+$ jets and $e+$ jets channels are used.

The study claims that a total error on the single top cross section of $10 \%$ statistical and $16 \%$ systematic can be achieved in Run II with $2 \mathrm{fb}^{-1}$ of luminosity. The total error on $\left|V_{t b}\right|$ was determined to be $12 \%$.

\section{E.3 Current Projections}

With more statistics and more accurate event generators to model the backgrounds and signals, the $\mathrm{TeV} 2000$ study for single top was recalculated using the improved generators and a more realistic background model.

\section{Acceptance for Single Top}

At the time of this single top study, three distinct diagrams for t-channel single top production were considered. As a result of the conference, it was noted that there are in principle only two t-channel diagrams; the third is unphysical.

In Table E.1, $g q \rightarrow t q b$ and $q b \rightarrow t q$ should be added together to obtain results for the t-channel cross section.

The acceptances are based on loose requirements for a lepton $(e$ or $\mu)$ and two or three jets. The first jet must have transverse energy greater than $15 \mathrm{GeV}$, the second and third jets must have transverse energy greater than $10 \mathrm{GeV}$; all jets must have $|\eta|<2.5$.

In Table E.1, acceptances for all-hadronic modes of signal and background production are displayed for all modes except QCD backgrounds. The QCD background is expected to dominate all signal and other background processes. With

\footnotetext{
${ }^{1}$ This assumes an upgraded $\mathrm{D} \varnothing$ detector with a silicon vertex detector with $50 \%$ efficiency for tagging a b-quark.
} 
$t \bar{t}$ production, it was extremely difficult to extract a $t \bar{t}$ all-hadronic signal [95]. It is expected to be even more difficult with single top because of the lower jet multiplicity and softer jets. Nevertheless, with an upgraded DØ detector and a silicon tracker trigger, which will tag heavy flavor events, it may be possible to extract a signal from single top in the all hadronic channel.

\section{E.4 Event Yields}

The single top event yields (Table E.2) have been calculated based on the acceptances in Table E.1. It is expected, in the single top lepton+jets channel, that there will be approximately 160 single top events and 1262 background events. The signal to background would be 0.13 .

The above calculation was based on a number of assumptions about the upgraded $\mathrm{D} \varnothing$ detector and the silicon tracker trigger performance. The efficiency for tagging a $b$-quark was taken to be $55 \%$. This included both soft lepton tagging and tagging using a displaced vertex in the silicon detector. There is a non-zero chance that a $c$-quark or some other light quark will be mistaken as a $b$-quark. The mistag rate for $c$-quarks was taken to be $15 \%$ and the mistag rate for any light quark was taken to be $0.4 \%$. These tag rates are roughly based on the experience from CDF's silicon tracking system.

In addition, the particle identification efficiency was taken to be $70 \%$ and the trigger efficiency was taken to be $90 \%$. For all-hadronic channels, the silicon tracker trigger was assumed to have an efficiency of $77 \%$.

\section{E.5 Single Top Sensitivity in Run II}

In order to display our sensitivity for finding a signal and making an actual measurement of cross section and $\left|V_{t b}\right|$, the expected errors on the cross section and 
$\left|V_{t b}\right|$ have been calculated based on the single top yields and acceptances. We shall take the relative error $\frac{\Delta V_{t b}}{\left|V_{t b}\right|}$ as a measure of sensitivity of $\left|V_{t b}\right|$. If the same errors from Heinson et al. [92] are assumed then, for 2-3 jets, the experimental error on the cross section is $15 \%$ (stat) and $53 \%$ (sys) for $2 \mathrm{fb}^{-1}$ with silicon tagging and soft lepton tagging of $b$-quarks. The total error on the cross section is $56 \%$.

The relative error in $\left|V_{t b}\right|$ is half that of the cross section and is calculated to be $28 \%$ (including systematic and statistical errors).

In order to be more sensitive to single top events at the Tevatron, the single top group must be very careful to choose methods which minimize the systematic error for finding single top. It is expected that with time the methods will improve and to this end, the results predicted here will be drastically improved. 


\begin{tabular}{|c|c|c|c|c|c|c|c|c|c|c|c|}
\hline 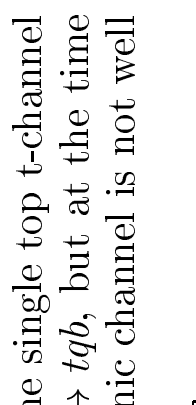 & & & & & & & & & & & \\
\hline 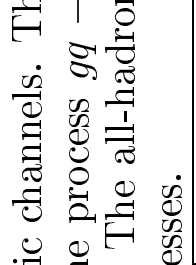 & 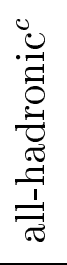 & $\stackrel{\infty}{\stackrel{\infty}{\ominus}}$ & $\stackrel{\sim}{\stackrel{1}{0}}$ & $\stackrel{\stackrel{10}{\Upsilon 1}}{0}$ & $\begin{array}{l}+1 \\
\stackrel{0}{ } \\
\vec{x} \\
\infty\end{array}$ & 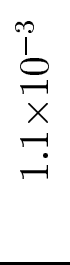 & $\stackrel{F}{\circ}$ & $\stackrel{\infty}{0}$ & 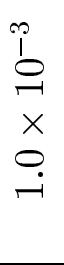 & $\begin{array}{l}\infty \\
0 \\
0 \\
x \\
0 \\
i\end{array}$ & 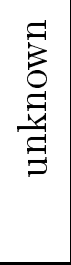 \\
\hline 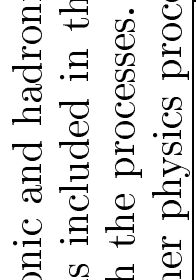 & 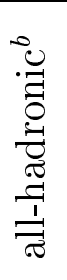 & $\stackrel{?}{\circ}$ & $\stackrel{\infty}{\stackrel{1}{0}}$ & $\stackrel{\leftrightarrow}{\stackrel{\circ}{0}}$ & $\begin{array}{l}\infty \\
i \\
o \\
\vec{x} \\
\underset{\sim}{-} \\
\vec{r}\end{array}$ & 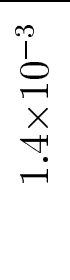 & $\stackrel{10}{\stackrel{10}{0}}$ & 옹 & 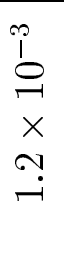 & 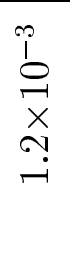 & 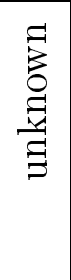 \\
\hline 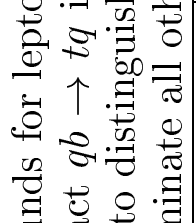 & 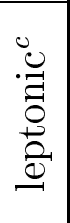 & $\stackrel{n}{\stackrel{2}{0}}$ & 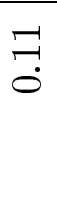 & $\stackrel{10}{\stackrel{10}{0}}$ & $\begin{array}{c}+1 \\
\dot{0} \\
\stackrel{0}{x} \\
\dot{x} \\
0 \\
\dot{0}\end{array}$ & 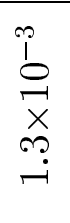 & $\stackrel{1}{\circ}$ & $\stackrel{10}{\circ}$ & $\begin{array}{l}\qquad \\
1 \\
0 \\
\ddots \\
x \\
0 \\
0\end{array}$ & $\begin{array}{l}+1 \\
0 \\
0 \\
\times \\
0 \\
0\end{array}$ & $\begin{array}{l}0 \\
\stackrel{0}{0} \\
\stackrel{g}{g}\end{array}$ \\
\hline 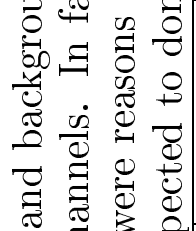 & $\begin{array}{l}0 \\
.0 \\
0 \\
0 \\
0 \\
0 \\
0 \\
0\end{array}$ & $\stackrel{\infty}{\stackrel{\infty}{0}}$ & ન્ન & $\stackrel{0}{\circ}$ & $\begin{array}{c}+1 \\
b \\
0 \\
\times \\
x \\
0 \\
1\end{array}$ & 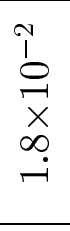 & $\stackrel{8}{\stackrel{0}{0}}$ & $\stackrel{\leftrightarrow}{\varrho}$ & $\begin{array}{l}3 \\
1 \\
0 \\
+1 \\
x \\
0 \\
1\end{array}$ & $\begin{array}{l}+1 \\
1 \\
0 \\
\times \\
\times \\
0 \\
1\end{array}$ & 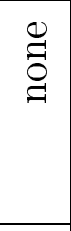 \\
\hline 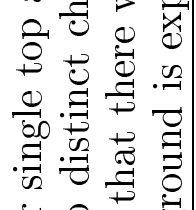 & 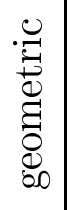 & $\stackrel{\leftrightarrow}{\leftrightarrow}$ & $\stackrel{\leftrightarrow}{\circ}$ & $\stackrel{\leftrightarrow}{\leftrightarrow}$ & $\stackrel{\Omega}{\stackrel{\Omega}{0}}$ & 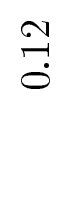 & $\stackrel{\rho}{\stackrel{\leftrightarrow}{\circ}}$ & $\stackrel{\overbrace{}}{\overparen{\leftarrow}}$ & $\stackrel{\infty}{\overparen{0}}$ & $\stackrel{\infty}{\stackrel{0}{0}}$ & 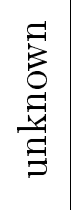 \\
\hline 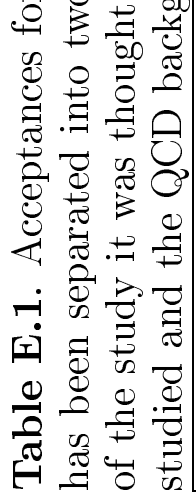 & $\begin{array}{l}0 \\
0 \\
0 \\
d \\
0 \\
0 \\
: \\
0\end{array}$ & $\begin{array}{l}\approx \\
\uparrow \\
\uparrow \\
-2\end{array}$ & $\begin{array}{l}\stackrel{o r}{+} \\
\uparrow \\
\approx\end{array}$ & $\begin{array}{l}\text { 엄 } \\
\uparrow \\
\uparrow \\
\text { or }\end{array}$ & 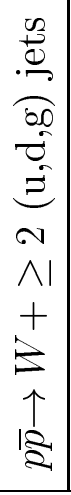 & 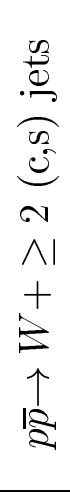 & 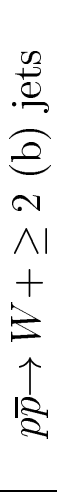 & 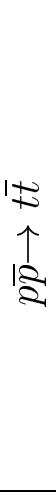 & 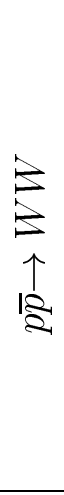 & $\begin{array}{l}N \\
\stackrel{N}{=} \\
\mathbb{2}\end{array}$ & 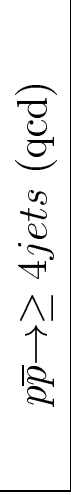 \\
\hline
\end{tabular}

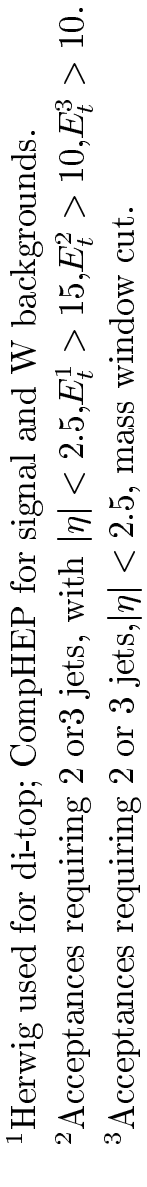


Table E.2. Events yields for single top events for signal and backgrounds to any decay channel, to leptonic decay channels and all-hadronic decay channels. The yield is calculated per $\mathrm{fb}^{-1}$ of luminosity.

\begin{tabular}{|c|r|r|r|}
\hline \multirow{2}{*}{ process } & \multicolumn{3}{|c|}{ Event Yields $/ \mathrm{fb}^{-1}$} \\
\cline { 2 - 4 } & All-Channels & leptonic & All-Hadronic \\
\hline$q q^{\prime} \rightarrow t b$ & 290 & 53 & 176 \\
\hline$q b \rightarrow t q$ & 359 & 37 & 128 \\
\hline$g q \rightarrow t q b$ & 460 & 74 & 244 \\
\hline$W+2(\mathrm{u}, \mathrm{d}, \mathrm{g})$ jets & 360,000 & 380 & 836 \\
\hline$W+2(\mathrm{c}, \mathrm{s})$ jets & 10,300 & 290 & 645 \\
\hline$W+2(\mathrm{~b})$ jets & 2700 & 386 & 850 \\
\hline$p \bar{p} \rightarrow t \bar{t}$ & 3200 & 198 & 325 \\
\hline$p \bar{p} \rightarrow W W$ & 5,000 & 7 & 12 \\
\hline$p \bar{p} \rightarrow W Z$ & 740 & 1 & 3 \\
\hline$p \bar{p} \rightarrow \geq 4 j e t s(\mathrm{qcd})$ & $1.4 \times 10^{7}$ & none & Large \\
\hline
\end{tabular}




\section{BIBLIOGRAPHY}

[1] A. Einstein, Private Conversation.

[2] E. Fermi, Ric. Sci. 4, 491 (1933). [English translation: F. L. Wilson, Am. J. Phys.36, 1150 (1968)]

[3] CDF Collaboration, F. Abe et al., Phys. Rev. Lett. 74, 2626 (1995)

[4] DØ Collaboration, S. Abachi et al., Phys. Rev. Lett. 74, 2632 (1995.)

[5] C. Quigg, "Gauge Theories of the Strong, Weak and Electromagnetic Interactions," Addison-Wesley, Reading, Mass, 1983.

[6] O. Nachtmann, "Elementary Particle Physics: Concepts and Phenomena," Springer-Verlag, New York, 1989.

[7] M. E. Peskin and D. V. Schroeder, "An Introduction to Quantum Field Theory," Addison-Wesley, Reading, Mass., 1995.

[8] D. H. Perkins, "Introduction to High Energy Physics," Addison-Wesley, Reading, Mass, 1987.

[9] R. Brock et al. [CTEQ Collaboration], "Handbook of perturbative QCD: Version 1.0," Rev. Mod. Phys. 67, 157 (1995).

[10] S. Catani, M. Ciafaloni and F. Hautmann, "High-energy factorization in QCD and minimal subtraction scheme," Phys. Lett. B307, 147 (1993).

[11] A. Salam, "Gauge Unification Of Fundamental Force," Rev. Mod. Phys. 52, 525 (1980).

[12] S. Weinberg, Science 210, 1212(1980).

[13] A. Salam, Science 210, 1114(1980).

[14] M. Kobayashi and T. Maskawa, Prog. Theor. Phys. 49, 652 (1973)

[15] G. F. Tartarelli, "Direct Measurement of $\left|V_{t b}\right|$ at CDF," Fermilab-CONF-97/401E, to appear in the European Physical Society Conference on High Energy Physics, Jerusalem, August, 1997 (unpublished). 
[16] Particle Data Group, "Review of Particle Physics," European Physical Journal C3, 1 (1998).

[17] L.M. Lederman, "The discovery of the Upsilon, bottom quark, and B mesons," In *Stanford 1992, The rise of the standard model* 101-113.

[18] C.R. Schmidt and M.E. Peskin, Phys. Rev. Lett. 69, 410 (1992).

[19] P. Grannis, plenary talk at the International Conference on High Energy Physics, Warsaw, 1996, reporting the analysis of the DØ and CDF collaborations.

[20] V. Barger, H. Baer and K. Hagiwara, Phys. Lett. 146B, 257 (1984)

[21] V. Barger and R. Phillips, "Collider Physics," Addison-Wesley, Reading, Mass., 1987.

[22] D. Carlson and C.-P. Yua, Phys. Lett. 306, 386 (1993.)

[23] D. Carlson, E. Malkawi, and C.-P. Yuan, Phys. Lett. 337, 145 (1994.)

[24] D. Atwood, S. Bar-Shalom, G. Eilam, and A. Soni, Phys. Rev. D 54, 5412 (1996.)

[25] A. Datta and X. Zhang, Phys. Rev. D 55, 2530 (1997.)

[26] C. S. Li, R. Oakes, and J. M. Yang, Phys. Rev. D 55, 1672 (1997;)Phys. Rev. D 55, 5780 (191997.)

[27] E. Simmons, Phys. Rev. D 55, 5494 (1997.)

[28] A. Datta, J. Yang, B.-L. Young, and X. Zhang, Phys. Rev. D 56, 3107 (1997.)

[29] S. Willenbrock and D. Dicus, Phys. Rev. D 34, 155 (1986)

[30] C.-P. Yuan, Phys. Rev. D 41, 42 (1990)

[31] R. K. Ellis and S. Parke, Phys. Rev. D 46, 3785 (1992)

[32] T. Stelzer, Z. Sullivan and S. Willenbrock, Phys. Rev. D 55, 5919 (1997.)

[33] Z. Sullivan, Private correspondence.

[34] M. Smith and S. Willenbrock, Phys. Rev. D 54, 6696 (1996.)

[35] E.L. Berger and H. Contopanagos, Phys. Lett. B361, 115 (1995).

[36] E. E. Boos, et al., "CompHEP - Specialized package for automatic calculations of elementary particle decays and collisions," hep-ph/9503280, 1997. 
[37] P. A. Baikov, et al., "Physical Results by means of CompHEP," hepph/9701412v2, 1997.

[38] T. Sjöstrand, Computer Physics Commun. 82(1994) 74.

[39] J. E. McDonald, at the Top Thinkshop: Top-quark Physics at Run II, at Fermilab, Oct. 1998.

[40] T. Sjöstrand, "Phythia 5.7 and Jetset 7.4: Physics and Manual," LU TP 92-20, revision of CERN-TH.7112/93.

[41] Howard Baer, Private conversation.

[42] A. Heinson, Private correspondence.

[43] Zack E. Sullivan, Private correspondence.

[44] A. Czarnecki and K. Melnikov, "Two loop QCD corrections to top quark width," hep-ph/9806244.

[45] N. G. Deshpande, B. Margolis and H. D. Trottier, Phys. Rev. D 45, 178 (1992)

[46] D. Atwood, A. Aeppli and A. Soni, Phys. Rev. Lett. 69, 2754 (1992)

[47] D. Atwood, A. Kagan and T. G. Rizzo, Phys. Rev. D 52, 6264 (1995.)

[48] C.-S. Huang and T.-J. Li, Z. Phys. C68,319 (1995).

[49] K. Cheung, Phys. Rev. D 53, 3604 (1996)

[50] J. Berger, A. Blotz, H.-C. Kim and K. Goeke, Phys. Rev. D 54, 3598 (1996)

[51] T. Tait and C.-P. Yuan, Phys. Rev. D 55, 7300 (1997)

[52] C. T. Hill, Phys. Rev. Lett. 266, 419 (1991)

[53] C. T. Hill and S. J. Parke, Phys. Rev. D 49, 4454 (1994)

[54] E. Eichten and K. Lane, Phys. Lett. B 327, 129 (1994).

[55] L. L. Smith, P. Jain and D. W. McKay, Proceedings of the 9th Annual American Physical Society Division of Particles and Fields Meeting, Minneapolis, MN, (August 1996).

[56] A. Datta and X. Zhang, Phys. Rev. D 55, 2530 (1997.)

[57] E. H. Simmons, Phys. Rev. D 55, 5494 (1997.)

[58] C.-X. Yu and G.-R. Lu, Chin. Phys. Lett. 15, 631 (1998). 
[59] D. O. Carlson and C.-P. Yuan, Particle Phys. \& Phen. 172,(1995).

[60] R. J. Oakes et al.., Phys. Rev. D 57, 534 (1998)

[61] S. Bar-Shalom, D. Atwood, and A. Soni, Phys. Rev. D 57, 195 (1998.)

[62] A. Datta, J. M. Yang, B.-L. Young and X. Zhang, Phys. Rev. D 56, 3107 (1997.)

[63] M. Abolins et al., Nucl. Instr. and Methods, A280, 36 (1989).

[64] S. Abachi et al.(DØ Collaboration), Phys. Rev. D 52, 4877 (1995.)

[65] J. Linnemann, in "Proceedings of the American Physical Society Division of Particles and Fields Conference," Fermilab (Nov. 1992), ed. R. Raja and J. Yoh.

[66] M. Abolins et al., IEEE Trans. Nuc. Sci. 36, No. 1 (1989).

[67] M. Abolins et al., IEEE Trans. Nuc. Sci. 38 (1981).

[68] R. Brun and F. Carminati, CERN Program Library Long Writeup W5013, 1993 (unpublished).

[69] W. G. D. Dharmaratna et al., DØ Note 1730, May 1993 (unpublished)

[70] The MUSMEAR program was written by T. Diehl and P. Quintas.

[71] M. Goosen et al., ZEBRA, CERN Program Library Number Q100 (1992).

[72] B. Abbot et al., "Jet Energy Scale at D0," DØNote 3287, Aug., 1997.

[73] E. Gallas, D0 note on MTC (Muons in Calorimeter).

[74] J. M. Butler et al., "Measurement of the Top Quark Production Cross Section Using lepton+jets events,", DØNote 2978, Dec, 1998.

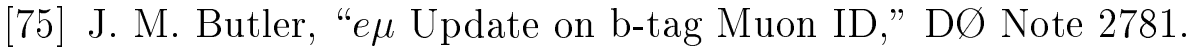

[76] D. Hedin, "Muon ID in the Overlap Region," DØNote 2757, Oct., 1995.

[77] D. Hedin et al., "Run Ib Muon Event Scanning," DØnote 2716, Sept., 1995.

[78] J. M. Butler, "EF Muon ID for b-Tagging in the Post-Zap Era," DØNote 2602, June, 1995, Revised Aug., 1995.

[79] T. Hu et al., "Offline Muon Identification in Run Ib," DØNote 2506, March 1995. 
[80] G. Marchesini et al., "Herwig: A Monte Carlo event generator for simulating Hadron Emission Reaction with Interfering Gluons," Computer Phys. Commun. 67 (1992) 465.

[81] D. Chakraborty, Ph.D. Thesis, State University of New York at Stony Brook, 1994.

[82] E. Varnes, Ph.D. Thesis, University of California at Berkeley, 1997.

[83] S. Snyder, Ph.D. Thesis, State University of New York at Stony Brook, 1995.

[84] W. T. Giele, E. Glover and D. Kosower, Nucl. Phys., B403,633,(1993).

[85] W. G. Cobau et al., "Soft $\mu$ Tagging of Jets in Multi-Jet Events,", DØNote 2863, Nov., 1996.

[86] P. Bhat, H. B. Prosper and S. Snyder, "Top Quark Physics at the Tevatron," Intl. Jour. of Mod. Phys., A 13, 5113-5218, (1998).

[87] T. Hebert, Private Conversation.

[88] John Hobbs, DØNote 2775A

[89] Laura Paterno, "DØ Luminosity Utility," Available on http://www-d0.fnal.gov/offline/pdb/lum_util.html.

[90] Mike Tartaglia, "Run 1 Trigger Lists Overview," Available on http://www-d0.fnal.gov/online/GM/trigger_lists.html

[91] J. Hobbs, Private Conversation.

[92] A. P. Heinson, A. S. Belyaev and E. E. Boos, Phys. Rev. D 56, 3114 (1997.)

[93] D. Amidei and R. Brock, ed., "Future Electro Weak Physics at the Fermilab Tevatron:Report of the tev_2000 Study Group," Fermilab-Pub-96/082.

[94] D. O. Carlson and C.-P. Yuan, Phys. Rev. Lett. 306, 386 (1993.)

[95] B. Abbot et al., "Measurement of the Top Quark Pair Production Cross Section in $\bar{p} p$ Collisions using Multijet Final States.", Accepted by Phys. Rev. D, Fermilab-Pub-98/130-E.

[96] J. M. Butler, "EF Muon ID for b-Tagging in the Post-Zap Era" DØ Note 2606.

[97] J. Hobbs, Private correspondence. 
[98] P. Quintas, "Reconstruction and Selection Efficiencies for High $p_{T}$ Muons in Run IB," DØNote 2865 and J. Butler, "Update on b-Tag Muon ID,", DØNote 2781.

[99] L. Babukhadia et al., "Hot Cells and Jets at DØ (AIDA Cell Restoration)", DØNote 3408, April, 1998.

[100] S. Mrenna, Private correspondence. 


\title{
BIOGRAPHICAL SKETCH
}

\author{
Jeffrey E. McDonald
}

\begin{abstract}
Education and Career Record
M.S., Physics, Florida State University (1996).
\end{abstract}

Teaching Assistant, Florida State University, January 1995 to May 1995.

B.S., cum laude, senior departmental honors, Physics, Clemson University (1994).

Research Aide, Clemson University, May 1993 to August 1993.

Research Aide, Clemson University, May 1992 to August 1992.

\section{Physics Research Interests}

\section{Single Top Physics}

My current research interests include the search for top quark production via the weak interactions in which a single top quark is produced in the final states. Such reactions are very interesting because they allow the investigation of weak interaction phenomena that cannot be studied using reactions that produce a $t \bar{t}$ pair via the strong interactions. At the Tevatron accelerator, a $t \bar{t}$ pair is a rare event, but single top reactions are even rarer. The cross section for single top production is about half that for $t \bar{t}$ production. Single top events are typically less energetic than $t \bar{t}$ events; they are, therefore, more difficult to detect. A major focus of my research is to study the use of multivariate methods to more efficiently identify single top quark events. I intend to use both neural network and random grid search techniques and capitalize on the work I have done looking for $t \bar{t}$ events, which contain only jets in the final states.

\section{Top Physics in the Alljets Channel}

My first major research effort, for DØ (one of the two large collider physics collaborations based at the Fermi National Accelerator Laboratory), was to devise 
methods and physics variables to aid in the search for $t \bar{t}$ production in the purely hadronic channel. In these $t \bar{t}$ reactions the top and anti-top decay $45 \%$ of the time into jets (in the ideal case, six jets). Searching for these events was a huge challenge given the enormity of the background from QCD multi-jet production. The signal to noise ratio is about $1 / 1000$. To help combat this background I helped to develop a few analysis tools, such as the random grid search and a program to generate automatically functions that encoded the results of trained neural networks. The latter work, in particular, proved enormously useful not only for this analysis but for all analyses, within $\mathrm{D} \varnothing$, that made use of neural networks.

It is impossible to use a standard methods to select such events with any efficiency. The $t \bar{t}$ analysis group was the first group at $\mathrm{D} \emptyset$ to use advanced multivariate methods.

\section{Future Interests}

The Tevatron has allowed the discovery of the top quark. However, the physics program at the Tevatron is by no means exhausted. There are still many things that we do not know about the top quark, and there is always the possibility to discover new things. With the accelerator improvements which are to be completed for Run II (due to begin in 2000), studies requiring large integrated luminosities including detailed top quark studies and searches for low mass Higgs particles will be carried out. The hardware improvements at both collider detectors will enable the upgraded Tevatron to be fully exploited. My plan is to participate in this effort while waiting for the next generation of accelerators to be built.

\section{Relevant Work Experience}

\section{Atmospheric \& Astrophysics Physics at Clemson}

As an undergraduate at Clemson University, I gained valuable research experience in atmospheric physics. It was during this period that I worked with Unix System $\mathrm{V}$ hardware and software. I used a Motorola workstation to study radar returns and to extract moments in the power spectrum of the returns. I used a PC to interface with a rain bucket for real time monitoring of rainfall levels. I maintained and used hardware and software for receiving real time satellite images from polar orbiters. I also assisted with the installation of software and hardware for a large wind profiling radar.

For my senior thesis, I worked on a computer simulation of stellar nuclear reactions. Such a simulation is quite complicated and requires knowledge of the nuclear reactions and state properties of the star as a function of radius. 


\section{Integration of Analysis with Neural Networks}

Due to the complicated nature of my research interests, I began to explore new ways of characterizing signal events. In top physics, where the signal and background are not significantly different and when the number of background events is orders of magnitude larger than the signal, it is necessary to seek new and innovative ways of finding signal events. It is often the case, in these situations, that modest gains in efficiency greatly improve the analysis.

The neural network is an approximation of the most efficient method possible for untangling signal from background. The neural network collapses an N-dimension space into a single dimension. The network properly accounts for correlations among import variables. The network output can be interpreted as the likelihood of the input event being a signal event. The network output can then be used to select signal events for further study and analysis.

I have worked with neural networks since March 1995. I have provided several analysis tools for users to integrate networks into their analysis, and I have assisted in porting the neural network code to other platforms.

\section{Real Time Systems}

During my first summer at the DØ experiment, I began to work with real time data acquisition systems for controls and monitoring for the D $\varnothing$ detector. I also started porting software to DØ computers and developed code for data acquisition using the EPICS controls and monitoring system. Indeed, I initiated the EPICS development at D $\varnothing$, under the guidance of my advisor. The D $\varnothing$ collaboration has formally adopted EPICS as their RUN II controls and monitoring toolkit.

During the 1997 D $\varnothing$ silicon detector fixed target test beam, I wrote real time code to format, verify and ship data from SVX IIe chips to an offline machine for storage and further analysis.

\section{Offline Support}

My most recent project was the re-processing of four terabytes of $D \varnothing$ data from Run 1b (1994-1996). This project was challenging as it required knowledge of a number of subsystems and the integration of these subsystems. Files were copied from a 30 terabyte mass storage system to system disk and three separate files were generated from the single input file. The system was required to be automated and self documenting. The project was completed in about three months.

\section{Upgrade}

For the D $\varnothing$ upgrade, I currently work to provide real time controls and monitoring system for all detector subgroups and assist with the online data acquisition system. I am also involved with the testing of SVX IIe chips that will be used in the DØ detector's silicon vertex detector. 


\section{Student Supervision \& Communication}

Thus far, my teaching experience has included one semester as a teaching assistant for two physics laboratory courses at Florida State University. During summers spent at DØ, I have advised and guided graduate students with both research and service projects. I enjoy giving physics talks and working with students.

I have presented a number of talks, regarding my work, to a variety of audiences. I feel that communication, formal or informal, is crucial for advancement and understanding in physics.

My long term career goal is to teach physics at a research-oriented university.

\section{References}

Professor Harrison Prosper, Department of Physics, Florida State University, Tallahassee, FL, 32306, (850)-644-6760, harry@hep.fsu.edu.

Professor Horst Wahl, Department of Physics, Florida State University, Tallahassee, FL, 32306, (850)-644-3509, wahl@hep.fsu.edu.

Dr. Pushpalatha C. Bhat, Fermi National Accelerator Laboratory, MS 357, P.O. Box 500, Batavia, IL 60510-0500, (630)840-8302, bhat@fnal.gov. 


\section{Publications in Refereed Journals}

1. "The DØ Detector at TeV33.", B.Abbott et al., D0note 3410, Fermilab-Pub98/124-E, hep-ex/9804011.

2. "Search for Heavy Pointlike Dirac Monopoles.", B.Abbott et al., Submitted to Phys. Rev. Letters, Fermilab-Pub-98/095-E, hep-ex/9803023.

3. "Limits on Anomalous $W W \gamma$ and $W W Z$ Couplings.", B.Abbott et al., Submitted to Phys. Rev. D, Rapid Communications, Fermilab-Pub-98/094-E, hep-ex/9803017.

4. "Search for Charge 1/3 Third Generation Leptoquarks in pbarp Collisions at $\sqrt{s}=1.8 \mathrm{TeV} . "$, B.Abbott et al., Submitted to Phys. Rev. Letters, Fermilab-Pub-98/081-E, hep-ex/9803009.

5. "Limits on $W W \gamma$ and $W W Z$ Couplings from $W$ Boson Pair Production.", B.Abbott et al., Submitted to Phys. Rev. D, Rapid Communications, Fermilab-Pub98/076-E, hep-ex/9803004.

6. "Measurement of the Shape of the Transverse Momentum Distribution of $W$ Bosons Produced in $\bar{p} p$ Collisions at $\sqrt{s}=1.8 \mathrm{TeV}$.", B.Abbott et al., Submitted to Phys. Rev. Letters, Fermilab-Pub-98/075-E, hep-ex/9803003.

7. "Search for the Decay $b \rightarrow X_{s} \mu^{+} \mu^{-}$.", B.Abbott et al., Accepted by Phys. Lett. B, Fermilab-Pub-98/033-E, hep-ex/9801027.

8. "Direct Measurement of the Top Quark Mass.", B.Abbott et al., Submitted to Phys. Rev. D, Fermilab-Pub-98/031-E, hep-ex/9801025.

9. "A Measurement of the $W$ Boson Mass.", B.Abbott et al., Submitted to Phys. Rev. D, Fermilab-Pub-97/422-E, hep-ex/9712029.

10. "A Measurement of the $W$ Boson Mass.", B.Abbott et al., Phys. Rev. Letters 80, 3000 (1998), Fermilab-Pub-97/423-E, hep-ex/9712028.

11. "Search for First Generation Scalar Leptoquark Pairs in $\bar{p} p$ Collisions at $\sqrt{s}=$ 1.8 TeV.", B.Abbott et al., Phys. Rev. Letters $\underline{80}, 2053$ (1998), Fermilab-Pub97/344-E, hep-ex/9710032.

12. " $Z \gamma$ Production in $\bar{p} p$ Collisions at $\sqrt{s}=1.8 \mathrm{TeV}$ and Limits on Anomalous $Z Z \gamma$ and $Z_{\gamma \gamma}$ Couplings.", B.Abbott et al., Accepted Phys. Rev. D, Fermilab-Pub97/363-E, hep-ex/9710031.

13. "Determination of the Mass of the $W$ boson Using the D $\varnothing$ detector at the Tevatron", B.Abbott et al., Submitted to Phys. Rev. D, Fermilab-Pub-97/328E, hep-ex/9710007. 
14. "Experimental Search for Chargino and Neutralino Production in Supersymmetry Models with a Light Gravitino.", B.Abbott et al., Phys. Rev. Letters 80, 442 (1998), Fermilab-Pub-97/273-E, hep-ex/9708005.

15. " Search for Scalar Leptoquark Pairs Decaying to Electrons and Jets in $\bar{p} p$ Collisions.", B.Abbott et al., Phys. Rev. Letters 79, 4321 (1997) , FermilabPub-97/252-E, hep-ex/9707033.

16. "Measurement of Dijet Angular Distributions and Search for Quark Compositeness.", B.Abbott et al.,Phys. Rev. Letters 으, 666 (1998), Fermilab-Pub97/237-E, hep-ex/9707016.

17. "Measurement of the Top Quark Mass Using Dilepton Events.", B.Abbott et al.,Phys. Rev. Letters $\underline{80}$, 2063 (1998), Fermilab-Pub-97/172-E, hepex/9706014.

18. " Color Coherent Radiation in Multijet Events from $\bar{p} p$ Collisions at $\sqrt{s}=1.8$ TeV.", B.Abbott et al., Phys. Lett. B $\underline{414}, 419$ (1997), Fermilab-Pub-97/201-E, hep-ex/9706012.

19. "Search for the Trilepton Signature from Associated Gaugino Pair Production.", B.Abbott et al., Phys. Rev. Letters 으, 1591 (1998), Fermilab-Pub97/153-E, hep-ex/9705015.

20. "Limits on $W W Z$ and $W W \gamma$ couplings from $\bar{p}-p \rightarrow e \nu j j X$ events at $\sqrt{s}=1.8$ TeV," B. Abbott et al.,Phys. Rev. Letters 79, 1441 (1997), Fermilab-Pub97/136-E, hep-ex/9705010.

21. "Measurement of the Top Quark Pair Production Cross Section in ppbar Collisions," S. Abachi et al., Phys. Rev. Letters 79, 1203 (1997), FermilabPub-97/109-E, hep-ex/9704015.

22. "Studies of Gauge Boson Pair Production and Trilinear Couplings", S. Abachi et al., Phys. Rev. D $\underline{56}, 6742$ (1997), Fermilab-Pub-97/088-E, hep-ex/9704004.

23. "Direct Measurement of the Top Quark Mass," S. Abachi et al., Phys. Rev. Letters $\underline{79}, 1197$ (1997), Fermilab-Pub-97/059-E, hep-ex/9703008.

24. "Study of the $Z Z \gamma$ and $Z \gamma \gamma$ Couplings in $Z(\rightarrow \nu \nu) \gamma$ Production," S. Abachi et al., Phys. Rev. Letters $\underline{78}, 3640$ (1997), Fermilab-Pub-97/047-E, hepex/9702011.

25. "Search for Top Squark Pair Production in the Dielectron Channel," S. Abachi et al., Accepted by Phys. Rev. D as a Brief Report, Fermilab-Pub-96/449-E.

26. "Search for Diphoton Events with Large Missing Transverse Energy in $\bar{p}-p$ Collisions at $\sqrt{s}=1.8 \mathrm{TeV}$," S. Abachi et al., Phys. Rev. Letters $\underline{78}, 2070$ (1997), Fermilab-Pub-96/446-E. 
27. "Search for a Fourth Generation -1/3 Quark via Flavor Changing Neutral Current Decay," S. Abachi et al., Phys. Rev. Letters 프, 3818 (1997), Fermilab-Pub-96/430-E.

\section{Internal Reports}

J. McDonald, "Nmake: A Routine to Build a Comis Function from a Trained Neural Network ," DØ Note 3157, February, 1997.

P. Bhat et al., "Measurement of top mass using lepton + jets events," DØ Note 3061, January, 1997.

S. Ahn et al., "Using Differences in the Widths of Jets in the Search for Top to All-Jets," DØ Note 3125, Nov, 1996.

S. Ahn et al., "Status Report: Search for Top in the All-Jets Channel," DØ Note 2807, December, 1995.

J. McDonald, "Porting and Installation of EPICS Control System on the D0SGI Clusters," DØ Note 2773, October, 1995. 